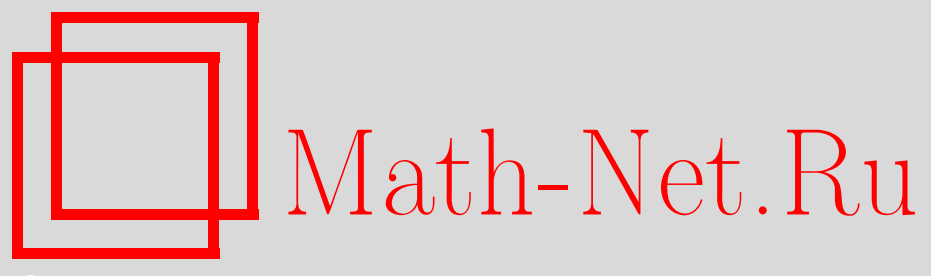

Ю. М. Полякова, Семейство категорий логтерминальных пар и автоморфизмы поверхностей, Изв. РАН. Сер. матем., 2010, том 74, выпуск 3, 103-156

DOI: https://doi.org/10.4213/im2687

Использование Общероссийского математического портала Math-Net.Ru подразумевает, что вы прочитали и согласны с пользовательским соглашением http://www . mathnet.ru/rus/agreement

Параметры загрузки:

IP : 54.210 .77 .194

26 апреля 2023 г., 14:13:24

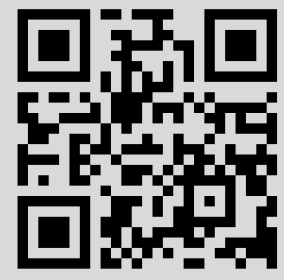


УДК 512.7

Ю. М. Полякова

\title{
Семейство категорий логтерминальных пар и автоморфизмы поверхностей
}

\begin{abstract}
На основе двумерной логтеории Мори развит категорный аппарат, позволяющий дать описание группы бирегулярных автоморфизмов комплексной квазипроективной поверхности с логтерминальными особенностями посредством групп бирегулярных автоморфизмов ее компактификаций.

Библиография: 13 наименований.
\end{abstract}

Ключевые слова: категории, бирегулярные автоморфизмы, бирациональные отображения, линки, логтерминальные особенности, правило Ope.

\section{§1. Введение}

Одна из основных проблем бирациональной геометрии состоит в факторизации бирациональных отображений алгебраических многообразий, т. е. в представлении этих отображений в виде композиции более простых. В настоящее время в качестве класса таких простых отображений обычно рассматривают класс линков, т. е. бирациональных отображений $\gamma$ таких, что $\gamma$ и $\gamma^{-1}$ стягивают не более одного неприводимого дивизора.

В случае решения задачи факторизации естественным образом возникает проблема явного описания полной системы соотношений в группоиде, порожденном множеством линков.

В [1] для комплексных расслоений Мори размерности два и три с терминальными $\mathbb{Q}$-факториальными особенностями доказана теорема о существовании разложения бирациональных отображений на элементарные линки. Основным методом доказательства является метод максимальных особенностей, опирающийся на неравенство Нётера-Фано. Случай расслоений Мори размерности два полностью изучен в статье [2], где дано полное описание всех линков и найдена полная система соотношений между последними.

Для пояснения возникающих проблем сформулируем теорию бирациональных отображений алгебраических многообразий в терминах теории категорий. Рассмотрим категорию $M$, объектами которой являются алгебраические многообразия, а морфизмами - бирациональные морфизмы. Очевидно, в этой категории множество всех морфизмов образует левый локализующий класс, т. е. выполнено левое правило Оре. Бирациональные отображения можно определить как классы эквивалентности диаграмм типа "верхний домик" (такие диаграммы обычно называются частичными разрешениями соответствующих им бирациональных отображений), для которых естественным образом с помощью

Работа выполнена при финансовой поддержке РФФИ (гранты № 07-01-00051, 99-01-01-204, 02-01-22005, 08-01-00-641-а) и CRDF (грант № RUM1-2661-MO-05).

(C) Ю. М. ПоляковА, 2010 
правила Оре определяется композиция. Таким образом, категория алгебраических многообразий с бирациональными отображениями изоморфна локализации категории $M$ по множеству всех ее морфизмов (весь категорный аппарат изложен в §2). Отметим, что в работе [1] разложение на элементарные линки по сути применялось не к классу эквивалентности "верхних домиков", а к некоторому его представителю, т.е. к частичному разрешению бирационального отображения.

Обобщением многообразий, рассмотренных в [1], могут служить двумерные и трехмерные логтерминальные пары, являющиеся расслоениями Мори. Их исследованию посвящена работа [3]. Двумерной логтерминальной парой $(V, C)$ называется пара такая, что $V$ - поверхность с $\mathbb{Q}$-факториальными особенностями, $C=\sum_{i=1}^{n} a_{i} C_{i}, a_{i} \in \mathbb{Q}, 0<a_{i} \leqslant 1$, - приведенная кривая на $V$, называемая границей, $C_{i}$ - неприводимые компоненты кривой $C$, причем существует бирациональный морфизм $\varphi: W \rightarrow V$, являющийся последовательным стягиванием $\left(K_{W}+C^{W}\right)$-экстремальных лучей дивизориального типа (см. $\left.\S 3\right)$ и такой, что $W$ - неособая поверхность, $C^{W}$ - кривая с нормальными пересечениями и $\varphi_{*}\left(C^{W}\right)=C$, где $\varphi_{*}$ - отображение прямого образа, а $K_{W}-$ канонический дивизор на $W$. Тогда естественным обобщением бирациональных морфизмов $\varphi: V^{\prime} \rightarrow V$ алгебраических многообразий являются морфизмы $\varphi:\left(V^{\prime}, C^{\prime}\right) \rightarrow(V, C)$, которые представляют собой такую последовательность стягиваний $\left(K_{V^{\prime}}+C^{\prime}\right)$-экстремальных лучей дивизориального типа, что $\varphi_{*}\left(C^{\prime}\right)=C$. Описанную таким образом категорию мы в дальнейшем будем называть категорией логтерминальных пар. Заметим, что морфизмы пар могут стягивать экстремальные лучи, не содержащиеся в границе, а такие морфизмы не сохраняют исходную открытую поверхность $U=V \backslash C$.

В работе [3] почти дословно воспроизводится техника Корти для логтерминальных пар, т. е. с помощью метода максимальных особенностей осуществляется разложение диаграмм типа "верхний домик" на элементарные "верхние домики", называемые, как и раньше, линками. Однако для категории логтерминальных пар это не приводит к таким сильным результатам, как в [1] и [2], так как в общем случае для "верхних домиков" не определена композиция, что, во-первых, не позволяет строить “большие домики" из элементарных линков и, во-вторых, лишает осмысленности постановку задачи о нахождении множества определяющих соотношений.

Отсутствие естественной композиции “верхних домиков" объясняется невыполненностью правила Оре в категории логтерминальных пар в связи с двумя основными причинами. Первая из них (устранимая) состоит в том, что коэффициенты $a_{i}$ при кривых $C_{i}$ могут быть различны. Вторая (не устранимая при использовании техники из [1], т. е. при рассмотрении расслоения Мори) состоит в том, что можно стягивать экстремальные лучи, не принадлежащие границе.

Описанные выше препятствия к выполнению правила Оре в категории логтерминальных пар привели нас к рассмотрению семейства категорий $R_{p}$, где $p \in \mathbb{Q}, 0 \leqslant p \leqslant 1$, эквивалентным таким подкатегориям в категории логтерминальных пар, в которых коэффициенты $a_{i}$ при кривых $C_{i}$ совпадают и равны фиксированному значению $p$ и все стягивания происходят внутри границы. В этих категориях правило Оре выполнено при $p<1$ (см. §4). Рассматривать их необходимо в связи с тем, что в категории $R_{1}$ (именно ее в результате мы будем исследовать) правило Оре не выполнено, а выполнено только на некоторой ее подкатегории, описываемой посредством перехода к пределу при $p \rightarrow 1$. 
Изначальной целью настоящего исследования было применение логтеории Мори к описанию группы бирегулярных автоморфизмов комплексной квазипроективной поверхности посредством групп автоморфизмов ее компактификаций. Эта задача решалась в серии работ М.Х. Гизатуллиным и В.И. Даниловым [4]-[9]. Авторы изучали неособые поверхности и компактификации, ввиду чего их доказательства были очень громоздки. Рассмотрение особых, а точнее логтерминальных, поверхностей и компактификаций позволяет существенно упростить решение задачи.

Упомянутая выше задача об описании группы автоморфизмов квазипроективной поверхности сводится к разложению морфизмов категории частных рассматриваемых нами категорий $R_{p}$ на элементарные линки и к описанию полной системы соотношений между последними (см. §8). Подход, основанный на двумерной логтеории Мори, т. е. на рассмотрении не просто бирациональных отображений, а отображений пар (поверхность и кривая на ней), является наиболее естественным для решения поставленной задачи, поскольку мы рассматриваем компактификации неполной поверхности. При этом очевидно, что стягивания экстремальных лучей, не лежащих в границе, не являются допустимыми морфизмами в нашем случае, так как не сохраняют исходную квазипроективную поверхность (что соответствует определению категорий $R_{p}$ ). Решению задачи факторизации для данного случая, корректному определению композиции элементарных линков и поиску полной системы соотношений между последними посвящена настоящая работа. В конце статьи мы показываем, как на основе этих данных описать группы автоморфизмов некоторого класса квазипроективных поверхностей.

Ограничившись рассмотрением подкатегорий категории логтерминальных пар, для которых коэффициенты $a_{i}$ при кривых $C_{i}$ совпадают и равны фиксированным значениям $p$ (такие категории содержат подкатегории, эквивалентные вводимым нами категориям $R_{p}$ ), мы могли бы воспользоваться разложением на линки для расслоений Мори в таких подкатегориях с помощью [3] и получить доминирующее разложение на линки в категории "верхних домиков" над $R_{p}$ (очевидно, что поверхности, соответствующие парам, являющимся объектами категорий $R_{p}$, могут иметь в общем случае большой ранг группы Пикара и не являться расслоениями Мори). Далее, используя выполненность правила Оре для категорий $R_{p}$, можно было бы дать описание полной системы соотношений между линками и в случае отсутствия нетривиальных соотношений решить задачу об автоморфизмах. Однако подобное решение было бы не менее громоздко, чем конструкция Гизатуллина и Данилова. Поэтому мы нигде не опираемся на конструкцию Бруно и Мацуки и приводим совершенно другой подход к решению задачи (см. §8).

Основным практическим результатом статьи является построение категорного аппарата, значительно упрощающего предложенный ранее в [9] подход к описанию группы бирегулярных автоморфизмов комплексной квазипроективной поверхности. Поэтому читателю, интересующемуся конкретными приложениями, мы советуем сразу перейти к последним двум параграфам, а потом просмотреть предыдущий текст. Основным теоретическим результатом работы мы считаем исследование возможности введения композиции для отображений логтерминальных пар, которое позволяет нам дать описание полной системы соотношений между линками. Отметим, что без описания соотношений (а точнее, без доказательства отсутствия нетривиальных соотношений) поставленная 
задача не может быть решена. Мы надеемся, что теоретические (категорные) исследования, проведенные в работе, проясняют логтеорию Мори и могут быть полезны при рассмотрении аналогичных задач в больших размерностях.

Изложим теперь более подробно содержание настоящей работы.

В $\S 2$ мы развиваем категорный аппарат, необходимый нам в дальнейшем. По произвольной малой категории $T$, ее подкатегории и подмножеству в множестве объектов строятся нужные нам категории диаграмм (например, категории “стрелок" и "верхних домиков"). Приводятся определения левого и правого правил Оре. Показано, что если в категории $T$ выполнено левое правило Оре, то в категории “верхних домиков" над этой категорией можно ввести отношение эквивалентности, частично определить композицию классов эквивалентности "верхних домиков" и, таким образом, построить категорию частных $\widetilde{T}$ для данной категории $T$ (см. определение 2.7). Мы даем определение лево- и правожестких объектов категории, а также $\mathbb{Z}_{\geqslant 0}$-упорядоченных категорий. Для

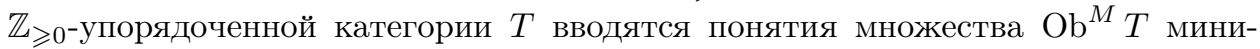
мальных объектов $T$, множества $M_{T}(\Theta)$ минимизаций произвольного подмножества $\Theta$ объектов категории $T$, а также множества минимальных разрешений объекта в подкатегории $\Omega$ категории $T$ и множества минимальных разрешений морфизма $\gamma$ категории частных $\widetilde{T}$. Заметим, что правожесткость минимального объекта $\mathbb{Z}_{\geqslant 0}$-упорядоченной категории означает отсутствие нетривиальных "верхних домиков" над минимальными объектами, концом одной из "стрелок" которых является этот объект; в случае выполненности в категории левого правила Оре правожесткость минимального объекта означает отсутствие морфизмов категории частных из данного объекта в любой другой минимальный объект. Нами доказана теорема 2.16 о том, что в $\mathbb{Z}_{\geqslant 0}$-упорядоченной категории выполнено правое правило Оре тогда и только тогда, когда любой ее объект имеет единственную с точностью до изоморфизма минимизацию. В конце $\S 2$ для $\mathbb{Z}_{\geqslant 0}$-упорядоченных категорий даются определения разложимости морфизмов, а также замыкания одного подмножества объектов в другом.

В $\S 3$ мы сначала напоминаем некоторые понятия и теоремы из теории Мори. Затем даем определение $\mathbb{Z}_{\geqslant 0}$-упорядоченной разложимой категории пар $R$, объектами которой являются пары $(V, C)$ такие, что $V$ - нормальная проективная поверхность с $\mathbb{Q}$-факториальными особенностями, $C$ - приведенная кривая на $V$, а морфизмами $\varphi:(V, C) \rightarrow\left(V^{\prime}, C^{\prime}\right)$ являются бирациональные морфизмы $\varphi: V \rightarrow V^{\prime}$ такие, что $C^{\prime}=\varphi^{-1}(C)$, т. е. $\varphi$ стягивает компоненты кривой $C$. Структура $\mathbb{Z}_{\geqslant 0}$-упорядоченной категории естественным образом определяется на $R$ числом Пикара. В категории $R$ мы вводим семейство $\mathbb{Z}_{\geqslant 0}$-упорядоченных разложимых подкатегорий $R_{p} p$-логтерминальных пар, где $p \in \mathbb{Q}, 0 \leqslant p \leqslant 1$. Объектами этих подкатегорий являются пары $(V, C)$ такие, что пара $(V, p C)$ логтерминальна, а морфизмами - последовательности стягиваний $\left(K_{V}+p C\right)$-экстремальных лучей дивизориального типа, лежащих в $C$, где $K_{V}$ - канонический дивизор на $V$. Мы также определяем левые и правые предельные категории при стремлении параметра к определенному значению. Далее исследуются элементарные свойства перечисленных выше категорий, в частности доказываются теорема 3.14 об инвариантном представлении морфизмов и разложимость предельных категорий.

В $\S 4$ мы определяем категорию неособых пар $R^{N}$ как полную подкатегорию в категории $R$, множество объектов которой состоит из пар $(V, C)$ таких, что 
$V$ - неособая поверхность, а $C$ - кривая с простыми нормальными пересечениями (это именно та категория, которую рассматривали Гизатуллин и Данилов). Далее исследуются общие свойства категорий $R, R^{N}$ и $R_{p}$. В теореме 4.6 нами доказана выполненность левого правила Оре в категории $X$, где $X$ - одна из категорий $R, R^{N}, R_{p}$ при $p<1$ или предельная категория. Отсюда следует, что хорошо определена категория частных $\widetilde{X}$ категории $X$. В утверждении 4.7 мы доказываем, что любой объект категории $X$ имеет единственное минимальное разрешение особенностей с точностью до изоморфизма и что любой морфизм категории частных $\widetilde{X}$ имеет единственное минимальное разрешение с точностью до изоморфизма. В теореме 4.12 и замечании 4.13 установлено, что категории $X$ и $\widetilde{X}$ являются полными подкатегориями в $R$ и $\widetilde{R}$ соответственно. Мы также показываем, что правило Оре не выполнено в категории $R_{1}$ и что $R_{1}$ не является полной подкатегорией в категории $R$.

В $\S 5$ мы изучаем более тонкие свойства категорий $R_{p}$. В теореме 5.12 произведена классификация особенностей объектов этих категорий и элементарных морфизмов, соответствующих стягиваемым в данных категориях экстремальным лучам. Из этой классификации вытекают следующие результаты (см. следствие 5.13):

1 ) категория $R_{p_{1}}$ является полной подкатегорией в категории $R_{p_{2}}$ при $4 / 5<$ $p_{1}<p_{2}<1$, и обе они являются полными подкатегориями в категории $R_{1_{-}}$; категория $R_{p_{-}} \sim R_{p}$ является полной подкатегорией в категории $R_{p_{+}}$при $4 / 5<p<1$, где $R_{p_{-}}$и $R_{p_{+}}$- соответственно левые и правые предельные категории при стремлении параметра к $p$; аналогичные утверждения выполнены для категорий частных;

2 ) категория $R_{p_{1}}$ является полной подкатегорией в категории $R_{p_{2}}$ при $0<$ $p_{2}<p_{1}<1 / 3$, и обе они являются полными подкатегориями $R_{1_{-}}$; категория $R_{p_{+}} \sim R_{p}$ является полной подкатегорией в категории $R_{p_{-}}$при $0<p<1 / 3$; аналогичные утверждения выполнены для категорий частных;

3) $\mathrm{Ob} R_{1_{-}}=\mathrm{Ob} R_{1}$.

В $\S 6$ мы определяем функтор разрешения особенностей $\mathcal{N}_{X}: X \rightarrow R^{N}$ и индуцируемый им функтор категорий частных $\widetilde{\mathcal{N}}_{X}: \widetilde{X} \rightarrow \widetilde{R}^{N}$, где $X-$ одна из категорий $R_{p}$ при $p<1$ или предельная категория. В теореме 6.2 мы доказываем, что при $4 / 5<p<1$ любой минимальный объект категории $R^{N}$ имеет единственную с точностью до изоморфизма минимизацию в категории $R_{p}$, т. е. морфизм на минимальный в $R_{p}$ объект. Заметим, что при $p<4 / 5$ минимизация может быть не единственна. Поэтому можно определить функтор минимизации $\widetilde{\mathcal{M}}_{R_{p}}: \widetilde{R}^{N M} \rightarrow \widetilde{R}_{p}^{M}$, где $\widetilde{R}^{N M}$ и $\widetilde{R}_{p}^{M}-$ полные подкатегории в $\widetilde{R}^{N}$ и $\widetilde{R}_{p}$, множества объектов которых являются множествами минимальных объектов соответствующих категорий. Функтор минимизации является квазиобратным к ограничению функтора разрешения особенностей на соответствующие подкатегории. Отметим, что не для любого минимального объекта категории $R_{p}$ его разрешение особенностей будет минимальным объектом категории $R^{N}$. Нами доказана следующая важная для дальнейших построений теорема 6.13 о замкнутости: пусть $(V, C),\left(V^{\prime}, C^{\prime}\right) \in \mathrm{Ob} \widetilde{R}_{p}^{M}$ при $4 / 5<p<1$ и их разрешения особенностей минимальны в $R^{N}$, пусть, далее, $\gamma:(V, C)-\rightarrow\left(V^{\prime}, C^{\prime}\right), \gamma \in \operatorname{Mor} \widetilde{R}_{p}^{M}$ (пунктирными стрелками мы обозначаем морфизмы категорий частных) и пара морфизмов $\varphi:\left(W, C^{W}\right) \rightarrow(V, C), \varphi^{\prime}:\left(W, C^{W}\right) \rightarrow\left(V^{\prime}, C^{\prime}\right)$ - минимальное разрешение $\gamma$; тогда для любого $\varphi^{\prime \prime}:\left(W, C^{W}\right) \rightarrow\left(V^{\prime \prime}, C^{\prime \prime}\right)$ такого, что $\varphi \in$ Mor $R_{p}$, 
$\left(V^{\prime \prime}, C^{\prime \prime}\right) \in \mathrm{Ob} \widetilde{R}_{p}^{M}$, разрешение особенностей пары $\left(V^{\prime \prime}, C^{\prime \prime}\right)$ также будет минимальным объектом категории $R^{N}$.

В $\S 7$ для пар $(V, C)$, являющихся объектами категории $X$, где $X$ - одна из категорий $R^{N}, R_{p}$ или предельная категория, по аналогии с [5] мы определяем эффективную кривую $F_{X}(V, C) \subset C$ как кривую, состоящую из всех неприводимых компонент $F_{i}$ кривой $C$, для которых существуют морфизмы $\varphi:\left(W, C^{W}\right) \rightarrow(V, C), \varphi_{i}:\left(W, C^{W}\right) \rightarrow\left(V^{i}, C^{i}\right)$ такие, что $\varphi_{i}$ стягивает $\varphi_{\text {соб }}^{-1}\left(F_{i}\right)$, где $\varphi_{\text {соб }}^{-1}\left(F_{i}\right)$ - собственный прообраз кривой $F_{i}$. Эта кривая в случае выполнения в $X$ левого правила Оре инвариантна относительно морфизмов категории частных, где инвариантность понимается в следующем смысле. Пусть пара морфизмов $\varphi:\left(W, C^{W}\right) \rightarrow(V, C), \varphi^{\prime}:\left(W, C^{W}\right) \rightarrow\left(V^{\prime}, C^{\prime}\right)$ - разрешение морфизма $\gamma:(V, C) \rightarrow\left(V^{\prime}, C^{\prime}\right)$; тогда

$$
F_{X}\left(V^{\prime}, C^{\prime}\right)=\varphi_{*}^{\prime}\left(\varphi^{-1}\left(F_{X}(V, C)\right)\right)
$$

(Здесь $\varphi^{-1}$ - отображение полного прообраза.) Очевидно, что при $F_{X}(V, C)=0$ пара $(V, C)$ является правожестким объектом категории $X$.

В утверждении 7.3 мы доказываем, что если $(V, C),\left(V^{\prime}, C^{\prime}\right) \in \mathrm{Ob}^{M} X$, где через $\mathrm{Ob}^{M} X$ обозначается множество минимальных объектов категории $X$, $\varphi:\left(W, C^{W}\right) \rightarrow(V, C), \varphi^{\prime}:\left(W, C^{W}\right) \rightarrow\left(V^{\prime}, C^{\prime}\right)$ и не существует морфизмов $\rho, \psi, \psi^{\prime} \in \operatorname{Mor} X$ таких, что $\varphi=\psi \circ \rho, \varphi^{\prime}=\psi^{\prime} \circ \rho$, то точки неопределенности отображения $\varphi^{-1}$ лежат в $F_{X}(V, C)$, а точки неопределенности отображения $\left(\varphi^{\prime}\right)^{-1}$ лежат в $F_{X}\left(V^{\prime}, C^{\prime}\right)$.

В теоремах $7.19,7.20$ и 7.22 произведена классификация таких кривых и получен конструктивный способ их нахождения (последнее не было сделано в [5] для категории $R^{N}$ ). Установлено, что любая связная компонента кривой $F_{X}(V, C)$ при $(V, C) \in \mathrm{Ob}^{M} X$ содержит неприводимую компоненту с неотрицательным индексом самопересечения. Доказано, что для $(V, C) \in \mathrm{Ob}^{M}(X)$ таких, что $\mathcal{N}_{X}(V, C) \in \mathrm{Ob}^{M} R^{N}$, кривая $F_{X}(V, C)$ в случае ее непустоты имеет один из трех описанных ниже типов, т. е. является:

1) связной кривой арифметического рода нуль с нормальными пересечениями такой, что любая ее неприводимая компонента пересекается с не более чем двумя другими неприводимыми компонентами, и если неприводимая компонента пересекается с двумя компонентами, то она не проходит через особенности поверхности $V$, если неприводимая компонента пересекается с одной компонентой, то она содержит не более одной особой точки $V$ типа $A_{n}$ (ввиду того, что мы рассматриваем особенности пар, а не поверхностей, особая точка поверхности $V$ типа $A_{n}$, лежащая на кривой $C$, в наших обозначениях имеет тип $0_{m, n, k}$, где $k \leqslant 2, m-$ кратность этой точки на кривой $C$; см. определение $5.2,2)$ ), а если кривая $F_{X}(V, C)$ неприводима, то она проходит через не более чем две особых точки $V$ типа $A_{n}$;

2) объединением неприводимых непересекающихся рациональных кривых с нулевым индексом самопересечения;

3) колесом, т. е. связной не проходящей через особенности $V$ кривой арифметического рода один с нормальными пересечениями, каждая неприводимая компонента которой рациональна, $F_{X}(V, C)\left(C-F_{X}(V, C)\right)=0$ (см. определение 5.4).

Мы также доказываем, что $F_{X}(V, C)$ совпадает с максимальной кривой, содержащейся в $C$ и имеющей один из трех описанных выше типов. 
В теореме 7.22 установлена инвариантность $F_{X}(V, C)$ относительно функтора разрешения особенностей при $X \neq R_{1}$ (в $R_{1}$ и $R_{p}$ при $p<4 / 5$ эти утверждения не верны), поэтому классификация $F_{X}(V, C)$ в данном случае сводится к их классификации в $R^{N}$, полученной в [5]. Для доказательства инвариантности $F_{X}(V, C)$ относительно данного функтора оказывается необходимым ввести некоторое множество "элементарных" морфизмов категории $\widetilde{R}^{N M}$, практически совпадающих с определенными в [9]. Эти морфизмы также используются для приведения пары к стандартному виду (наше определение стандартности несколько шире, чем используемое у Гизатуллина и Данилова). С помощью приведения пары к стандартному виду мы устанавливаем, что любая (за одним исключением) квазипроективная поверхность, имеющая компактификацию $(V, C)$ такую, что кривая $F_{X}(V, C)$ имеет тип 1 , может быть компактифицирована так, что кривая $F_{X}(V, C)$ будет неприводимой кривой типа 1 . Исключением является тот случай, когда поверхность имеет такую компактификацию, что $F_{X}(V, C)$ состоит из двух компонент $F_{1}, F_{2}$, не проходящих через особенности $V$ и таких, что $F_{1}^{2}=F_{2}^{2}=1,\left(F_{1}, F_{2}\right)=1$.

Опишем классификацию кривых $F_{R_{1}}(V, C)$ для различных пар. Пусть $(V, C) \in \mathrm{Ob}^{M} R_{1_{-}}$и $\mathcal{N}_{R_{1_{-}}}(V, C) \in \mathrm{Ob}^{M} R^{N}$. Тогда

$$
F_{X}(V, C)\left(C-F_{X}(V, C)\right)=0,
$$

любая связная компонента $F=F_{R_{1}}(V, C)$ неприводима, неособа и либо $F$ - объединение непересекающихся кривых с нулевым индексом самопересечения, не проходящих через особенности $V$, либо $F$ состоит из одной компоненты, проходящей через не более чем две особые точки поверхности $V$ типа $A_{n}$. Последний случай в точности соответствует нашим стандартным парам.

В $\S 8$ мы определяем категорию $\widetilde{R}_{1}^{M}$ и строим разложение морфизмов категории $\widetilde{R}_{1}^{M}$ на элементарные. Нам понадобится приводимая ниже конструкция.

Под графом мы будем понимать неориентированный граф в обычном смысле, т. е. задано множество вершин, множество ребер, и каждому ребру сопоставлены две вершины, являющиеся его концами. Подграфы графа определяются естественным образом. Пусть $\mathcal{G}$ - граф и $\mathcal{G}^{\prime}-$ подграф в $\mathcal{G}$; тогда определим дополнение графа $\mathcal{G}^{\prime}$ в $\mathcal{G}$ как граф $\mathcal{G} \backslash \mathcal{G}^{\prime}$, полученный из $\mathcal{G}$ удалением всех вершин и ребер $\mathcal{G}^{\prime}$, а также ребер, соединяющих вершины $\mathcal{G}$ с вершинами $\mathcal{G}^{\prime}$. Пусть теперь $\mathcal{G}$ - дерево (т. е. связный граф без циклов) и $\mathcal{G}^{\prime}$ - подграф в $\mathcal{G}$; тогда $\mathcal{G}^{\prime}$ мы будем называть внешним, если $\mathcal{G} \backslash \mathcal{G}^{\prime}$ - дерево. Определим замыкание подграфа $\mathcal{G}^{\prime}$ в $\mathcal{G}$ как граф $\overline{\left(\mathcal{G}^{\prime}\right)^{\mathcal{G}}}$, являющийся минимальным поддеревом в $\mathcal{G}$, содержащим $\mathcal{G}^{\prime}$. Аналогично определим замыкание подграфа в объединении деревьев.

Пусть $D$ - приведенная кривая с нормальными пересечениями на некоторой поверхности. Тогда по этой кривой мы можем построить ассоциированный с ней граф $\mathcal{G}(D)$ следующим образом: каждой неприводимой компоненте кривой $D$ сопоставим вершину графа $\mathcal{G}(D)$, а каждой точке пересечения компонент сопоставим ребро, соединяющее вершины, соответствующие пересекающимся компонентам. Отметим, что граф $\mathcal{G}(D)$ может иметь петли и кратные ребра.

Нами доказана следующая теорема: пусть $(V, C) \in \mathrm{Ob} R_{1_{-}}, F \subset H \subset$ $G \subset C$ - кривые на $V,(G, C-G)=0$, граф $\mathcal{G}(G)$ является деревом, кривая $H$ стягиваема в категории $R_{1_{-}}$и $\mathcal{G}(F)$ - внешний подграф в $\mathcal{G}(G)$; тогда 
кривая $F$ стягиваема в категории $R_{1}$. Эта теорема имеет очень важные следствия, а именно:

1) если $(V, C) \in \mathrm{Ob}^{M} R_{1_{-}}$и кривая $F_{R_{1}}(V, C)$ непуста, то $F_{R_{1}}(V, C)=$ $F_{R_{1}}(V, C)$;

2 ) можно определить полную подкатегорию $\widetilde{R}_{1}^{M}$ в категории $\widetilde{R}_{1-}^{M}$, объектами которой являются пары $(V, C)$ такие, что $F_{R_{1}}(V, C) \neq 0$;

$3)$ если $\gamma \in \operatorname{Mor} \widetilde{R}_{1_{-}}^{M}$, пара морфизмов $\varphi:\left(W, C^{W}\right) \rightarrow(V, C), \varphi^{\prime}:\left(W, C^{W}\right) \rightarrow$ $\left(V^{\prime}, C^{\prime}\right)$ - минимальное разрешение $\gamma, F=F_{R_{1_{-}}}(V, C), F^{\prime}=F_{R_{1_{-}}}\left(V^{\prime}, C^{\prime}\right), F^{W}=$ $F_{R_{1_{-}}}\left(W, C^{W}\right)$ и $\mathcal{G}(F)$ - объединение конечного числа деревьев, то

$$
\mathcal{G}\left(F^{W}\right)=\mathcal{G}\left(F_{R_{1_{-}}}\left(W, C^{W}\right)\right)=\mathcal{G}\left(\varphi^{-1}(F)\right)=\overline{\left(\mathcal{G}\left(\varphi_{\text {соб }}^{-1}(F)\right)+\mathcal{G}\left(\left(\varphi^{\prime}\right)_{\text {соб }}^{-1}\left(F^{\prime}\right)\right)\right)^{\mathcal{G}\left(C^{W}\right)}}
$$

Очевидно что, если $(V, C),\left(V^{\prime}, C^{\prime}\right) \in \mathrm{Ob} \widetilde{R}_{1}^{M}$, то граф кривой $F^{W}$ является объединением цепей $F_{i}^{W}$, первой вершине каждой из которых соответствует собственный прообраз соответствующей связной компоненты кривой $F$, а последней - собственный прообраз компоненты кривой $F^{\prime}$. Исходя из этого, мы немедленно получаем разложение морфизма $\gamma$ на элементарные морфизмы $\gamma_{i}^{j}$, где $1 \leqslant i \leqslant n, 1 \leqslant j \leqslant m_{i}-1, n$ - число связных компонент $F_{i}^{W}$ кривой $F^{W}$, а $m_{i}$ - количество вершин $i$-й компоненты. Граф кривой $F^{W_{i}^{j}}$ на минимальном разрешении $\left(\left(W_{i}^{j}, C^{W_{i}^{j}}\right) \varphi_{i}^{j}, \varphi_{i}^{j+1}\right)$ морфизма $\gamma_{i}^{j}$ представляет собой $n-1$ изолированных вершин и две вершины, соединенные отрезком. Эти две вершины являются образами $j$-й и $(j+1)$-й вершин $i$-й связной компоненты кривой $F^{W}$.

Легко доказывается (см. теорему 8.11), что между морфизмами, соответствующими одной связной компоненте, отсутствуют соотношения, а морфизмы, относящиеся к разным компонентам, коммутируют.

Если минимальные разрешения особенностей пар $(V, C)$ и $\left(V^{\prime}, C^{\prime}\right)$ являются минимальными объектами категории $R^{N}$, то из теоремы о замкнутости следует, что таковыми же будут и все промежуточные объекты разложения. Применив функтор разрешения особенностей к данному разложению, мы получим, что построенным нами элементарным морфизмам соответствуют элементарные морфизмы категории $\widetilde{R}^{N M}$, упомянутые выше.

В $\S 9$ мы описываем группы автоморфизмов квазипроективных поверхностей. Мы рассматриваем квазипроективные поверхности $U$ с 1-логтерминальными особенностями, т. е. $U$ имеет $\mathbb{Q}$-факториальные особенности, и существует бирациональный морфизм $\varphi: W \rightarrow V$, являющийся последовательным стягиванием $\left(K_{W}+E_{W}\right)$-экстремальных лучей дивизориального типа $E_{i}($ см. $\S 3)$ и такой, что $W$ неособа, $E^{W}=\sum_{i=1}^{n} E_{i}$ - компактная кривая с нормальными пересечениями и $\varphi_{*} E^{W}=0$.

Далее мы определяем категорию $T^{U}$ компактификаций поверхности $U$ в категории $R_{1}$ как категорию, объектами которой являются тройки $(\rho, V, C)$, где $\rho: U \hookrightarrow V$ - открытое вложение, $(V, C) \in \mathrm{Ob} R_{1}=\mathrm{Ob} R_{1_{-}}$и $C=V \backslash \rho(U)$, а морфизмами $\varphi:(\rho, V, C) \rightarrow\left(\rho^{\prime}, V^{\prime}, C^{\prime}\right)$ - морфизмы $\varphi:(V, C) \rightarrow\left(V^{\prime}, C^{\prime}\right)$ такие, что $\varphi \in$ Mor $R_{1_{-}}$и $\varphi \circ \rho=\rho^{\prime}$.

Через $R_{1_{-}}^{U}$ мы обозначаем полную подкатегорию в $R_{1_{-}}$, объектами которой являются пары, компактифицирующие поверхность $U$ в этой категории. Категория $R_{1_{-}}^{U}$ получается из категории $T^{U}$ применением функтора забывания открытого вложения $\rho$. 
Выполнение правила Оре в категории $R_{1_{-}}$индуцирует его выполнение в категории $T^{U}$, поэтому корректно определена категория частных $\widetilde{T}^{U}$ категории $T^{U}$. Через $\widetilde{R}_{1_{-}}^{U}$ обозначается категория частных категории $R_{1-}^{U}$.

Если поверхность $U$ имеет 1-логтерминальные особенности и $\operatorname{Aut}(U)-$ группа ее бирегулярных автоморфизмов, то существует компактификация $(\rho, V, C) \in \mathrm{Ob}^{U}$. Поскольку категория $R_{1_{-}}$является полной подкатегорией в категории $R$, любому бирегулярному автоморфизму $g \in \operatorname{Aut}(U)$, заданному вместе с компактификациями $(\rho, V, C),\left(\rho^{\prime}, V^{\prime}, C^{\prime}\right) \in \mathrm{Ob} T^{U}$, взаимно однозначно соответствует $\gamma \in \operatorname{Mor} \widetilde{R}_{1_{-}}^{U}, \gamma=\rho^{\prime} \circ g \circ \rho^{-1}:(V, C)-\rightarrow\left(V^{\prime}, C^{\prime}\right)$.

Для поверхности $U$, имеющей 1-логтерминальные особенности, рассматриваются такие $(\rho, V, C) \in \mathrm{Ob} T^{U}$, что $(V, C) \in \mathrm{Ob}^{M} R_{1_{-}}$(очевидно, что такие компактификации всегда существуют). В случае, когда кривая $F_{R_{1}}(V, C)$ может быть приведена к стандартному виду, рассматриваются только стандартные компактификации. Обозначим через $T^{U, M}$ полную подкатегорию в $T^{U}$, соответствующую определенному выше классу компактификаций.

Чтобы не вдаваться в излишние подробности, мы ограничились рассмотрением таких поверхностей $U$, для компактификаций которых выполнено следующее условие: $F_{R_{1_{-}}}(V, C)\left(C-F_{R_{1_{-}}}\right)=0$. (Легко видеть, что общий случай может быть сведен к данному частному.) Тогда согласно результатам из $\S 7$ имеются следующие альтернативы:

1) $F_{R_{1}}(V, C) \neq 0$; тогда $F_{R_{1}}(V, C)$ либо является объединением непересекающихся кривых с нулевым индексом самопересечения, не проходящих через особенности $V$, либо состоит из одной компоненты, проходящей через не более чем две особые точки поверхности $V$ типа $A_{n}$;

2) $F_{R_{1}}(V, C)=0$, а $F_{R_{1_{-}}}(V, C) \neq 0$; тогда либо $F_{R_{1_{-}}}(V, C)$ является колесом, либо $U$ имеет компактификацию такую, что $F_{R_{1}}(V, C)$ состоит из двух компонент $F_{1}, F_{2}$, не проходящих через особенности $V$ и таких, что $F_{1}^{2}=F_{2}^{2}=1$, $\left(F_{1}, F_{2}\right)=1$;

3) $F_{R_{1_{-}}}(V, C)=0$.

Сначала рассматривается случай $F_{R_{1}}(V, C) \neq 0$. В $\S 8$ определены элементарные морфизмы категории $\widetilde{R}_{1}^{M}$, на которые можно разложить любой морфизм этой категории. Очевидно, любой морфизм категории $\widetilde{T}^{U, M}$ также раскладывается на соответствующие этим морфизмам элементарные морфизмы. Тогда по аналогии с [9] определяется граф компактификаций $\Delta_{U}$ как граф, вершинами которого являются классы изоморфных компактификаций поверхности $U$, а ребрами - элементарные морфизмы. Такое определение корректно ввиду того, что элементарные морфизмы переводят изоморфные компактификации в изоморфные. Доказательство существования разложения морфизмов категории $\widetilde{T}^{U, M}$ на элементарные равносильно доказательству связности графа $\Delta_{U}$. Условием $g(\rho)=g \circ \rho$ определяется действие группы $\operatorname{Aut}(U)$ на этом графе. Легко проверяется, что факторграф графа $\Delta_{U}$ по данному действию изоморфен графу пар, компактифицирующих поверхность $U$ в категории $R_{1}^{M}$, вершинами которого являются классы изоморфных пар, а ребрами - элементарные морфизмы категории $\widetilde{R}_{1}^{M}$. Далее по этому факторграфу и группе $\operatorname{Aut}(U)$ согласно классической конструкции Серра [10] строится граф групп $\Gamma_{U}$ и рассматривается его фундаментальная группа. Подробное описание этой конструкции приведено в настоящей работе. 
Если $F_{R_{1}}(V, C)$ состоит из одной неприводимой компоненты, то согласно результатам из $\S 8$ между элементарными морфизмами отсутствуют нетривиальные соотношения. Поэтому граф $\Delta_{U}$ не имеет циклов и, следовательно, является деревом. Тогда согласно теореме Серра (см. [10, теорема 13]) группа $\operatorname{Aut}(U)$ изоморфна фундаментальной группе упомянутого выше графа групп $\Gamma_{U}$. Таким образом, получено полное описание группы $\operatorname{Aut}(U)$ для данного случая.

Если $F_{R_{1}}(V, C)$ состоит из нескольких неприводимых компонент, то элементарные морфизмы, соответствующие различным неприводимым компонентам, коммутируют. Поэтому граф $\Delta_{U}$ имеет циклы и не является деревом, ввиду чего согласно результатам из [10] группа $\operatorname{Aut}(U)$ изоморфна фактору фундаментальной группы графа $\Gamma_{U}$ по фундаментальной группе графа $\Delta_{U}$, порожденной соотношениями коммутации.

Далее рассматривается случай, когда $F_{R_{1}}(V, C)=0$, а $F_{R_{1_{-}}}(V, C) \neq 0$. Если $F_{R_{1}}(V, C)$ является колесом, нами не получено разложение морфизмов категории $\widetilde{R}_{1_{-}, N}^{M}$ на элементарные, и вряд ли оно могло бы оказаться полезным для описания группы $\operatorname{Aut}(U)$, хотя легко доказать, что связная компонента единицы этой группы совпадает со связной компонентой единицы группы бирегулярных автоморфизмов любой компактифицирующей ее пары.

В случае, когда $U$ имеет компактификацию такую, что $F_{R_{1}}(V, C)$ состоит из двух компонент $F_{1}, F_{2}$, не проходящих через особенности $\bar{V}$ и таких, что $F_{1}^{2}=F_{2}^{2}=1,\left(F_{1}, F_{2}\right)=1$, разложение на линки легко может быть построено (хотя мы этого и не делаем) методами, используемыми в [3], но оно не даст полного описания группы $\operatorname{Aut}(U)$, поскольку между линками будут нетривиальные соотношения, описание которых нами на данный момент не получено.

Наконец, рассматривается случай, когда $F_{R_{1_{-}}}(V, C)=0$. Легко видеть, что в данной ситуации $(V, C)$ является правожестким объектом категории $R_{1_{-}}$, поэтому $\operatorname{Aut}(U)=\operatorname{Aut}(V, C)$.

В заключение отметим работу [11]. В этой статье авторы, если придерживаться терминологии настоящей работы, описывают класс минимальных пар $(V, C)$, не являющихся правожесткими объектами категории $R_{1}$, после чего доказывают теорему о разложении диаграммы типа "верхний домик" для рассматриваемых ими пар на элементарные линки. Отметим, что в рассуждениях относительно естественного класса пар авторы допускают неточность, утверждая, что кривая $C$ должна быть неприводима. Их доказательство теоремы о разложении на линки практически совпадает с приведенным в настоящей статье. Однако они не пишут о возможности введения композиции линков и, естественно, о соотношениях между последними.

Автор выражает благодарность ныне покойному В. А. Исковских за постановку задачи и полезные советы, а также Д. Б. Каледину и М. З. Ровинскому за прочтение работы и сделанные ими замечания.

\section{§ 2. Категории частных, жесткость, $\mathbb{Z}_{\geqslant 0}$-упорядоченные категории}

Далее везде предполагаем, что рассматриваемые категории являются малыми.

Пусть $T$ - некоторая категория, $X$ - ее подкатегория, $\Omega, \Theta$ - некоторые подмножества в $\mathrm{Ob} T$. Тогда через $T^{\vee}$ мы будем обозначать категорию, противоположную категории $T$, т. е. категорию, получаемую из $T$ обращением 
морфизмов. Очевидно, определен контравариантный функтор $\mathcal{R}_{T}: T \rightarrow T^{\vee}$, тождественный на объектах и переводящий морфизм $\varphi \in \operatorname{Mor} T$ в морфизм $\varphi^{\vee} \in \operatorname{Mor} T^{\vee}$, который имеет противоположное направление. Подмножество изоморфизмов в Mor $T$ будет обозначаться $\operatorname{Isom} T$, а изоморфизм двух объектов $V^{1}, V^{2} \in \mathrm{Ob} T-$ как $V^{1} \underset{T}{\sim} V^{2}$. Через $T(\Omega)$ обозначим полную подкатегорию в $T$, заданную условием $\mathrm{Ob} T(\Omega)=\Omega$.

Определим категории $B_{k}(T, X, \Omega), k \in \mathbb{N}$ (мы будем обозначать категории $B_{k}(T, T, \mathrm{Ob} T)$ просто $\left.B_{k}(T)\right)$, как категории, множество объектов которых $\mathrm{Ob} B_{k}(T, X, \Omega)$ состоит из диаграмм $\left(W, \varphi_{1}, \ldots, \varphi_{k}\right)$ вида

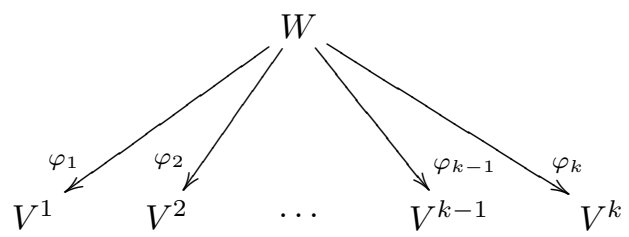

где $W \in \mathrm{Ob} X, \varphi_{i} \in \operatorname{Mor} T, V^{i} \in \Omega$ и $\left\{\varphi_{i}\right\}$ - упорядоченный набор, а множество морфизмов Mor $B_{k}(T, X, \Omega)$ состоит из морфизмов $\pi:\left(W, \varphi_{1}, \ldots, \varphi_{k}\right) \rightarrow$ $\left(W^{\prime}, \varphi_{1}^{\prime}, \ldots, \varphi_{k}^{\prime}\right)$ таких, что $\pi: W \rightarrow W^{\prime}, \pi \in \operatorname{Mor} X$ и $\varphi_{i}=\varphi_{i}^{\prime} \circ \pi$.

Композицию морфизмов мы определим естественным образом. Очевидно, что категории $B_{k}(T, X, \Omega)$ распадаются в несвязные объединения категорий $B_{k}(T, X, \Lambda)$ по всем $k$-элементным упорядоченным наборам $\Lambda$ (с возможными повторениями) в множестве $\Omega$. Отметим, что далее мы будем рассматривать только категории $B_{1}(T, X, \Omega)$ и $B_{2}(T, X, \Omega)$, являющиеся привычными категориями "стрелок" и диаграмм типа "верхний домик".

Введем функторы $\mathcal{F}_{k}: B_{k}(T, X, \Omega) \rightarrow X$, определяемые условиями

$$
\begin{gathered}
\mathcal{F}_{k}\left(W, \varphi_{1}, \ldots, \varphi_{k}\right):=W \\
\mathcal{F}_{k}\left(\pi:\left(W, \varphi_{1}, \ldots, \varphi_{k}\right) \rightarrow\left(W^{\prime}, \varphi_{1}^{\prime}, \ldots, \varphi_{k}^{\prime}\right)\right) L:=\pi: W \rightarrow W^{\prime} .
\end{gathered}
$$

Определим множества $\Theta^{\uparrow}$ и $\Theta^{\downarrow}$ условиями

$$
\Theta^{\uparrow}:=\mathcal{F}_{1}\left(\mathrm{Ob} B_{1}(T, T, \Theta)\right), \quad \Theta^{\downarrow}:=\mathcal{F}_{1}\left(\mathrm{Ob} B_{1}\left(T^{\vee}, T^{\vee}, \Theta\right)\right) .
$$

Таким образом, множество $\Theta^{\uparrow}$ состоит из всех объектов категории $T$, для которых существует морфизм в объект из множества $\Theta$, а множество $\Theta^{\downarrow}$ состоит из всех объектов категории $T$, в которые существует морфизм из объекта, принадлежащего множеству $\Theta$.

Определим категорию морфизмов $C(T, \Theta, \Omega)$ следующим образом:

$$
\begin{gathered}
\operatorname{Ob} C(T, \Theta, \Omega):=\left\{(W, \varphi) \in \operatorname{Ob} B_{1}(T, T, \Omega): W \in \Theta\right\}, \\
\operatorname{Mor} C(T, \Theta, \Omega):=\left\{\left(W, \varphi, \varphi^{\prime}\right) \in \operatorname{Ob} B_{2}(T, T, \Omega): W \in \Theta\right\},
\end{gathered}
$$

где $\left(W, \varphi, \varphi^{\prime}\right) \in \mathrm{Ob} B_{2}(T, T, \Omega)$ сопоставляется морфизм $\pi:(W, \varphi) \rightarrow\left(W, \varphi^{\prime}\right)$.

Композиция морфизмов $\left(W, \varphi, \varphi^{\prime}\right)$ и $\left(W_{1}, \varphi_{1}, \varphi_{1}^{\prime}\right)$ определена тогда и только тогда, когда $W=W_{1}$ и $\varphi^{\prime}=\varphi_{1}$, в этом случае $\left(W, \varphi, \varphi^{\prime}\right) \circ\left(W_{1}, \varphi_{1}, \varphi_{1}^{\prime}\right)=$ $\left(W, \varphi, \varphi_{1}^{\prime}\right)$. 
Категории $C(T, \mathrm{Ob} T, \mathrm{Ob} T)$ и $C(T, \Theta, \mathrm{Ob} T)$ будут обозначаться $C(T)$ и $C(T, \Theta)$ соответственно. Очевидно, что категории $C(T, \Theta, \Omega)$ распадаются в несвязные объединения категорий $C(T, W, \Omega)$ по всем $W \in \Theta$ (здесь и далее одноэлементное множество $\{W\}$ обозначается $W$ ). Заметим, что все морфизмы в категориях $C(T, \Theta, \Omega)$ являются изоморфизмами.

ОПРЕДЕЛЕНИЕ 2.1. Категория Т удовлетворяет левому правилу Оре, если для любых морфизмов $\varphi, \varphi^{\prime} \in \operatorname{Mor} T, \varphi: V \rightarrow Z, \varphi^{\prime}: V^{\prime} \rightarrow Z$, существуют морфизмы $\psi, \psi^{\prime} \in \operatorname{Mor} T$, дополняющие угол в диаграмме

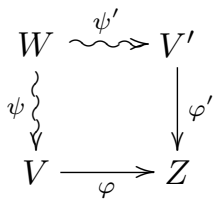

до коммутативного квадрата.

Категория $T$ удовлетворяет правому правилу Ope, если в противоположной категории $T^{\vee}$ выполнено левое правило Оре.

Здесь и далее стрелками $>$ будут обозначаться морфизмы, которые мы достраиваем.

ОПреДЕЛЕНИЕ 2.2. Пусть $T$ - некоторая категория. Введем симметричное и рефлексивное отношение $\stackrel{T}{\approx}$ следующим образом: $V \stackrel{T}{\approx} V^{\prime}$, где $V, V^{\prime} \in \mathrm{Ob} T$, если существует объект $\left(W, \varphi, \varphi^{\prime}\right)$ категории $B_{2}(T)$ такой, что $\varphi: W \rightarrow V$, $\varphi^{\prime}: W \rightarrow V^{\prime}$. Введем отношение эквивалентности $\stackrel{T}{\sim}$, являющееся продолжением отношения $\stackrel{T}{\approx}$ по транзитивности до отношения эквивалентности.

Пусть $\Omega, \Theta \subset \mathrm{Ob} T$. Через $\widetilde{\Omega}_{T}$ мы будем обозначать объединение классов эквивалентности всех объектов из $\Omega$ по модулю отношения $\stackrel{T}{\sim}$, а через $\widetilde{\Omega}_{T}^{\Theta}-$ множество $\widetilde{\Omega}_{T} \cap \Theta$.

ОПРЕДЕЛЕНИЕ 2.3. Пусть $T$ - некоторая категория, $V \in \mathrm{Ob} T$; тогда объект $V$, а также соответствующий класс эквивалентности мы будем называть правожестким (левожестким), если категория $T\left(\widetilde{V}_{T}\right)$ удовлетворяет правому правилу Оре (если категория $T\left(\widetilde{V}_{T^{\vee}}\right)$ удовлетворяет левому правилу Оре). Напомним, что $T\left(\widetilde{V}_{T}\right)$ - полная подкатегория в $T$, множеством объектов которой является класс эквивалентности объекта $V$ в категории $T$; аналогично определяется $T\left(\widetilde{V}_{T} \vee\right)$.

Подмножество правожестких объектов категории $T$ мы будем обозначать через $\mathrm{Ob}_{\text {rig }} T$.

ЗАмечАниЕ 2.4. Очевидно, если $X$ - подкатегория категории $T$, удовлетворяющая левому правилу Оре, $\Omega \subset \mathrm{Ob} T$, то во всех категориях $B_{k}(T, X, \Omega)$ выполнено левое правило Оре. Аналогичное утверждение выполнено для подкатегорий, удовлетворяющих правому правилу Оре, если вместо категорий $B_{k}(T, X, \Omega)$ рассматривать категории $\left(B_{k}\left(T^{\vee}, X^{\vee}, \Omega\right)\right)^{\vee}$.

ЗАмЕчАниЕ 2.5. 1) Поскольку $\widetilde{\Omega}_{T}=\Omega^{\uparrow \downarrow^{\uparrow \cdots}}=\Omega^{\downarrow^{\uparrow \downarrow \cdots}}=\widetilde{\Omega}_{T^{\vee}}$, отношения эквивалентности $\stackrel{T}{\sim}$ и $\stackrel{T^{\vee}}{\sim}$ совпадают, в отличие от отношений $\stackrel{T}{\approx}$ и $\underset{T^{\vee}}{\approx}$. 
2) Левожесткие объекты категории $T$ являются правожесткими объектами $T^{\vee}$, и наоборот.

3) В категории $T$ выполнено левое (правое) правило Оре тогда и только тогда, когда все ее объекты являются левожесткими (правожесткими).

УтвеРЖДЕНИЕ 2.6. Пусть категория $T$ удовлетворяет левому правилу Оре; тогда отношение $\underset{\approx}{\approx}$ является отношением эквивалентности, совпадающuм $c \stackrel{T}{\sim}$.

ДокАЗАТЕльство. Очевидно, что отношение $\stackrel{T}{\approx}$ симметрично и рефлексивно. Его транзитивность следует из достраиваемости коммутативной диаграммы

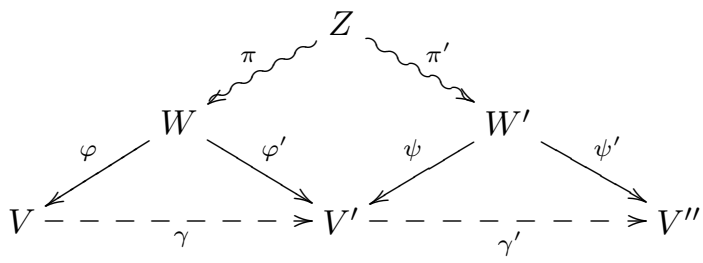

в категории $B_{2}(T)$, так как последняя удовлетворяет левому правилу Оре. Следовательно, отношение $\stackrel{T}{\approx}$ является отношением эквивалентности. Очевидно, отношение $\stackrel{T}{\approx}$ совпадает с отношением $\stackrel{T}{\sim}$.

ОПРЕДЕЛЕНИЕ 2.7. Пусть категория $T$ удовлетворяет левому правилу Оре. Введем левую категорию частных $\widetilde{T}$, задаваемую следующим набором данных:

$$
\mathrm{Ob} \widetilde{T}:=\mathrm{Ob} T, \quad \text { Mor } \widetilde{T}:=\left\{\mathrm{Ob} B_{2}(T)\right\} / \stackrel{B_{2}(T)}{\underline{ }} .
$$

Здесь морфизмами $\gamma: V \rightarrow V^{\prime}, \gamma \in \operatorname{Mor} \widetilde{T}$ (во избежании путаницы морфизмы категории $\widetilde{T}$ обозначаются пунктирными стрелками), являются классы эквивалентности объектов $\left(W, \varphi, \varphi^{\prime}\right)$ категории $B_{2}(T)$ таких, что $\varphi: W \rightarrow V$, $\varphi^{\prime}: W \rightarrow V^{\prime}$. Элементы класса $\gamma$ будут называться разрешениями морфизма $\gamma$. Композиция морфизмов $\gamma: V \rightarrow V^{\prime}$ и $\gamma^{\prime}: V^{\prime} \rightarrow V^{\prime \prime}, \gamma, \gamma^{\prime} \in \operatorname{Mor} \widetilde{T}$, в категории $\widetilde{T}$ определяется следующим условием: если $\left(W, \varphi, \varphi^{\prime}\right) \in \gamma,\left(W^{\prime}, \psi, \psi^{\prime}\right) \in \gamma^{\prime}$, то $\left(Z, \varphi \circ \pi, \varphi^{\prime} \circ \pi^{\prime}\right) \in \gamma^{\prime} \circ \gamma$, где морфизмы $\pi$ и $\pi^{\prime}$ получаются при достраивании угла, образованного морфизмами $\varphi^{\prime}$ и $\psi$ в коммутативной диаграмме $(2.4)$, до коммутативного квадрата.

Тождественные морфизмы $e_{V}: V \rightarrow V$ определяются как морфизмы в $\widetilde{T}$ такие, что $\left(V, \mathrm{id}_{V}, \mathrm{id}_{V}\right) \in e_{V}$. Очевидно, что все морфизмы в $\widetilde{T}$ обратимы: если $\left(W, \varphi, \varphi^{\prime}\right) \in \gamma$, то $\left(W, \varphi^{\prime}, \varphi\right) \in \gamma^{-1}$.

УтВЕРЖДЕНИЕ 2.8. Определение 2.7 корректно.

ДокАзАТЕльство. Для доказательства корректности определения категории $\widetilde{T}$ необходимо проверить, что класс эквивалентности, содержащий $(Z, \varphi \circ \pi$, $\left.\varphi^{\prime} \circ \pi^{\prime}\right)$ (см. диаграмму $\left.(2.4)\right)$, не зависит от выбора $\left(W, \varphi, \varphi^{\prime}\right),\left(W^{\prime}, \psi, \psi^{\prime}\right)$ в их классах эквивалентности, а также проверить ассоциативность морфизмов категории $\widetilde{T}$. Это легко сделать, используя определение 2.1.

ОПРЕДЕЛЕНИЕ 2.9. Введем функтор $\mathcal{J}_{T}: T \rightarrow \widetilde{T}$, задающий тождественное отображение на объектах и сопоставляющий морфизму $\varphi: V \rightarrow V^{\prime}, \varphi \in$ Mor $T$, морфизм $\mathcal{J}_{T}(\varphi): V \rightarrow V^{\prime}, \mathcal{J}_{T}(\varphi) \in \operatorname{Mor} \widetilde{T}$, такой, что $\left(V, \operatorname{id}_{V}, \varphi\right) \in \mathcal{J}_{T}(\varphi)$. 
ЗАмечАниЕ 2.10. 1) В случае, когда категория $T$ удовлетворяет правому правилу Оре, определена правая категория частных $\widetilde{T^{\vee}}$. Очевидно, если категория $T$ удовлетворяет обоим правилам Оре, то категории $\widetilde{T}$ и $\widetilde{T}{ }^{\vee}$ совпадают.

2) Если для любых морфизмов $\varphi, \varphi^{\prime} \in \operatorname{Mor} T$ существование $\psi \in \operatorname{Mor} T$, $\psi \circ \varphi=\psi \circ \varphi^{\prime}$, равносильно тому, что $\varphi=\varphi^{\prime}$, то функтор $\mathcal{J}_{T}$ является вложением категорий.

3) Функтор $\mathcal{J}_{T}$ является вложением.

Теорема 2.11. Пусть в категории $T$ выполнено левое правило Оре, $X$ ее полная подкатегория, удовлетворяющая следующему требованию: для любого $\varphi: V \rightarrow V^{\prime}, V^{\prime} \in \mathrm{Ob} X$, существует $\psi: V^{\prime \prime} \rightarrow V, \psi \in \operatorname{Mor} T$, такой, что $V^{\prime \prime} \in \mathrm{Ob} X$. Тогда в категории $X$ также выполнено левое правило Оре и $\widetilde{X}$ - полная подкатегория в $\widetilde{T}$, точнее, канонический функтор $\mathcal{I}_{\widetilde{X}, \widetilde{T}}: \widetilde{X} \rightarrow \widetilde{T}$ является полным и строгим. Аналогичное утверждение выполнено для категорий, удовлетворяющих правому правилу Оре, при замене указанного выше требования на противоположное.

ДокАЗАТЕЛЬСтво. Поскольку категория $X$ является полной подкатегорией в категории $T$, то $\varphi \circ \psi \in \operatorname{Mor} X$. Дальнейшее доказательство получается прямой проверкой.

ОПРЕДЕЛЕНИЕ 2.12. $\mathbb{Z}_{\geqslant 0}$-упорядоченной категорией мы будем называть категорию $T$, заданную вместе с функтором $\mathcal{P}_{T}: T \rightarrow \mathbb{Z}_{\geqslant 0}$, где $\mathbb{Z}_{\geqslant 0}-$ категория, ассоциированная с частично упорядоченным множеством $\mathbb{Z} \geqslant 0$ неотрицательных целых чисел, т. е. множество объектов категории $\mathbb{Z}_{\geqslant 0}$ совпадает с $\mathbb{Z}_{\geqslant 0}$, а множество морфизмов из $U$ в $U^{\prime}$ состоит из одного элемента, если $U \geqslant U^{\prime}$, и пусто в противном случае. Мы также будем считать, что если $\varphi: V \rightarrow V^{\prime}$, $\varphi \in \operatorname{Mor} T$, и $\mathcal{P}_{T}(V)=\mathcal{P}_{T}\left(V^{\prime}\right)$, то $\varphi \in \operatorname{Isom} T$.

ОПРЕДЕЛЕНИЕ 2.13. Пусть $T-\mathbb{Z}_{\geqslant 0}$-упорядоченная категория, $\mathcal{P}_{T}: T \rightarrow$ $\mathbb{Z}_{\geqslant 0}-$ соответствующий функтор. Тогда:

а) введем отображение $\rho_{T}:$ Mor $T \rightarrow \mathbb{Z}_{\geqslant 0}$ условием $\rho_{T}(\varphi)=\mathcal{P}_{T}(V)-\mathcal{P}_{T}\left(V^{\prime}\right)$, где $\varphi \in \operatorname{Mor} T, \varphi: V \rightarrow V^{\prime}$; заметим, что если $\varphi_{1}, \varphi_{2} \in \operatorname{Mor} T, \varphi_{1}: V^{1} \rightarrow V^{2}$, $\varphi_{2}: V^{2} \rightarrow V^{3}$, то $\rho_{T}\left(\varphi_{2} \circ \varphi_{1}\right)=\rho_{T}\left(\varphi_{2}\right)+\rho_{T}\left(\varphi_{1}\right)$

б) введем подмножества $\mathrm{Ob}^{i} T$ и $\operatorname{Mor}^{i} T$ в множествах $\mathrm{Ob} T$ и $\mathrm{Mor} T$ соответственно, где $i \in \mathbb{Z}_{\geqslant 0}$, условиями:

$$
\mathrm{Ob}^{i} T:=\left\{V \in \mathrm{Ob} T: \mathcal{P}_{T}(V)=i\right\}, \quad \operatorname{Mor}^{i} T:=\left\{\varphi \in \operatorname{Mor} T: \rho_{T}(\varphi)=i\right\} ;
$$

в) введем транзитивные отношения $\geqslant_{T}$ и $>_{T}$ на множестве $\mathrm{Ob} T$, задаваемые условиями:

$$
\begin{aligned}
& V \geqslant_{T} V^{\prime}, \quad \text { если } \exists \pi: V \rightarrow V^{\prime}, \\
& V>_{T} V^{\prime}, \quad \text { если } \exists \pi: V \rightarrow V^{\prime} \text { и } \rho_{T}(\pi)>0 ;
\end{aligned}
$$

г) введем структуры $\mathbb{Z}_{\geqslant 0}$-упорядоченных категорий на категориях $B_{k}(T$, $X, \Omega)$, где $X$ - подкатегория в $T, \Omega \subset \mathrm{Ob} T$, условиями:

$$
\begin{gathered}
\mathcal{P}_{B_{k}(T, X, \Omega)}\left(W, \varphi_{1}, \ldots, \varphi_{k}\right)=\mathcal{P}_{T}(W) \\
\mathcal{P}_{B_{k}(T, X, \Omega)}\left(\varphi:\left(W, \varphi_{1}, \ldots, \varphi_{k}\right) \rightarrow\left(W, \varphi_{1}^{\prime}, \ldots, \varphi_{k}^{\prime}\right)\right)=\mathcal{P}_{T}\left(\varphi: W \rightarrow W^{\prime}\right) .
\end{gathered}
$$


Таким образом, структура $\mathbb{Z}_{\geqslant 0}$-упорядоченных категорий на категориях $B_{k}(T, X, \Omega)$ индуцируется функторами $\mathcal{F}_{k}: B_{k}(T, X, \Omega) \rightarrow X$, определенными условиями (2.2).

ОПРЕДЕЛЕНИЕ 2.14. Пусть $T-\mathbb{Z}_{\geqslant 0}$-упорядоченная категория, $\mathcal{P}_{T}: T \rightarrow$ $\mathbb{Z}_{\geqslant 0}$. Тогда определим:

а) подмножество $\mathrm{Ob}^{M} T$ минимальных обгектов категории $T$ в множестве $\mathrm{ObT}$ условием

$$
\mathrm{Ob}^{M} T:=\left\{V \in \mathrm{Ob} T \mid \nexists V^{\prime} \in \mathrm{Ob} T, V>_{T} V^{\prime}\right\}
$$

б) множество $M_{T}(\Omega)$ минимизаций произвольного множества обгектов $\Omega \subset \mathrm{Ob} T$ категории $T$ условием

$$
M_{T}(\Omega):=\left\{\left(V^{\prime}, \pi^{\prime}\right) \mid V^{\prime} \in \mathrm{Ob}^{M} T \text { и } \exists V \in \Omega, \pi^{\prime} \in \operatorname{Mor} T, \pi^{\prime}: V \rightarrow V^{\prime}\right\} ;
$$

в) множество $N_{T, X}(V)$ минимальных разрешений обгекта $V$ в категоpuи $X$, где $X$ - подкатегория в $T$ и $V \in \mathrm{Ob} T$ (категория $B_{1}(T, X, V)$ будет называться категорией разрешений объекта $V$ в $X)$, условием

$$
N_{T, X}(V):=\mathrm{Ob}^{M} B_{1}(T, X, V)
$$

г) множество минимальных разрешений морфизма $\gamma$ в категории $T$, где категория $T$ удовлетворяет левому правилу Оре и $\gamma \in \operatorname{Mor} \widetilde{T}$, условием

$$
L_{T}(\gamma):=\gamma \cap \mathrm{Ob}^{M} B_{2}(T) .
$$

ЗАмечАниЕ 2.15 . Пусть $T-\mathbb{Z}_{\geqslant 0}$-упорядоченная категория, $\mathcal{P}_{T}: T \rightarrow \mathbb{Z}_{\geqslant 0}-$ соответствующий функтор. Тогда, поскольку любая строго убывающая последовательность объектов категории $T$ имеет минимальный элемент, множества $\mathrm{Ob}^{M} T, M_{T}(\Omega)$ при $\Omega \neq \varnothing$, а также $L_{T}(\gamma)$ для $\gamma \in \operatorname{Mor} \widetilde{T}$ в случае, если в $T$ выполнено левое правило Оре, непусты.

ТЕОРемА 2.16. $\mathbb{Z}_{\geqslant 0}$-упорядоченная категория $T$ удовлетворяет правому правилу Оре тогда и только тогда, когда для любого $V \in \mathrm{ObT}$ множество $M_{T}^{\vee}(V)$ состоит из одного класса изоморфных обгектов категории $B_{1}\left(T^{\vee}\right)$, где

$$
M_{T}^{\vee}(V):=\left\{\left(Y, \varphi^{\vee}\right) \in \mathrm{Ob} B_{1}\left(T^{\vee}\right) \mid(Y, \varphi) \in M_{T}(V), \varphi^{\vee}=\mathcal{R}_{T}(\varphi)\right\} .
$$

ДоказАтельство. Согласно замечанию 2.15 множества $M_{T}(V)$, а следовательно, и $M_{T}^{\vee}(V)$ непусты. Очевидно, что с каждым элементом вида $\left(Y, \varphi^{\vee}\right)$ множество $M_{T}^{\vee}(V)$ содержит и весь класс изоморфных ему объектов категории $B_{1}\left(T^{\vee}\right)$. Существование двух неизоморфных в $B_{1}\left(T^{\vee}\right)$ элементов $\left(Y^{1}, \varphi_{1}^{\vee}\right)$, $\left(Y^{2}, \varphi_{2}^{\vee}\right) \in M_{T}^{\vee}(V)$ противоречит выполнению правила Оре. Несложно проверить, что если для любого $V \in \mathrm{Ob} T$ множество $M_{T}^{\vee}(V)$ состоит из одного класса изоморфных объектов категории $B_{1}\left(T^{\vee}\right)$, то правое правило Оре истинно в категории $T$. Теорема доказана.

Пусть $T-\mathbb{Z}_{\geqslant 0}$-упорядоченная категория. Подмножество $\mathrm{Ob}_{\mathrm{rig}} T \cap \mathrm{Ob}^{M} T$ будем обозначать $\mathrm{Ob}_{\text {rig }}^{M} T$. Напомним, что $\mathrm{Ob}_{\text {rig }} T$ - множество правожестких объектов категории $T$.

Если $T$ удовлетворяет левому правилу Оре, то категорию $\widetilde{T}\left(\mathrm{Ob}^{M} T\right)$ будем обозначать $\widetilde{T}^{M}$. 
ЗАмечАниЕ 2.17. Пусть $T-\mathbb{Z}_{\geqslant 0}$-упорядоченная категория, $X$ - ее подкатегория такая, что структура $\mathbb{Z} \geqslant 0$-упорядоченной категории на $X$ индуцирована соответствующей структурой на $T$. Тогда очевидно, что $\mathrm{Ob}^{M} T \cap \mathrm{Ob} X \subset$ $\mathrm{Ob}^{M} X$, и если для любого $\varphi \in \operatorname{Isom} T \cap \operatorname{Mor} X$ имеем $\varphi \in \operatorname{Isom} X$, то из теоремы 2.16 следует, что $\mathrm{Ob}_{\text {rig }}^{M} T \cap \mathrm{Ob} X \subset \mathrm{Ob}_{\text {rig }}^{M} X$.

ЗАмечАниЕ 2.18. Пусть $T-\mathbb{Z}_{\geqslant 0}$-упорядоченная категория и в $T$ выполнено левое правило Оре. Тогда правожесткость минимального объекта $V$ категории $T$ означает отсутствие нетривиальных морфизмов категории частных $\widetilde{T}$ из $V$ в любой другой минимальный объект.

ОПРедЕЛЕНиЕ 2.19. Пусть $T-\mathbb{Z}_{\geqslant 0}$-упорядоченная категория, $\Theta, \Omega$ - некоторые подмножества в $\mathrm{Ob} T$ такие, что $\Theta \subset \Omega$; тогда:

1) подмножество $\Theta$ мы будем называть замкнутым в $\Omega$, если любой элемент $V$ из $\Omega$, для которого существует $W \in \mathcal{F}_{2}\left(\mathrm{Ob}^{M} B_{2}(T, T, \Theta)\right)$ такой, что $W \geqslant_{T} V$, принадлежит $\Theta$;

2) замыканием $\bar{\Theta}_{T}^{\Omega}$ подмножества $\Theta$ в $\Omega$ мы будем называть минимальное замкнутое в $\Omega$ множество, содержащее $\Theta$.

ЗАмЕчАниЕ 2.20. 1) В предположениях определения 2.19 очевидно, что $\bar{\Theta}_{T}^{\Omega} \subset \widetilde{\Theta}_{T}^{\Omega}$.

2) Отметим следующую конструкцию, объясняющую введение понятия замкнутости и очень существенную в дальнейшем. Пусть $T-\mathbb{Z}_{\geqslant 0}$-упорядоченная категория, удовлетворяющая левому правилу Ope, $\Theta \subset \Omega \subset \mathrm{Ob} T, \Theta$ замкнуто в $\Omega$ и $\gamma \in \operatorname{Mor} \widetilde{T}(\Theta)$. Предположим также, что для категории $C(T, \mathrm{Ob} T, \Omega)$ существует метод разложения морфизмов на некие "элементарные" морфизмы. Рассмотрим $\left(W, \varphi, \varphi^{\prime}\right) \in L_{T}(\gamma)$ как морфизм $\pi$ категории $C(T, \mathrm{Ob} T, \Omega)$, и пусть $\pi=\pi_{1} \circ \cdots \circ \pi_{n}$ - его разложение на элементарные. Тогда из определения $2.19,1)$ следует, что $\pi_{i} \in \operatorname{Mor} C(T, W, \Theta)$. Теперь, рассмотрев классы эквивалентности морфизмов $\pi, \pi_{1}, \ldots, \pi_{n}$ категории $B_{2}(T, T, \Omega)$, а именно $\gamma, \gamma_{1}, \ldots, \gamma_{n}$ соответственно, мы получим разложение морфизма $\gamma=\gamma_{1} \circ \cdots \circ \gamma_{n}$ такое, что $\gamma_{i} \in \operatorname{Mor} \widetilde{T}(\Theta)$.

ОПРЕДЕЛЕНИЕ 2.21. $\mathbb{Z}_{\geqslant 0}$-упорядоченную категорию $T$ мы будем называть разложимой, если любой морфизм $\varphi$ из $\operatorname{Mor}^{k} T$, где $k>0$, можно разложить в композицию $\varphi=\varphi_{1} \circ \cdots \circ \varphi_{k}$ морфизмов из $\operatorname{Mor}^{1} T$.

\section{§ 3. $\mathbb{Z}_{\geqslant 0}$-упорядоченные категории $R_{p}$-логтерминальных пар}

ОПРЕДЕЛЕНИЕ 3.1. Зададим категорию многообразий $M$ и полные подкатегории $M_{d}$ в $M$, где $d \in \mathbb{Z}, d \geqslant 0$ условиями:

$\mathrm{Ob} M:=\{$ нормальные неприводимые проективные многообразия над $\mathbb{C}$, имеющие $\mathbb{Q}$-факториальные особенности, т. е. такие, что любой дивизор Вейля является $\mathbb{Q}$-дивизором Картье\},

Mor $M:=\{$ доминантные морфизмы со связными слоями $\}$,

$\mathrm{Ob} M_{d}:=\{V \in \mathrm{Ob} M$ такие, что $\operatorname{dim} V=d\}$.

Очевидно, если $\varphi \in$ Mor $M_{d}$, то $\varphi-$ бирациональный морфизм. 
Пусть $\left(V, \pi_{1}, \ldots, \pi_{k}\right) \in \mathrm{Ob} B_{k}(M), k \in \mathbb{Z}, k \geqslant 0$. Через $Z_{j}\left(V, \pi_{1}, \ldots, \pi_{k}\right)$ обозначим группу циклов размерности $j$, лежащих в слоях морфизмов $\pi_{1}, \ldots, \pi_{k}$, где $0 \leqslant j \leqslant d, d=\operatorname{dim} V$. Поскольку $V \mathbb{Q}$-факториально, определено естественное спаривание

$$
(\cdot, \cdot): Z_{d-1}(V) \times Z_{1}\left(V, \pi_{1}, \ldots, \pi_{k}\right) \rightarrow \mathbb{Q} .
$$

Через $\equiv$ обозначим отношения численной эквивалентности на $Z_{d-1}(V)$ и $Z_{1}\left(V, \pi_{1}, \ldots, \pi_{k}\right)$.

Введем следующие понятия и обозначения:

$$
\begin{aligned}
& N_{1}\left(V, \pi_{1}, \ldots, \pi_{k}\right):=\left\{Z_{1}\left(V, \pi_{1}, \ldots, \pi_{k}\right) / \equiv\right\} \otimes \mathbb{R}, \\
& N_{d-1}(V):=\left\{Z_{d-1}(V) / \equiv\right\} \otimes \mathbb{R} .
\end{aligned}
$$

Очевидно, что спаривание (3.1) индуцирует естественное спаривание

$$
N_{1}\left(V, \pi_{1}, \ldots, \pi_{k}\right) \times N_{d-1}(V) \rightarrow \mathbb{R} .
$$

Пусть, далее, $\rho\left(V, \pi_{1}, \ldots, \pi_{k}\right):=\operatorname{dim} N_{1}\left(V, \pi_{1}, \ldots, \pi_{k}\right) ; \operatorname{NE}\left(V, \pi_{1}, \ldots, \pi_{k}\right)-$ конус в $N_{1}\left(V, \pi_{1}, \ldots, \pi_{k}\right)$, состоящий из линейных комбинаций эффективных кривых, лежащих в слоях всех морфизмов $\pi_{i}$, с коэффициентами в $\mathbb{R}_{+}$, и $\overline{\mathrm{NE}}\left(V, \pi_{1}, \ldots\right.$ $\left.\ldots, \pi_{k}\right)$ - его замыкание,

$$
\left.\overline{\mathrm{NE}}\left(V, \pi_{1}, \ldots, \pi_{k}\right)_{D \geqslant 0}:=\left\{z \in \overline{\mathrm{NE}}\left(V, \pi_{1}, \ldots, \pi_{k}\right) \mid(D, z) \geqslant 0\right)\right\},
$$

где $D \in N_{d-1}(V)$.

Подконус $Q$ в $\overline{\mathrm{NE}}\left(V, \pi_{1}, \ldots, \pi_{k}\right)$ называется экстремалъным, если существует численно эффективный класс $H \in N_{d-1}(V)$ такой, что:

$$
Q=H^{\perp} \cap \overline{\mathrm{NE}}\left(V, \pi_{1}, \ldots, \pi_{k}\right),
$$

где $H^{\perp}:=\left\{K \in N_{1}\left(V, \pi_{1}, \ldots, \pi_{k}\right) \mid(K, H)=0\right\}$.

Лучом в $\overline{\mathrm{NE}}\left(V, \pi_{1}, \ldots, \pi_{k}\right)$ назовем одномерный подконус.

$D$-экстремальным подконусом в $\overline{\mathrm{NE}}\left(V, \pi_{1}, \ldots, \pi_{k}\right)$, где $D \in N_{d-1}(V)$, называется экстремальный подконус $Q$ такой, что $(K, D)<0$ для любого $K \in Q \backslash 0$.

ОПРЕДЕлЕниЕ 3.2. Стягиванием подконуса $Q \in \overline{\mathrm{NE}}\left(V, \pi_{1}, \ldots, \pi_{k}\right)$ называется морфизм $\operatorname{cont}_{Q} \in \operatorname{Mor}, \operatorname{cont}_{Q}: V \rightarrow Z$, такой, что условие $\operatorname{cont}_{Q}(B)=\mathrm{pt}$ для кривой $B$ равносильно условию $[B] \in Q$, где $[B]-$ класс $B$ по модулю численной эквивалентности, a pt - точка. Очевидно, что морфизм $\operatorname{cont}_{Q}$ индуцирует естественно определяемый морфизм $\operatorname{cont}_{Q}:\left(V, \pi_{1}, \ldots, \pi_{k}\right) \rightarrow\left(Z, \rho_{1}, \ldots, \rho_{k}\right)$, $\operatorname{cont}_{Q} \in \operatorname{Mor} B_{k}(M)$.

Пусть $\varphi \in$ Mor $M, \varphi: V \rightarrow V^{\prime}$. Поскольку $V$ и $V^{\prime} \mathbb{Q}$-факториальны, определено отображение полного прообраза

$$
\varphi^{*}: Z_{d-1}\left(V^{\prime}\right) \otimes \mathbb{Q} \rightarrow Z_{d-1}(V) \otimes \mathbb{Q} .
$$

Если $\varphi \in \operatorname{Mor} M_{d}$, то через $\varphi_{\text {соб }}^{-1}$ и $\varphi_{*}$ обозначим стандартные отображения собственного прообраза и прямого образа:

$$
\begin{gathered}
\varphi_{\text {соб }}^{-1}: Z_{d-1}\left(V^{\prime}\right) \otimes \mathbb{Q} \rightarrow Z_{d-1}(V) \otimes \mathbb{Q}, \\
\varphi_{*}: Z_{d-1}(V) \otimes \mathbb{Q} \rightarrow Z_{d-1}\left(V^{\prime}\right) \otimes \mathbb{Q} .
\end{gathered}
$$


Пусть $V \in \mathrm{Ob} M$. Определим канонический дивизор Вейля $K_{V}$ на $V$ как продолжение канонического дивизора $K_{V \backslash \operatorname{Sing} V}$ (рассматриваемого как дивизор Вейля) с неособой части $V \backslash \operatorname{Sing} V$ многообразия $V$ на все $V$. Существование и единственность такого продолжения очевидны.

ЗАмечАниЕ 3.3. 1) Если $V \in \mathrm{Ob} M_{2}$, то определены естественные скалярные произведения $(\cdot, \cdot)$ на $Z_{1}(V) \otimes \mathbb{Q}$ и $N_{1}(V)$.

2) Если $\varphi \in \operatorname{Mor} M_{2}, \varphi: V \rightarrow V^{\prime}$, то исключительная подсхема морфизма $\varphi$, $\operatorname{Ex}(\varphi)$, является объединением конечного числа неприводимых $\varphi$-исключительных кривых и отображение $\varphi^{*}$ удовлетворяет следующему соотношению:

$$
\left(\varphi^{*} D^{\prime}, \varphi^{*} B^{\prime}\right)=\left(\varphi^{*} D^{\prime}, \varphi_{\text {соб }}^{-1}\left(B^{\prime}\right)\right)=\left(\varphi_{\text {соб }}^{-1}\left(D^{\prime}\right), \varphi^{*} B^{\prime}\right)=\left(D^{\prime}, B^{\prime}\right),
$$

где $D^{\prime}, B^{\prime} \in Z_{1}\left(V^{\prime}\right) \otimes \mathbb{Q}$. Для любой $\varphi$-исключительной кривой $E$ имеет место равенство $\left(\varphi^{*} D^{\prime}, E\right)=0$, и если $\rho(V, \varphi)=1$, то

$$
\varphi^{*} D^{\prime}=\varphi_{\text {соб }}^{-1}(D)+\left(\varphi_{\text {соб }}^{-1}(D), E\right) E .
$$

3) Если $\varphi \in \operatorname{Mor} M_{d}, \varphi: V \rightarrow V^{\prime}$, то $K_{V^{\prime}}=\varphi_{*}\left(K_{V}\right)$.

TeOpema 3.4. Пусть $V \in \mathrm{Ob} M_{2} ;$ mогда:

1) на $V$ не может существовать двух численно неэквивалентных нулю $\mathbb{Q}$-дивизоров $D_{1}$ и $D_{2}$ со свойствами $\left(D_{1}\right)^{2}>0,\left(D_{2}\right)^{2} \geqslant 0,\left(D_{1}, D_{2}\right)=0$;

2) если $\psi \in \operatorname{Mor} M_{2}, \psi: V \rightarrow V^{\prime}, u E_{i}, i \in\{1, \ldots, n\},-$ все неприводимые $\psi$-исключительные кривые, то квадратичная форма, заданная матрицей пересечения $\left(\left(E_{i}, E_{j}\right)_{i j}\right)$, отрицательно определена на подпространстве пространства $Z_{1}(V) \otimes \mathbb{Q}$, порожденном векторами $E_{i}$;

3) если $\psi$ и $E_{i}, i \in\{1, \ldots, n\}$, такие, как в $\left.n .2\right), F^{\prime}=\sum_{i=1}^{m} F_{i}^{\prime}$ - кривая на $V^{\prime}$, то квадратичная форма, заданная матрицей пересечения $\left(\left(F_{i}^{\prime}, F_{j}^{\prime}\right)_{i j}\right)$, отрицательно (неположительно) определена на подпространстве пространства $Z_{1}(V) \otimes \mathbb{Q}$, порожденном векторами $F_{i}^{\prime}$, тогда и только тогда, когда квадратичная форма, заданная матричей пересечения $\left(\left(F_{i}, F_{j}\right)_{i j}\right)$, где $F_{i}=\varphi_{\text {соб }}^{-1}\left(F_{i}^{\prime}\right)$ при $i \in\{1, \ldots, m\}, F_{i}=E_{i-m}$ при $i \in\{m+1, \ldots, m+n\}$, отрицательно (неположительно) определена на подпространстве в $Z_{1}(V) \otimes \mathbb{Q}$, порожденном векторами $F_{i}$;

4) если $V \in \mathrm{Ob} M_{2}, D_{1}, D_{2}$ - эффективные $\mathbb{Q}$-дивизоры на $V$ такие, что $\left(D_{1}\right)^{2} \geqslant 0,\left(D_{1}, D_{2}\right) \neq 0$, mо существует $a \in \mathbb{Q}$ такое, ито $\left(D_{1}+a D_{2}\right)^{2}>0$;

5) если $V \in \mathrm{Ob} M_{2}, D_{1}, D_{2}$ - эфбективные $\mathbb{Q}$-дивизоры на $V$ такие, что выполнено $\left(D_{1}\right)^{2}=\left(D_{2}\right)^{2}=\left(D_{1}, D_{2}\right)=0$, то дивизоры $D_{1}$ и $D_{2}$ численно пропорииональны.

ДоказАтельство. Пусть $\varphi: W \rightarrow V$ - разрешение особенностей поверхности $V, D_{1}^{W}=\varphi^{*}\left(D_{1}\right), D_{2}^{W}=\varphi^{*}\left(D_{2}\right)$. Тогда $\left(D_{1}^{W}\right)^{2}=\left(D_{1}\right)^{2}>0,\left(D_{2}^{W}\right)^{2}=$ $\left(D_{2}\right)^{2} \geqslant 0,\left(D_{1}^{W}, D_{2}^{W}\right)=\left(D_{1}, D_{2}\right)=0$. Индекс пересечения задает на векторном пространстве $N_{1}(W)$ квадратичную форму, которая согласно теореме Ходжа об индексе имеет тип $(1, \rho-1)$, где $\rho=\operatorname{dim} N_{1}(W)$. Если указанные в утверждении 1) дивизоры существуют, то классы $\left[D_{1}^{W}\right]$ и $\left[D_{2}^{W}\right]$ являются ненулевыми элементами в $N_{1}(W)$. Элементы $\left[D_{1}^{W}\right]$ и $\left[D_{2}^{W}\right]$ не пропорциональны, так как в противном случае найдутся отличные от нуля числа $n_{1}$ и $n_{2}$ такие, что дивизор $D^{W}=n_{1} D_{1}^{W}+n_{2} D_{2}^{W}$ численно эквивалентен нулю, но тогда $\left(D_{1}^{W}, D^{W}\right)=n_{1}\left(D_{1}^{W}\right)^{2}=0$ и $n_{1}=0$. Непропорциональность $\left[D_{1}^{W}\right]$ и $\left[D_{2}^{W}\right]$ противоречит теореме об индексе. Утверждение 1) доказано. 
Пусть $D=\sum_{i=1}^{n} a_{i} E_{i}, H^{\prime}$ - очень обильный дивизор на $V^{\prime}, H=(\psi)^{*}\left(H^{\prime}\right)$ и $a_{i}$ - произвольные рациональные числа. Очевидно, можно считать, что $H^{\prime}$ не содержит точек множества $\psi(\operatorname{Ex}(\psi))$. Предположим, что $(D)^{2} \geqslant 0$; тогда

$$
(D)^{2} \geqslant 0, \quad(H)^{2}=\left(H^{\prime}\right)^{2}>0, \quad(H, D)=0 .
$$

Получаем противоречие с утверждением 1). Утверждение 2) доказано.

Утверждение 3) очевидным образом следует из утверждения 2), поскольку для любого $D=\sum_{i=1}^{m+n} a_{i} F_{i} \in Z_{1}(V) \otimes \mathbb{Q}$ имеем

$$
D=\sum_{i=1}^{m} a_{i} \varphi^{*} F_{i}^{\prime}+\sum_{j=1}^{n} b_{j} E_{j}, \quad\left(\varphi^{*} F_{i}, E_{j}\right)=0 \quad \forall i, j .
$$

Для доказательства утверждения 4) достаточно заметить, что кривая, определяемая уравнением

$$
y=\left(D_{1}+x D_{2}\right)^{2}=\left(D_{1}\right)^{2}+2 x\left(D_{1}, D_{2}\right)+x^{2}\left(D_{2}\right)^{2},
$$

обязательно пересекается с верхней полуплоскостью.

Докажем утверждение 5). Допустим противное. Согласно утверждению 4) для произвольного эффективного дивизора $C$ такого, что $\left(C, D_{1}\right)>0$, существует $a \in \mathbb{Q}$ такое, что $\left(C+a D_{1}\right)^{2}>0$. Из утверждения 1$)$ следует, что $\left(C, D_{2}\right)=\left(C+a D_{1}, D_{2}\right) \neq 0$. Поэтому существует $b \in \mathbb{Q}$ такое, что $\left(C, D_{1}+b D_{2}\right)=0$. Тогда

$$
\left(D_{1}+b D_{2}\right)^{2}=0, \quad\left(C+a D_{1}\right)^{2}>0, \quad\left(C+a D_{1}, D_{1}+b D_{2}\right)=0 .
$$

Это противоречит утверждению 1$)$, поскольку дивизоры $C+a D_{1}$ и $D_{1}+b D_{2}$ численно неэквивалентны нулю. Теорема полностью доказана.

СлеДСтвиЕ 3.5. Пусть $\varphi \in$ Mor $M_{2}, \varphi: V \rightarrow V^{\prime}, \rho(V, \varphi)=1, E-\varphi$-исключительная кривая, $D^{\prime}$ - эбфективный $\mathbb{Q}$-дивизор на $V^{\prime}, D=\varphi_{\text {соб }}^{-1}\left(D^{\prime}\right), K, K^{\prime}-$ $\mathbb{Q}$-дивизоры на $V, V^{\prime}$ такие, что $K^{\prime}=\varphi_{*}(K),(K, E) \leqslant 0$. Тогда:

1) $(D)^{2} \leqslant\left(D^{\prime}\right)^{2}$ u $\left(D^{\prime}\right)^{2}=(D)^{2}$, если и только если $(D, E)=0$, в этом случае для любого $\mathbb{Q}$-дивизора $F$ на $V$ имеем $(F, D)=\left(\varphi_{*}(F), D^{\prime}\right)$;

2) $\left(K^{\prime}, D^{\prime}\right) \leqslant(K, D)$;

3) класс кривой $[E]$ порождает экстремалъный луч $Q$ в $\overline{\mathrm{NE}}(V)$ (действительно, $Q=L^{\perp}$, где $L=[H-(H, E) E]$, а $H$ - обильньй дивизор).

ОПРедЕЛЕНИЕ 3.6. Категорией двумернъх пар $R$ назовем категорию, объектами которой являются пары $(V, C)$ такие, что $V \in \mathrm{Ob} M_{2}, C=\sum_{i=1}^{n} C_{i}$, где $C_{i}$ - попарно различные неприводимые кривые на $V$, а морфизмами $\varphi:(V, C) \rightarrow\left(V^{\prime}, C^{\prime}\right)$ являются морфизмы $\varphi: V \rightarrow V^{\prime}, \varphi \in$ Mor $M$, такие, что $\varphi^{-1}(C)=C^{\prime}$. Мы будем также предполагать, что множество Ob $R$ coдержит ровно по одному объекту в классе изоморфных. Введем структуру $\mathbb{Z}_{\geqslant 0}$-упорядоченной категории на категории $R$ условием $\mathcal{P}_{R}(V, C):=\rho(V)$, где $\rho(V)$ - число Пикара.

ЗАмЕчАНИЕ 3.7. Мы всегда будем подразумевать, что на категориях $B_{k}(R$, $X, \Omega)$, где $X$ - подкатегория в $R, \Omega \subset \mathrm{Ob} R$, структура $\mathbb{Z} \geqslant 0$-упорядоченных категорий задана, как в определении 2.13, г). 
ОПРЕДЕЛЕНИЕ 3.8. 1) Пусть $p \in \mathbb{Q}, q \in \mathbb{Q}, 0 \leqslant p \leqslant 1,0 \leqslant q \leqslant 1$. Зададим подмножество р-морфизмов $\operatorname{Mor}_{p} R$ в $\operatorname{Mor} R$ условием

$$
\begin{aligned}
\operatorname{Mor}_{p} R:= & \left\{\varphi \in \operatorname{Mor}^{n} R, n \neq 0: \varphi=\varphi_{n} \circ \cdots \circ \varphi_{1}, \varphi_{i} \in \operatorname{Mor}^{1} R,\right. \\
& \varphi_{i}:\left(V_{i-1}, C_{i-1}\right) \rightarrow\left(V_{i}, C_{i}\right), i \in\{1, \ldots, n\}, \varphi_{i}-\text { стягивание } \\
& \left.\left(K_{V_{i-1}}+p C_{i-1}\right) \text {-экстремального луча для любого } i\right\} \cup \operatorname{Mor}^{0} R .
\end{aligned}
$$

2) Зададим левое предельное подмножество морфизмов $\operatorname{Mor}_{a_{-}} R$ при $p \rightarrow a$, где $a \in \mathbb{Q}, 0<a \leqslant 1$, и правое предельное подмножество морбизмов Mог $_{a_{+}} R$ при $p \rightarrow a$, где $a \in \mathbb{Q}, 0 \leqslant a<1$, условиями

$$
\begin{aligned}
& \operatorname{Mor}_{a_{-}} R:=\left\{\varphi \in \operatorname{Mor} R \mid \exists \varepsilon_{0}>0: \varphi \in \operatorname{Mor}_{a-\varepsilon} R, \varepsilon_{0}>\varepsilon>0, \varepsilon \in \mathbb{Q}\right\}, \\
& \operatorname{Mor}_{a_{+}} R:=\left\{\varphi \in \operatorname{Mor} R \mid \exists \varepsilon_{0}>0: \varphi \in \operatorname{Mor}_{a+\varepsilon} R, \varepsilon_{0}>\varepsilon>0, \varepsilon \in \mathbb{Q}\right\} .
\end{aligned}
$$

Далее через \# будем обозначать или пробел, или -, или + там, где подобные обозначения имеют смысл.

3) Пусть $\varphi \in \operatorname{Mor} R$. Зададим множество $J(\varphi)$ как множество значений параметра $p$, при которых $\varphi \in \operatorname{Mor}_{p} R$.

Очевидно, что определенные в 1) и 2) подмножества морфизмов являются мультипликативно замкнутыми.

ЗАмеЧАниЕ 3.9. Пусть $\varphi \in \operatorname{Mor}^{n} R$. Тогда:

$1)$ если $n=1$, то множество $J(\varphi)$, в случае его непустоты, является интервалом, содержащимся и открытым в $[0,1]_{\mathbb{Q}}$, таким, что $0 \in J(\varphi)$ или $1 \in J(\varphi)$, где $[0,1]_{\mathbb{Q}}$ - интервал из рациональных точек;

2) если $n \neq 0$, то $J(\varphi)=\bigcup_{k \in K} \bigcap_{i=1}^{n} J\left(\varphi_{i}^{k}\right)$, где объединение берется по множеству всех возможных разложений $\varphi=\varphi_{n}^{k} \circ \cdots \circ \varphi_{1}^{k}, \varphi_{i}^{k} \in \operatorname{Mor}^{1} R$, морфизма $\varphi$.

ОПРЕДЕЛЕНИЕ 3.10. 1) Зададим множество неособых nар NS условием

$$
\begin{aligned}
& \mathrm{NS}:=\{(V, C) \in \mathrm{Ob} R: V-\text { неособая поверхность, } \\
&C-\text { дивизор с простыми нормальными пересечениями }\} ;
\end{aligned}
$$

2 ) зададим подкатегорию $R_{p_{\#}}$ в $R$ условием

$$
\begin{gathered}
\text { Ob } R_{p_{\#}}:=\left\{Z \in \operatorname{Ob} R \mid \exists \varphi \in \operatorname{Mor}_{p_{\#}} R, \varphi: W \rightarrow Z, W \in \mathrm{NS}\right\}, \\
\quad \operatorname{Mor} R_{p_{\#}}:=\left\{\varphi \in \operatorname{Mor}_{p_{\#}} R, \varphi: V \rightarrow V^{\prime} \mid V, V^{\prime} \in \text { Ob } R_{p_{\#}}\right\} .
\end{gathered}
$$

Категория $R_{p}$, очевидно являющаяся разложимой, будет называться категорией р-логтерминальных пар;

3) при $(V, C) \in \mathrm{Ob} R$ определим множество $I(V, C)$ как множество значений $p$, при которых $(V, C) \in \mathrm{Ob} R_{p}$;

4) при $\varphi \in \operatorname{Mor} R$ определим множество $I(\varphi)$ как множество значений $p$, при которых $\varphi \in$ Mor $R_{p}$.

ЗАмечанИЕ 3.11. 1) Пусть $(V, C) \in \mathrm{Ob} R$; тогда $I(V, C)=\bigcup J\left(\varphi_{i}\right)$, где объединение берется по всем морфизмам $\varphi_{i}:\left(W_{i}, C^{W_{i}}\right) \rightarrow(V, C)$ таким, что $\left(W, C^{W}\right) \in \mathrm{NS} . \quad$ Пусть $\varphi \in \operatorname{Mor} R, \varphi:(V, C) \rightarrow\left(V^{\prime}, C^{\prime}\right) ;$ тогда $I(\varphi)=$ $I(V, C) \cap J(\varphi)$.

2) Из замечания 3.9 и п. 1) следует, что множества $J(\varphi), I(V, C)$ и $I\left(\varphi^{\prime}\right)$ для $\varphi \in \operatorname{Mor} R,(V, C) \in \operatorname{Ob} R$ и $\varphi^{\prime} \in$ Mor $R$ являются открытыми подмножествами B $[0,1]_{\mathbb{Q}}$. 
$3)$ Из 2) следует, что категория $R_{p}$ является подкатегорией в $R_{p_{+}}$и $R_{p_{-}}$.

4) Согласно тому, что произвольный морфизм $\varphi \in \operatorname{Mor}^{n} R, n>0$, являясь последовательностью дивизориальных стягиваний, имеет не более чем $n$ ! различных разложений на морфизмы из $\operatorname{Mor}^{1} R$, из 2) и замечания 3.9 следует, что категория $R_{p_{\#}}$ является разложимой.

Tеорема 3.12 (о конусе). 1) Пусть $\left(V, \pi_{1}, \ldots, \pi_{k}\right) \in \mathrm{Ob} B_{k}(M),(V, C) \in$ $\mathrm{Ob} R_{p}$. Тогда

$$
\overline{\mathrm{NE}}\left(V, \pi_{1}, \ldots, \pi_{k}\right)=\overline{\mathrm{NE}}\left(V, \pi_{1}, \ldots, \pi_{k}\right)_{K_{V}+p C \geqslant 0}+\sum_{i} Q_{B_{i}}
$$

где $Q_{B_{i}}-\left(K_{V}+p C\right)$-экстремальные лучи в $\overline{\mathrm{NE}}\left(V, \pi_{1}, \ldots, \pi_{k}\right)$.

2) Экстремальные лучи $Q_{B_{i}}$ дискретны в полупространстве $\overline{\mathrm{NE}}\left(V, \pi_{1}, \ldots\right.$ $\left.\ldots, \pi_{k}\right)_{K_{V}+p C<0}$, и для любого $Q_{B_{i}}$ существует неприводимая кривая $B_{i}$ такал, что $Q_{B_{i}}=\mathbb{R}_{+}\left[B_{i}\right]$, где $\left[B_{i}\right]-\kappa л а с с B_{i}$ по модулю численной эквивалентности.

ДОКАЗАТЕЛЬСТво см. в [12].

Tеорема 3.13 (о стягивании). Пусть $\left(V, \pi_{1}, \ldots, \pi_{k}\right) \in \operatorname{Ob} B_{k}(M),(V, C) \in$

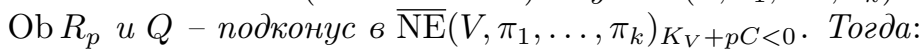

1) стягивание $\operatorname{cont}_{Q} \in \operatorname{Mor}_{M}$ подконуса $Q$ существует, если и только

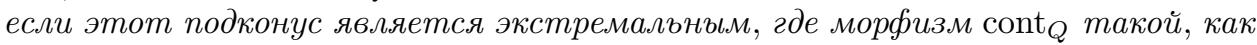
в определении 3.2 ;

2) если это стягивание дивизориально и его исключительный дивизор лежит в $C$, то морфизм $\operatorname{cont}_{Q}$ индуиирует естественно определяемыи морфизм $\operatorname{cont}_{Q} \in \operatorname{Mor} B_{k}\left(R, R_{p}\right.$, Ob $\left.R\right)$.

ДоказАтельство. Утверждение 1) доказано в [12]. Утверждение 2) легко выводится из теоремы 3.12, утверждения 1) данной теоремы и следствия $3.5,2$ ). Теорема доказана.

Tеорема 3.14. Морбизм $\varphi:(V, C) \rightarrow\left(V^{\prime}, C^{\prime}\right), \varphi \in \operatorname{Mor}^{n} R, n \neq 0$, принадлежит Mor $R_{p}$ тогда и только тогда, когда $(V, C) \in \mathrm{Ob} R_{p} u$

$$
K_{V}+p C=\varphi^{*}\left(K_{V^{\prime}}+p C^{\prime}\right)+\sum_{i=1}^{n} a_{i} E_{i}
$$

где $a_{i} \in \mathbb{Q}, a_{i}>0, u E_{i}, i \in\{1, \ldots, n\},-$ все неприводимые $\varphi$-исключительные кривые.

ДокАзАТЕльство. В одну сторону утверждение очевидно: если $\varphi \in$ Mor $R_{p}$, то $\varphi$ представляется в виде последовательности стягиваний $\left(K_{V}+p C\right)$-экстремальных лучей. Отсюда, используя очевидную индукцию, получаем доказываемое утверждение, поскольку если $\varphi:(V, C) \rightarrow\left(V^{\prime}, C^{\prime}\right)$ - стягивание $\left(K_{V}+p C\right)$-экстремального луча $Q, E$ - соответствующая ему кривая с $E^{2}<0$, то выполнено

$$
K_{V}+p C=\varphi^{*}\left(K_{V^{\prime}}+p C^{\prime}\right)+a E,
$$

где $a \in \mathbb{Q}$, так как $\left(K_{V}+p C\right)$ - $\mathbb{Q}$-дивизор Картье, и $a>0$, поскольку $\left(K_{V}+\right.$ $p C, E)=a(E)^{2}<0$. 
Докажем обратную импликацию. Согласно теореме 3.4,2) квадратичная форма, задаваемая матрицей пересечения $\left(\left(E_{i}, E_{j}\right)_{i j}\right)$, отрицательно определена. Рассмотрим соответствующую ей билинейную форму $(\cdot, \cdot)$ на векторном пространстве $V$, порожденном всеми векторами $E_{i}$. Для любого $v \in V$ имеем $(v, v)<0$. Пусть $v=\sum_{i=1}^{n} a_{i} E_{i}, a_{i} \in \mathbb{Q}, a_{i}>0$. Тогда

$$
(v, v)=\left(\sum_{i=1}^{n} a_{i} E_{i}, \sum_{i=1}^{n} a_{i} E_{i}\right)=\sum_{j=1}^{n} a_{j}\left(E_{j}, \sum_{i=1}^{n} a_{i} E_{i}\right)<0 .
$$

Следовательно, существует кривая $E_{k}$ такая, что

$$
\left(E_{k}, K_{V}+p C\right)=\left(E_{k}, \varphi^{*}\left(K_{V^{\prime}}+p C^{\prime}\right)+\sum_{i=1}^{n} a_{i} E_{i}\right)=\left(E_{k}, \sum_{i=1}^{n} a_{i} E_{i}\right)<0 .
$$

Согласно следствию $3.5,3)$ класс $\left[E_{k}\right]$ порождает экстремальный луч $Q_{k}$ в $\overline{\mathrm{NE}}(V, \varphi)$. Из теоремы 3.13 следует, что определено стягивание $\sigma_{1} \in \operatorname{Mor}^{1} R_{p}$ луча $Q_{k}, \sigma_{1}:(V, C) \rightarrow\left(V^{1}, C^{1}\right)$. Имеем

$$
\begin{aligned}
K_{V^{1}}+p C^{1} & =\sigma_{1 *}\left(K_{V}+p C\right)=\sigma_{1 *} \varphi^{*}\left(K_{V^{\prime}}+p C^{\prime}\right)+\sum_{i=1}^{n} a_{i} \sigma_{1 *} E_{i} \\
& =\left(\varphi \circ \sigma_{1}^{-1}\right)^{*}\left(K_{V^{\prime}}+p C^{\prime}\right)+\sum_{i \neq k} a_{i} E_{i}^{1},
\end{aligned}
$$

где $E_{i}^{1}=\sigma_{1 *} E_{i}$. При $n>1$ аналогично строим стягивание $\sigma_{2}:\left(V^{1}, C^{1}\right) \rightarrow$ $\left(V^{2}, C^{2}\right), \sigma_{2} \in \operatorname{Mor}^{1} R_{p}$, и т. д. В результате получаем разложение $\varphi=\sigma_{n} \circ \cdots \circ \sigma_{1}$ такое, что $\sigma_{i}:\left(V^{i-1}, C^{i-1}\right) \rightarrow\left(V^{i}, C^{i}\right),\left(V^{0}, C^{0}\right)=(V, C),\left(V^{n}, C^{n}\right)=\left(V^{\prime}, C^{\prime}\right)$, $\sigma_{i} \in \operatorname{Mor}^{1} R_{p}$. Теорема доказана.

СледСтвиЕ 3.15. Пусть $(V, C) \in \mathrm{Ob} R, \varphi:(V, C) \rightarrow\left(V^{\prime}, C^{\prime}\right), \varphi \in \operatorname{Mor}^{n} R$, $n \neq 0$. Тогда множество $I(\varphi)$ является интервалом (возможно, пустым), содержащимся и открытым в интервале $[0,1]_{\mathbb{Q}}$.

ДокАЗАТЕЛЬство. Пусть $E_{i}, i \in\{1, \ldots, n\},-$ все неприводимые $\varphi$-исключительные кривые, $p \in \mathbb{Q}$; тогда

$$
K_{V}+p C=\varphi^{*}\left(K_{V^{\prime}}+p C^{\prime}\right)+\sum_{i=1}^{n} a_{i}(p) E_{i}
$$

Очевидно, что функции $a_{i}(p)$ линейны по $p$ и множество значений $p$, где все $a_{i}(p)>0$, является пересечением конечного числа открытых полупрямых. Тогда из теоремы 3.14 следует, что множество $I(\varphi)$ имеет вид, указанный в формулировке. Следствие доказано.

ЗАмЕчАниЕ 3.16. Все построения, определения, утверждения и теоремы настоящего параграфа остаются верными при замене категории $M$ на категорию $M^{*}$, определяемую следующим образом:

$\mathrm{Ob} M^{*}:=\{$ нормальные квазипроективные многообразия над $\mathbb{C}$, имеющие $\mathbb{Q}$-факториальные особенности $\}$,

Mor $M^{*}:=\{$ проективные морфизмы со связными слоями $\}$.

В частности, можно определить категории $R_{p_{\#}^{*}}^{*}$. 


\section{§4. Свойства категорий $R_{p}$, неособые подкатегории}

ОПРЕДЕЛЕНИЕ 4.1. 1) Пусть $X$ - подкатегория категории $R$. Зададим полную подкатегорию $X^{N}$ в $X$, называемую неособой, условием

$$
\mathrm{Ob} X^{N}:=\mathrm{Ob} X \cap \mathrm{NS} .
$$

2) Поскольку категория $X^{N}$ однозначно определяется категорией $X$, объекты категории $B_{1}\left(X, X^{N},(V, C)\right)$, где $(V, C) \in \mathrm{Ob} X$, назовем разрешениями особенностей пары $(V, C)$ в категории $X$, а множества $N_{X, X^{N}}(V, C)$ - множествами минимальных разрешений особенностей пары $(V, C)$ в $X$. Последние будут обозначаться $N_{X}(V, C)$.

ЗАмечАниЕ 4.2 (1-морфизмы в категориях $R^{N}$ и $\left.R_{p}^{N}\right)$. Пусть $\varphi:(V, C) \rightarrow$ $\left(V^{\prime}, C^{\prime}\right), \varphi \in \operatorname{Mor}^{1} R^{N}$, и $\varphi$ стягивает неприводимую кривую $E$. Тогда очевидно, что $p_{a}(E)=0,(E)^{2}=-1$ и $\varphi \in \operatorname{Mor}^{1} R^{N}$, если и только если $E \subset C$ и $(E$, $C-E) \leqslant 2$. При этом $\varphi \in \operatorname{Mor} R_{p}^{N}$ тогда и только тогда, когда $(E, C-E) \leqslant 1$ при $p=1,(E, C-E) \leqslant 2$ при $p<1$.

ЗАмечАниЕ 4.3. 1) Напомним, что любой морфизм $\varphi: V \rightarrow V^{\prime}$, где $V, V^{\prime}-$ неособые поверхности, представляется в виде последовательности стягиваний $(-1)$-кривых. (Здесь и далее $(-1)$-кривой на поверхности $V$ мы будем называть кривую арифметического рода нуль, не проходящую через особенности $V$, с индексом самопересечения, равным -1.) Если $\varphi:(V, C) \rightarrow\left(V^{\prime}, C^{\prime}\right), \varphi \in \operatorname{Mor}^{1} R$, $\left(V^{\prime}, C^{\prime}\right) \in \mathrm{NS}$ и $V$ - неособая поверхность, то $\varphi \in \operatorname{Mor}^{1} R^{N}$. Отсюда следует, что категория $R^{N}$ является разложимой, и если $\varphi:(V, C) \rightarrow\left(V^{\prime}, C^{\prime}\right), \varphi \in$ Mor $R$, $\left(V^{\prime}, C^{\prime}\right) \in \mathrm{NS}$ и $V-$ неособая поверхность, то $\varphi \in \operatorname{Mor} R^{N}$.

2) Из п. 1) и замечания 4.2 следует, что $R^{N} \sim R_{p_{*}}^{N} \sim R_{1_{-}}^{N}$ при $0 \leqslant p<1$. Поэтому категории $R_{1_{-}}^{N}$ и $R_{p_{\#}}^{N}$ при $0 \leqslant p<1$, в отличие от категории $R_{1}^{N}$, являются разложимыми.

Лемма 4.4. Пусть $X-$ категория $R_{p_{\#}}$ или $R_{p_{\#}}^{N}, \varphi, \varphi^{\prime} \in \operatorname{Mor} X$, морфизм $\varphi$ является стягиванием кривой $E^{W}=\sum_{i=1}^{n} E_{i}^{W}$, а морфизм $\varphi^{\prime}-$ стягиванием кривой $\left(E^{\prime}\right)^{W}=\sum_{i=1}^{n^{\prime}}\left(E_{i}^{\prime}\right)^{W}$ (m. е. $\left.\operatorname{Ex}(\varphi)=E^{W}, \operatorname{Ex}\left(\varphi^{\prime}\right)=\left(E^{\prime}\right)^{W}\right)$. Пусть, далее, $E^{\prime}=\varphi_{*}^{\prime} E^{W}=\sum_{i=1}^{m^{\prime}} E_{i}^{\prime}, E=\varphi_{*}\left(E^{\prime}\right)^{W}=\sum_{i=1}^{m} E_{i}$ и квадратичная борма, задаваемая матрицей пересечения $\left(\left(E_{i}^{\prime}, E_{j}^{\prime}\right)_{i j}\right)$, отрицательно определена. Тогда существуют морфизмы $\psi, \psi^{\prime} \in \operatorname{Mor} X$, являющиеся стягиваниями кривых $E, E^{\prime}$ и дополняюшие угол в диаграмме

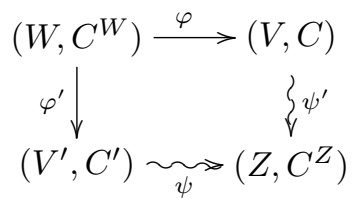

до коммутативного кодекартового квадрата.

ДокАзАтЕЛьство. Из теоремы $3.4,3)$ следует, что квадратичная форма, задаваемая матрицей пересечения $\left(\left(E_{i}, E_{j}\right)_{i j}\right)$, отрицательно определена.

Заметим, что из того, что категории $R_{p_{\#}^{N}}^{N}$ являются полными подкатегориями в $R_{p_{\#}}$, следует, что утверждение леммы достаточно доказать для следующих 
двух случаев: $X \neq R_{p_{\#}}^{N}$ и $X=R^{N}$. Заметим также, что в обоих случаях категория $X$ является разложимой, поэтому можно считать, что $\varphi, \varphi^{\prime} \in \operatorname{Mor}^{1} X$. Тогда утверждение леммы в первом случае вытекает из утверждений 1)-3) следствия 3.5, теоремы 3.13, а также того факта, что если $\operatorname{Ex}(\varphi)=\operatorname{Ex}\left(\varphi^{\prime}\right)$, то существует $\rho \in \operatorname{Isom} X$ такой, что $\varphi^{\prime}=\rho \circ \varphi$. Для доказательства леммы во втором случае достаточно воспользоваться следствием $3.5,1$ ) и замечанием 4.2, а также отметить, что при $\operatorname{Ex}(\varphi) \neq \operatorname{Ex}\left(\varphi^{\prime}\right)$ выполнено неравенство $\left(E^{\prime}, C^{\prime}-E^{\prime}\right) \leqslant\left(E^{W}, C^{W}-E^{W}\right)$. Лемма доказана.

ТеОрема 4.5. Пусть $X$ и $Y$ - или категория $R_{p_{\#}}$, или $R_{p_{\#}}^{N}$, категория $X$ является подкатегорией в $Y$ u $\Omega \subset \mathrm{Ob} Y$. Тогда категории $B_{k}(Y, X, \Omega)$ при $k>0$ удовлетворяют правому правилу Оре.

ДоказАтельСтво. Пусть $\rho, \rho^{\prime} \in \operatorname{Mor} B_{k}(Y, X, \Omega)$,

$$
\begin{aligned}
& \rho:\left(\left(Y, C^{Y}\right), \mu_{1}, \ldots, \mu_{k}\right) \rightarrow\left(\left(W, C^{W}\right), \varphi_{1}, \ldots, \varphi_{k}\right), \\
& \rho^{\prime}:\left(\left(Y, C^{Y}\right), \mu_{1}, \ldots, \mu_{k}\right) \rightarrow\left(\left(W^{\prime}, C^{W^{\prime}}\right), \varphi_{1}^{\prime}, \ldots, \varphi_{k}^{\prime}\right) .
\end{aligned}
$$

Необходимо доказать, что существуют морфизмы $\pi, \pi^{\prime} \in \operatorname{Mor} X$ и $\varepsilon_{1}, \ldots, \varepsilon_{k} \in$ Mor $Y$ такие, что диаграмма

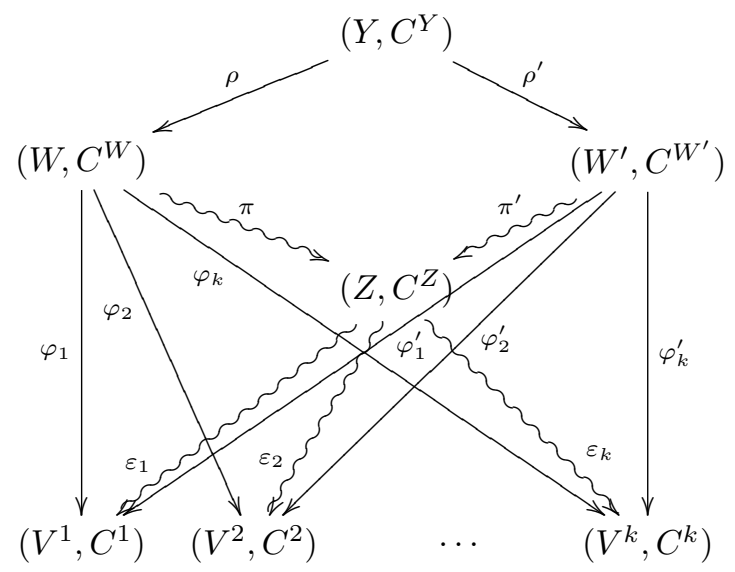

коммутативна. Очевидно, можно считать, что $\operatorname{Ex}(\rho) \neq \operatorname{Ex}\left(\rho^{\prime}\right)$ (в противном случае доказательство тривиально) и что, например, $\operatorname{Ex}(\rho) \not \subset \operatorname{Ex}\left(\rho^{\prime}\right)$. Тогда существует кривая $E^{\prime}$ на $W^{\prime}$ такая, что морфизм $\rho$ является стягиванием кривой $E^{W}$ такой, что $\rho_{*}^{\prime}\left(E^{W}\right)=E^{\prime}$. Из того, что $\rho$ стягивает кривую $E^{W}$, следует, что морфизмы $\varphi_{1}^{\prime}, \ldots, \varphi_{k}^{\prime}$ стягивают кривую $E^{\prime}$. Поэтому если $E^{\prime}=\varphi_{*}^{\prime} E^{W}=\sum_{i=1}^{m} E_{i}^{\prime}$, то согласно теореме $3.4,2)$ квадратичная форма, заданная матрицей пересечения $\left(\left(E_{i}^{\prime}, E_{j}^{\prime}\right)_{i j}\right)$, отрицательно определена и по лемме 4.4 существуют морфизмы $\pi, \pi^{\prime} \in$ Mor $X$ такие, что $\pi^{\prime}:\left(W^{\prime}, C^{W^{\prime}}, D^{W^{\prime}}\right) \rightarrow\left(Z, C^{Z}, D^{Z}\right)$ - стягивание кривой $E^{\prime}$ и $\pi \circ \rho=\pi^{\prime} \circ \rho^{\prime}$.

Положим $\varepsilon_{i}:=\varphi_{i} \circ \pi^{-1}=\varphi_{i}^{\prime} \circ\left(\pi^{\prime}\right)^{-1}$. Из леммы 4.4 следует, что $\varepsilon_{i} \in \operatorname{Mor} Y$ и диаграмма (4.2) коммутативна. Теорема доказана.

Теорема 4.6. Пусть $X$ - одна из категорий $R, R_{p_{\#}}$ при $0 \leqslant p<1, R_{1_{-}}$ или $R^{N}$. Тогда категория $X$ удовлетворяет левому правилу Оре и бунктор $\mathcal{J}_{X}: X \rightarrow \widetilde{X}$ является вложением, где $\widetilde{X}$ - категория частных категории $X$, введенная в определении 2.7. 
ДоказАтельство. Докажем, что если $\varphi:(V, C) \rightarrow\left(Z, C^{Z}\right), \varphi^{\prime}:\left(V^{\prime}, C^{\prime}\right) \rightarrow$ $\left(Z, C^{Z}\right), \varphi, \varphi^{\prime} \in \operatorname{Mor} X$, то существуют $\left(W, C^{W}\right) \in \operatorname{Ob} X$ и морфизмы $\psi, \psi^{\prime} \in$ Mor $X, \psi:\left(W, C^{W}\right) \rightarrow(V, C), \psi^{\prime}:\left(W, C^{W}\right) \rightarrow\left(V^{\prime}, C^{\prime}\right)$, такие, что $\varphi \circ \psi=\varphi^{\prime} \circ \psi^{\prime}$.

Пусть $\left(\left(Y, C^{Y}\right), \pi\right) \in N_{X}(V, C),\left(\left(Y^{\prime}, C^{Y^{\prime}}\right), \pi^{\prime}\right) \in N_{X}\left(V^{\prime}, C^{\prime}\right), \quad \varepsilon: W \rightarrow Y$, $\varepsilon^{\prime}: W \rightarrow Y^{\prime}$ - разрешение особенностей бирационального отображения $\gamma:=$ $\left(\pi^{\prime}\right)^{-1} \circ\left(\varphi^{\prime}\right)^{-1} \circ \varphi \circ \pi, \gamma: Y \rightarrow Y^{\prime}$ (здесь подразумевается разрешение особенностей по Хиронаке) и $E_{i}, i \in\{1, \ldots, n\}, E_{i}^{\prime}, i \in\{1, \ldots, m\},-$ все неприводимые $\varepsilon$ - и $\varepsilon^{\prime}$-исключительные кривые соответственно. Положим

$$
C^{W}:=\left(\varepsilon^{-1}\right)_{\text {соб }} C^{Y}+\sum_{i=1}^{n} E_{i}=\left(\varepsilon^{\prime}\right)_{\text {соб }}^{-1} C^{Y^{\prime}}+\sum_{i=1}^{m} E_{i}^{\prime} .
$$

Очевидно, что кривая $C^{W}$ корректно определена, и из замечания 4.2 и того, что $R^{N} \sim X^{N}$, следует, что $\varepsilon, \varepsilon^{\prime} \in \operatorname{Mor} X^{N} \subset$ Mor $X$ и диаграмма

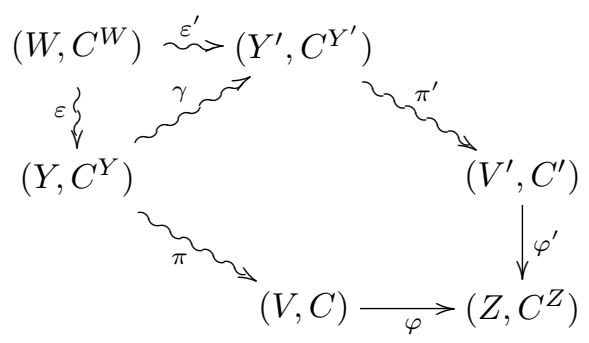

коммутативна. Осталось положить $\psi:=\pi \circ \varepsilon, \psi^{\prime}:=\pi^{\prime} \circ \varepsilon^{\prime}$. Очевидно, что $\left(W, C^{W}\right), \psi$ и $\psi^{\prime}$ удовлетворяют необходимым требованиям. Следовательно, в категории $X$ выполнено левое правило Оре. То, что функтор $\mathcal{J}_{X}$ является вложением, вытекает из замечания 2.10,3). Теорема доказана.

УтВеРЖДЕНИЕ 4.7. 1) Пусть $X$ - одна из категориц $R^{N}, R_{p_{\#}}$ при $0 \leqslant$ $p<1$ или $R_{1_{-}}$. Тогда согласно теореме 4.6 категория $X$ удовлетворяет левому правилу Оре $и$, если $\gamma \in \operatorname{Mor} \widetilde{X}$, множество $L_{X}(\gamma)$ минимальных разрешений морфизма $\gamma$ состоит из одного класса изоморбных обгектов категории $B_{2}(X)$.

2) Пусть $X$ - одна из категорий $R_{p_{\#}}$ при $0 \leqslant p<1$ или $R_{1_{-}} u(V, C) \in$ $\mathrm{Ob} X$. Тогда множество $N_{X}(V, C)$ минимальных разрешений особенностей пары $(V, C)$ состоит из одного класса изоморфных обгектов категории $B_{1}(X$, $\left.X^{N},(V, C)\right)$.

3) B предположениях n. 2) имеем $N_{X}(V, C)=N_{R}(V, C)$.

ДокАЗАтельство. Утверждение 1) непосредственно следует из того, что согласно теореме 4.5 в категории $B_{2}(X)$ выполнено правое правило Оре, и из теоремы 2.16 .

Докажем утверждение 2). Поскольку категория $X$ удовлетворяет левому правилу Оре, для любых $\left(\left(W, C^{W}\right), \varphi\right),\left(\left(W^{\prime}, C^{W^{\prime}}\right), \varphi^{\prime}\right) \in N_{X}(V, C)$ существует разрешение особенностей $\left(\left(Y, C^{Y}\right), \psi\right) \in B_{1}\left(X, X^{N},(V, C)\right)$, доминирующее разрешения $\left(\left(W, C^{W}\right), \varphi\right)$ и $\left(\left(W^{\prime}, C^{W^{\prime}}\right), \varphi^{\prime}\right)$ в $\mathbb{Z}_{\geqslant 0}$-упорядоченной категории $B_{1}\left(X, X^{N},(V, C)\right.$ ) (нужно взять разрешение морфизма $\left.\left(\varphi \circ\left(\varphi^{\prime}\right)^{-1}\right) \in \operatorname{Mor} \widetilde{X}\right)$, и $\left(\left(W, C^{W}\right), \varphi\right) \stackrel{B_{1}\left(X, X^{N},(V, C)\right)}{ }\left(\left(W^{\prime}, C^{W^{\prime}}\right), \varphi^{\prime}\right)$. Тогда из выполненности правого 
правила Оре в категории $B_{1}\left(X, X^{N},(V, C)\right)$ и теоремы 2.16 следует, что множество $N_{X}(V, C)$ состоит из одного класса изоморфных объектов категории $B_{1}\left(X, X^{N},(V, C)\right)$.

Равенство $N_{X}(V, C)=N_{R}(V, C)$ выполнено в силу того, что категория $X$ является подкатегорией в категории $R$, леммы 4.4 и $X^{N} \sim R^{N}$. Утверждение 3 ) доказано.

СлеДСтвиЕ 4.8. Пусть $(V, C) \in \mathrm{Ob} R$. Тогда согласно теореме 4.6, утверждению 4.7,2) и следствию 3.15 множество $I(V, C)$ значений $p$, где $(V, C) \in$ $\mathrm{Ob} R_{p}$, является интервалом, содержащимся и открытым в интервале $[0,1]_{\mathbb{Q}}$.

ЗАмЕчАниЕ 4.9 . Категория $R_{1}$ не удовлетворяет левому правилу Оре.

ПримеР 4.10. Пусть $V, V^{\prime}$ - неособые поверхности, $C=\sum_{i=1}^{3} C_{i}, C^{\prime}=$ $\sum_{i=1}^{3} C_{i}^{\prime}$ - кривые на $V$ и $V^{\prime}$ соответственно; $C_{i}$ и $C_{i}^{\prime}$ неособы и рациональны, $\left(C_{i}, C_{j}\right)=\left(C_{i}^{\prime}, C_{j}^{\prime}\right)=1$, если $|i-j|=1 ;\left(C_{i}, C_{j}\right)=\left(C_{i}^{\prime}, C_{j}^{\prime}\right)=0$, если $|i-j|>1$; $\left(C_{1}\right)^{2}=\left(C_{2}\right)^{2}=\left(C_{1}^{\prime}\right)^{2}=\left(C_{2}^{\prime}\right)^{2}=-1,\left(C_{3}\right)^{2}=-n,\left(C_{3}^{\prime}\right)^{2}=-(n+1), n>1$. Пусть пары $(V, C)$ и $\left(V^{\prime}, C^{\prime}\right)$ таковы, что морфизм $\varphi:(V, C) \rightarrow\left(Z, C^{Z}\right)$ является стягиванием кривых $C_{1}$ и $C_{3}$, а морфизм $\varphi^{\prime}:\left(V^{\prime}, C^{\prime}\right) \rightarrow\left(Z, C^{Z}\right)$ - последовательным стягиванием кривых $C_{3}^{\prime}$ и $C_{2}^{\prime}$. Очевидно, что $\varphi, \varphi^{\prime} \in \operatorname{Mor} R_{1}$. Легко проверить также, что не существует пары $\left(W, C^{W}\right) \in \mathrm{Ob} R_{1}$ с морфизмами $\psi:\left(W, C^{W}\right) \rightarrow(V, C), \psi^{\prime}:\left(W, C^{W}\right) \rightarrow\left(V^{\prime}, C^{\prime}\right), \psi, \psi^{\prime} \in$ Mor $R_{1}$, такими, что $\varphi \circ \psi=\varphi^{\prime} \circ \psi^{\prime}$ (для того чтобы $\varphi \circ \psi=\varphi^{\prime} \circ \psi^{\prime}$, морфизм $\psi$ должен стягивать кривую $E$ такую, что $E \subset C^{W}$ и $\left(E, C^{W}-E\right)=2$, но это невозможно в категории $\left.R_{1}\right)$.

ЗАмЕчАНИЕ 4.11. Легко привести примеры того, что множество минимальных разрешений особенностей пары $(V, C)$ в категории $R_{1}$ может содержать более одного класса изоморфных объектов категории $B_{1}\left(R_{1}, R_{1}^{N},(V, C)\right)$.

Теорема 4.12. Категория $X$, где $X-$ либо $R_{p_{\#}}, 0 \leqslant p<1$, либо $R_{1_{-}}$, является полной подкатегорией в категории $R$.

Доказательство. Пусть $(V, C),\left(V^{\prime}, C^{\prime}\right) \in \operatorname{Ob} X$ и $\varphi \in \operatorname{Mor} R$, где $\varphi$ : $(V, C) \rightarrow\left(V^{\prime}, C^{\prime}\right)$. Докажем, что $\varphi \in \operatorname{Mor} X$. Пусть

$$
\begin{gathered}
\left(\left(W, C^{W}\right), \pi\right) \in N_{R}(V, C)=N_{X}(V, C), \\
\left(\left(W^{\prime}, C^{W^{\prime}}\right), \pi^{\prime}\right) \in N_{R}\left(V^{\prime}, C^{\prime}\right)=N_{X}\left(V^{\prime}, C^{\prime}\right) .
\end{gathered}
$$

Тогда из утверждения $4.7,2)$ следует, что определен морфизм $\psi=\left(\pi^{\prime}\right)^{-1} \circ \varphi \circ \pi \in$ Mor $R^{N} \subset$ Mor $X$ и диаграмма

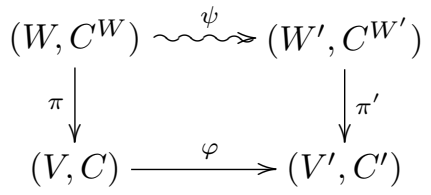

коммутативна. Из леммы 4.4 вытекает, что $\varphi \in$ Mor $X$. Теорема доказана.

ЗАмечАниЕ 4.13. Пусть категория $X$ такая, как в формулировке теоремы 4.12. Тогда: 
1) категории $B_{k}(X)$ и $C(X)$ являются полными подкатегориями в категориях $B_{k}(R)$ и $C(R)$;

2 ) из теоремы 2.11 следует, что категория $\widetilde{X}$ является полной подкатегорией в категории $\widetilde{R}$, точнее, канонический функтор $I_{\widetilde{X}}, \widetilde{R}: \widetilde{X} \rightarrow \widetilde{R}$ является строгим и полным.

\section{§ 5. 1-морфизмы в категориях $R_{p}$ и особенности объектов этих категорий}

ОПРЕДЕЛЕНИЕ 5.1. Под грабом мы будем подразумевать неориентированный граф в обычном смысле (т. е. задано множество вершин, множество ребер, и каждому ребру сопоставлены две вершины, являющиеся его концами). Будем предполагать, что некоторые вершины графа могут быть отмечены. Cтепенъю вершины мы назовем число инцидентных ей ребер.

Пусть $V \in \mathrm{Ob} M_{2}, F, G$ - связные кривые на $V, F \subset G, F=\sum_{i=1}^{n} F_{i}$, где $F_{i}$ - попарно различные неприводимые кривые на $V$, и пусть пересечения кривых $F_{i}$, как и их пересечения с $G-F$, являются нормальными.

Определим граф $\mathcal{G}(F, G)$ следующим образом: пусть $U$ - малая окрестность кривой $F$, тогда неотмеченным вершинам $\mathcal{G}(F, G)$ соответствуют кривые $F_{i}$, $i \in\{1, \ldots, n\}$, отмеченным - компоненты кривой $\left.(G-F)\right|_{U}$. Каждой точке пересечения компонент сопоставим ребро, соединяющее вершины, соответствующие пересекающимся компонентам. Отметим, что граф $\mathcal{G}(F, G)$ может иметь петли и кратные ребра. Пусть, далее, $a_{j}(F, G)$ - число неотмеченных вершин графа $\mathcal{G}(F, G)$ степени $j, k(F, G)$ - максимальное $j$ такое, что $a_{j}(F, G) \neq 0$, и $m(F, G)$ - число отмеченных вершин $\mathcal{G}(F, G)$ (очевидно, степень последних всегда равна единице).

Определим также граф $\mathcal{G}(F)$ равенством $\mathcal{G}(F)=\mathcal{G}(F, F)$.

ОПРеДЕЛЕНИЕ 5.2. Пусть $(V, C) \in \mathrm{Ob} R,\left(\left(W, C^{W}\right), \sigma\right) \in N_{R}(V, C), F \subset C-$ связная кривая на $V, F^{W}=\sum_{i=1}^{n} F_{i}^{W}=\sigma^{-1}(F)$, где $F_{i}^{W}$ - различные попарно неприводимые кривые на $W$. Тогда очевидно, что $C^{W}$ имеет простые нормальные пересечения и можно ввести следующие понятия:

1) кривая $F$ имеет mun $d_{n, m, k}$ и развернутый mun $d_{n, m, k}^{a_{0}, \ldots, a_{k}}$ в $(V, C)$, если $p_{g}\left(F_{i}^{W}\right)=0$ для любого $i, d=d\left(F^{W}\right):=p_{a}\left(F^{W}\right), a_{j}=a_{j}\left(F^{W}, C^{W}\right), m=$ $m\left(F^{W}, C^{W}\right)$ и $k=k\left(F^{W}, C^{W}\right)$;

$2)$ пара $(V, C)$ неособа в точке $x$, где $x$ - точка на $V$, если поверхность $V$ неособа в $x$ и кривая $C$ имеет простые нормальные пересечения в $x$; пара $(V, C)$ имеет особенность типа $d_{n, m, k}$ и развернутого типа $d_{n, m, k}^{a_{0}, \ldots, a_{k}}$ в точке $x$, если кривая $\sigma^{-1}(x)$ имеет тип $d_{n, m, k}$ и развернутый тип $d_{n, m, k}^{a_{0}, \ldots, a_{k}}$ в $(V, C)$.

ЗАмЕчАНИЕ 5.3. 1) В предположениях определения 5.2 очевидно, что кривая $F$ имеет тип $0_{n, m, k}$ в $(V, C)$ тогда и только тогда, когда граф $\mathcal{G}\left(F^{W}, C^{W}\right)$ является деревом.

2 ) Легко видеть, что если тип кривой отличается от $1_{2,0,2}$, то развернутый тип кривой $F$ полностью определяется ее типом, а также что $d \leqslant 1, m \leqslant 2$ и $m=0$ при $d=1$.

В случае, когда тип кривой $F$ равен $1_{2,0,2}$, имеются следующие возможности: $F$ имеет развернутый тип $1_{2,0,2}^{0,0,2}$ или $1_{2,0,2}^{0,1,1}$. 
ОПРЕДЕЛЕНИЕ 5.4. Кривую, тип которой равен $1_{n, 0, k}, k \leqslant 2$, и развернутый тип которой отличен от $1_{2,0,2}^{0,1,1}$, мы будем называть колесом.

Всюду далее мы будем предполагать, что для кривой $F$ при $k \leqslant 2$, развернутый тип которой отличен от $1_{2,0,2}^{0,1,1}$, компоненты кривой $F^{W}=\sum_{i=1}^{n} F_{i}^{W}$ занумерованы следующим образом:

$$
\left(F_{i}^{W}, F_{j}^{W}\right)=\left\{\begin{array}{c}
1, \quad \text { если }|i-j|=1(\text { или }|i-j|=n-1, \\
\left.\quad \text { когда } p_{a}\left(F_{i}^{W}\right)=1 \text { и } n \neq 1,2\right), \\
0 \quad \text { в противном случае. }
\end{array}\right.
$$

Кривая $F$ при $k \leqslant 2$ называется минималъной, если $F^{W}$ не содержит $(-1)$-кривых.

ЗАмечание 5.5. 1) С каждой кривой $F$ типа $0_{n, m, k}$ в $(V, C)$, где $k \leqslant 2$, с выбранной в определении 5.4 нумерацией на кривой $F^{W}=\sum_{i=1}^{n} F_{i}^{W}$ свяжем последовательность целых чисел $\Delta_{0}, \Delta_{1}, \ldots, \Delta_{n}$ условиями

$$
\Delta_{n}:=1 ; \quad \Delta_{i}:=\operatorname{det}\left(-\left(F_{k}^{W}, F_{j}^{W}\right)_{k j}\right) \quad \text { при } i<n, \quad \text { где } i<k, j \leqslant n .
$$

Числа $\Delta_{i}$ при $i \in\{0, \ldots, n-2\}$ удовлетворяют следующему соотношению: $\Delta_{i}=$ $-\left(F_{i}\right)^{2} \Delta_{i+1}-\Delta_{i+2}$. Отсюда легко вывести, что НОД $\left(\Delta_{i}, \Delta_{i+1}\right)=1$ при $i<n$.

$2)$ Пусть $F$ - кривая типа $d_{n, m, k}$, где $k \leqslant 2$; тогда мы будем говорить, что кривая $F$ имеет численный тuп $d_{n, m, k}\left[\left(a^{i}\right)_{i},\left(a^{j}\right)_{j}, \ldots,\left(a^{l}\right)_{l}\right]$, если $\left(F_{i}^{W}\right)^{2}=a^{i}$, $\left(F_{j}^{W}\right)^{2}=a^{j}, \ldots,\left(F_{l}^{W}\right)^{2}=a^{l}$ и $\left(F_{p}^{W}\right)^{2} \leqslant-2$ при $p \neq i, j, \ldots, l$.

Tеорема 5.6. Пусть $(V, C) \in \mathrm{Ob} R_{p}$, где $p \in \mathbb{Q}, 0 \leqslant p \leqslant 1, u B$ - приведенная кривая на $V$. Тогда существует естественно определяемый эфбективный $\mathbb{Q}$-дивизор Вейля $\operatorname{Diff}_{B}$ на $B$, называемый дифферентой, такой, что

$$
\left.\left(K_{V}+B\right)\right|_{B}=K_{B}+\operatorname{Diff}_{B} .
$$

ДоКАЗАТЕЛЬСТво следует из теоремы о логприсоединении для логтерминальных пар с $\mathbb{Q}$-факториальными особенностями (см. [13]).

ЗАмЕчАниЕ 5.7. Утверждение теоремы 5.6 ввиду своей локальности остается верным в условиях замечания 3.16 .

СлеДСтвиЕ 5.8. 1) Пусть $(V, C) \in \mathrm{Ob} R_{p}$ u $B$ - приведенная кривая на $V$ такая, что $\left(K_{V}+p C, B\right)<0, B^{2} \leqslant 0$. Тогда $p_{a}(B)=0$.

2) Любой морфизм в категории $R_{p}$ является стягиванием конечного числа деревъев, состоящих из неприводимых кривых арифметического рода нуль.

3) Пусть $(V, C) \in \mathrm{Ob} R_{p}$ и $x-$ точка на $V$. Тогда либо $x$ - неособая точка парь $(V, C)$, либо $(V, C)$ имеет особенность типа $0_{n, m, k}$ в точке $x$.

ДокАЗАТЕЛЬСтво. Утверждение 1) следует из того, что согласно теореме 5.6 имеем

$$
2 p_{a}(B)-2 \leqslant \operatorname{deg}\left(K_{B}+\operatorname{Diff}_{B}\right)=\left(K_{V}+B, B\right) \leqslant\left(K_{V}+p C, B\right)<0 .
$$

Утверждения 2) и 3) вытекают из утверждения 1), разложимости соответствующих категорий и замечания $5.3,1)$. Следствие доказано. 
Лемма 5.9. Пусть $(V, C) \in \mathrm{Ob} R, F$ - неприводимая кривая типа $0_{n, m, k}$ в $(V, C)$, где $m \leqslant 1$ u $k \leqslant 2$, содержащая не более одной особой точки пары $(V, C)$ (которая, очевидно, существует при $n>1$ и имеет тип $0_{n-1,1, k}$ ) u такая, что $(F)^{2}=-f<0$ и $F \cap(C-F) \notin \operatorname{Sing}(V)$ nри $m=1$. Пусть, далее, $\left(\left(W, C^{W}\right), \sigma\right) \in N_{R}(V, C), F^{W}=\sum_{i=1}^{n} F_{i}^{W}=\varphi^{-1}(F), F_{1}^{W}=\varphi_{\text {соб }}^{-1}(F)$, $b_{i}=-\left(F_{i}^{W}\right)^{2}$ и $\Delta_{i}$ - определители из замечания $\left.5.5,1\right)$, соответствующие кривой $F^{W}$. Тогда:

1) $f=\Delta_{0} / \Delta_{1}$

2) $\left(K_{V}+F, F\right)=\left(K_{W}+F_{1}^{W}, F_{1}^{W}\right)+\left(\Delta_{1}-1\right) / \Delta_{1}$;

3) определен морфизм $\varphi \in \operatorname{Mor} R, \varphi:(V, C) \rightarrow\left(V^{\prime}, C^{\prime}\right)$, являющийся стягиванием кривой $F$ и такой, что $J(\varphi)=(p(F), 1]_{\mathbb{Q}} \cap[0,1]_{\mathbb{Q}}$, где

$$
p(F):= \begin{cases}1-\frac{(1-m) \Delta_{1}+1}{\Delta_{0}-m \Delta_{1}} & \text { npu } \Delta_{0}-m \Delta_{1} \neq 0, p(F)<1, \\ -\infty & \text { npu } \Delta_{0}-m \Delta_{1}=0\end{cases}
$$

и если $G$ - кривая на $V$, не содержащая $F$ и такая, что $G \cap F \notin \operatorname{Sing}(V)$, $(G, F)=1 u G^{\prime}=\varphi_{*}(G)$, mo $\left(K_{V^{\prime}}+G^{\prime}, G^{\prime}\right)=\left(K_{V}+G, G\right)+\left(\Delta_{0}-1\right) / \Delta_{0}$, m. e.

$$
\operatorname{deg} \operatorname{Diff}_{G^{\prime}}=\operatorname{deg} \operatorname{Diff}_{G}+\frac{\Delta_{0}-1}{\Delta_{0}} .
$$

ДокАЗАТЕЛЬСтво. Заметим прежде всего, что $\Delta_{0}-m \Delta_{1}=0$ тогда и только тогда, когда $F-(-1)$-кривая и $m=1$. Очевидно, можно считать, что пара $(V, C)$ не имеет особенностей вне $F$. Для доказательства всех утверждений проведем индукцию по $n$.

При $n=1$ имеем $\Delta_{0}=b_{1}, \Delta_{1}=1$ и утверждения 1) и 2) очевидны:

$$
f=b_{1}=\frac{\Delta_{0}}{\Delta_{1}}, \quad\left(K_{V}+F, F\right)=-2 .
$$

Тогда $p(F)=\left(b_{1}-2\right) /\left(b_{1}-m\right)$ при $b_{1} \neq m \neq 1$ и $p(F)=-\infty$ при $b_{1}=m=1$. Из теоремы 3.13 и замечания 3.16 следует, что кривая $F$ стягиваема в категории $R$ и $J(\varphi)=(p(F), 1]_{\mathbb{Q}} \cap[0,1]_{\mathbb{Q}}$. Равенство

$$
\operatorname{deg} \operatorname{Diff}_{G^{\prime}}=\operatorname{deg} \operatorname{Diff}_{G}+\frac{b_{1}-1}{b_{1}}
$$

легко проверяется. Таким образом, в случае $n=1$ утверждения 1$)-3)$ доказаны.

Пусть при $n \leqslant k$ выполнены все утверждения леммы; докажем, что они выполнены при $n=k+1$. Из предположения индукции следует, что определено разложение морфизма $\sigma=\sigma_{2} \circ \cdots \circ \sigma_{k+1}$, где $\sigma_{i}:\left(V^{i}, C^{i}\right) \rightarrow\left(V^{i-1}, C^{i-1}\right)-$ стягивание последней $i$-й компоненты $F_{i}^{i}$ кривой $F^{i}=\left(\sigma_{i+1}\right)_{*} F^{i+1}=\sum_{j=1}^{i} F_{j}^{i}$, $\sigma_{i} \in \operatorname{Mor}^{1} R, F_{j}^{i}=\left(\sigma_{i+1}\right)_{*} F_{j}^{i+1}$ при $j \leqslant i,\left(W, C^{W}\right)=\left(V^{k+1}, C^{k+1}\right)$. Из утверждения 1) и предположения индукции получаем $\left(F_{2}^{2}\right)^{2}=-\Delta_{1} / \Delta_{2}$. Используя предположение индукции в утверждении 3$)$, имеем

$$
\left(K_{V}+F, F\right)=\left(K_{W}+F_{1}^{W}, F_{1}^{W}\right)+\frac{\Delta_{1}-1}{\Delta_{1}} .
$$


Таким образом, доказано утверждение 2) при $n=k+1$, а также то, что

$$
f=b_{1}-\frac{1}{\left(F_{2}^{2}\right)^{2}}=b_{1}-\frac{\Delta_{2}}{\Delta_{1}}=\frac{b_{1} \Delta_{1}-\Delta_{2}}{\Delta_{1}}=\frac{\Delta_{0}}{\Delta_{1}} .
$$

Таким образом, утверждение 1$)$ для $n=k+1$ доказано.

Докажем утверждение 3 ) для $n=k+1$. Из утверждений 1) и 2), замечания 3.16 и теоремы 3.13 следует, что $\varphi \in \operatorname{Mor} R$, и для интервала $J(\varphi)$ получаются границы, указанные в формулировке леммы. Мы также имеем

$$
\begin{aligned}
\left(K_{V^{\prime}}+G^{\prime}, G^{\prime}\right) & =\left(\varphi^{*}\left(K_{V^{\prime}}+G^{\prime}\right), G\right)=\left(K_{V}+\frac{\left(K_{V}, F\right)}{f} F+G+\frac{1}{f} F, G\right) \\
& =\left(K_{V}+G, G\right)+\frac{1}{f}+1+\frac{1}{f}\left(K_{V}+F, F\right) \\
& =\left(K_{V}+G, G\right)+\frac{\Delta_{1}}{\Delta_{0}}+1-\frac{\Delta_{1}}{\Delta_{0}}\left(\frac{\Delta_{1}+1}{\Delta_{1}}\right) \\
& =\left(K_{V}+G, G\right)+\frac{\Delta_{0}-1}{\Delta_{0}} .
\end{aligned}
$$

Таким образом, утверждение 3) доказано. Лемма полностью доказана.

ЗАмечАнИЕ 5.10. 1) Пусть $(V, C) \in \mathrm{Ob} R, F=\sum_{i=1}^{n} F_{i}-$ кривая типа $0_{n, m, k}$ в $(V, C)$, где $m \leqslant 1, k \leqslant 2$, такая, что форма пересечения $\left(\left(F_{i}, F_{j}\right)_{i j}\right)$ отрицательно определена, $F_{i} \cap\left(C-F_{i}\right) \cap \operatorname{Sing}(V)=\varnothing$, и если $m=1$, то $\left(F_{1}, C-F_{1}\right)=1$. Тогда, пользуясь леммой 5.9 , теоремой $3.4,3)$ и леммой 4.4 , несложно доказать, что определено стягивание $\varphi \in$ Mor $R$ кривой $F$ и $1 \in J(\varphi)$.

2) Пусть $(V, C) \in \mathrm{Ob} R, F^{1}, \ldots, F^{s}$ - минимальные кривые типов $0_{n^{j}, m^{j}, k^{j}}$ в $(V, C), j \in\{1, \ldots, s\}$, удовлетворяющих требованиям п. 1) и такие, что при $j \neq l$ имеем $\left(F_{r}^{j}, F_{t}^{l}\right)=0$ при любых $r$ и $t$. Пусть $\Delta_{i}^{j}, j \in\{1, \ldots, s\},-$ определители из замечания $5.5,1)$, соответствующие этим кривым, $G$ - кривая на $V$, не содержащая компонент кривых $F^{j}$ и такая, что $\left(G, F^{j}\right)=\left(G, F_{1}^{j}\right)=1$ и $G \cap F \notin \operatorname{Sing}(V)$. Тогда определено стягивание $\varphi:(V, C) \rightarrow\left(V^{\prime}, C^{\prime}\right)$ всех кривых $F^{j}, \varphi \in \operatorname{Mor} R$ и $1 \in J(\varphi)$. Пусть $G^{\prime}=\varphi_{*} G$. Тогда в силу того, что $\Delta_{0}^{j} \geqslant 2$, имеем

$$
\operatorname{deg} \operatorname{Diff}_{G}+s>\operatorname{deg} \operatorname{Diff}_{G^{\prime}}=\operatorname{deg} \operatorname{Diff}_{G}+\sum_{j=1}^{s} \frac{\Delta_{0}^{j}-1}{\Delta_{0}^{j}} \geqslant \operatorname{deg} \operatorname{Diff}_{G}+\frac{s}{2} .
$$

3) В условиях п. 1) пусть $F$ - кривая численного типа $0_{n, m, k}\left[(2)_{1},(2)_{2}, \ldots\right.$ $\left.\ldots,(2)_{n}\right]$ в $(V, C)$ и $\varphi$ - ее стягивание; тогда несложно проверить, что $\Delta_{i}(F)=$ $n+1-i$ и $J(\varphi)=(0,1]_{\mathbb{Q}}$.

4) В условиях п. 1) пусть $F$ - минимальная кривая численного типа $0_{n, m, k}\left[\left(a^{1}\right)_{1},\left(a^{2}\right)_{2}, \ldots,\left(a^{n}\right)_{n}\right]$ в $(V, C)$ такая, что существует $a^{i} \neq 2$, а $\varphi-$ eе стягивание; тогда легко убедиться в том, что $J(\varphi)=\left(p_{0}, 1\right]_{\mathbb{Q}}$, где $p_{0}>1 / 3$.

Tеорема 5.11. Пусть $(V, C) \in \mathrm{Ob} R_{p}$, где $p>3 / 4, x-$ mочка на $V$ такая, что через $x$ проходит больше одной ветви кривой $C$. Тогда $x$ - неособая точка nары $(V, C)$.

ДокАЗАтельство. Допустим, что $x$ - особая точка $(V, C)$. Очевидно, можно считать, что $x$ - единственная особая точка этой пары. Пусть $\left(\left(W, C^{W}\right), \sigma\right) \in$ 
$N_{R}(V, C), \sigma=\sigma_{1} \circ \cdots \circ \sigma_{n}$ - разложение морфизма $\sigma$ на морфизмы $\sigma_{i} \in \operatorname{Mor}^{1} R_{p}$, $\sigma_{i}:\left(V^{i}, C^{i}\right) \rightarrow\left(V^{i-1}, C^{i-1}\right),\left(V^{0}, C^{0}\right)=(V, C),\left(V^{n}, C^{n}\right)=\left(W, C^{W}\right), \sigma_{i}-$ стягивание неприводимой кривой $E^{i} \subset C^{i}$. Пусть $\sigma_{j}$ - первое стягивание кривой $E^{j}$, пересекающей $r>1$ ветвей кривой $C^{j}$ (очевидно, что такое стягивание всегда существует и $E^{j}$ пересекает $C^{j}-E^{j}$ в $r$ попарно различных неособых точках поверхности $V^{j}$ ). Тогда если $E^{j}$ не содержит особых точек поверхности $V^{j}$, то при $p>3 / 4$ имеем $r=\left(E^{j}, C^{j}-E^{j}\right)=2$, и из минимальности разрешения $\left(\left(W, C^{W}\right), \sigma\right)$ вытекает, что $\left(E^{j}\right)^{2} \leqslant-2$. Легко проверяется, что в этом случае $\sigma_{j} \notin \operatorname{Mor}^{1} R_{p}$. Следовательно, кривая $E^{j}$ должна проходить через особые точки $x_{1}, \ldots, x_{s}, s \geqslant 1$, поверхности $V^{j}$.

Заметим, что из условия, налагаемого на морфизм $\sigma^{j}$, вытекает, что пара $\left(V^{j}, C^{j}\right)$ имеет особенности типов $0_{n^{1}, 1, k^{1}}, \ldots, 0_{n^{s}, 1, k^{s}}$ в точках $x_{1}, \ldots, x_{s}$, где $k^{f} \leqslant 2$ при $1 \leqslant f \leqslant s$, и что $\left(E^{j}, C^{j}-E^{j}\right) \in \mathbb{Z},\left(E^{j}, C^{j}-E^{j}\right) \geqslant 2$. Тогда из замечания $5.10,2)$ получаем

$$
\begin{aligned}
\left(K_{V^{j}}+p C^{j}, E^{j}\right) & \geqslant 2 p+\left(K_{V^{j}}+p E^{j}, E^{j}\right) \\
& \geqslant 2 p+\left(K_{W}+E^{W}, E^{W}\right)+\frac{s}{2}-(1-p)\left(E^{j}\right)^{2} \\
& =2 p-2+\frac{s}{2}-(1-p)\left(E^{j}\right)^{2}>2 p-2+\frac{s}{2}>\frac{s}{2}-\frac{1}{2} \geqslant 0 .
\end{aligned}
$$

(Здесь $E^{W}$ - собственный прообраз $E^{j}$ на $W$.) Поэтому $\sigma_{j} \notin$ Mor $R_{p}$. Противоречие. Следовательно, $x$ - неособая точка пары $(V, C)$. Теорема доказана.

Tеорема 5.12 (элементарные морфизмы в категории $R_{p}$ при $p>4 / 5$ ). Пусть $(V, C) \in \mathrm{Ob} R_{p}$, где $4 / 5<p<1, E$ - неприводимая кривая на $V$, стягиваемая в категории $R_{p}, \varphi:(V, C) \rightarrow\left(V^{\prime}, C^{\prime}\right)$ - ее стягивание, $x_{1}, \ldots, x_{s}-$ все особые точки пары $(V, C)$, лежащие на кривой $E, u\left(\left(W, C^{W}\right), \sigma\right) \in N_{R}(V, C)$. Тогда $p_{a}(E)=0$. Пусть $m:=(E, C-E)$. Тогда $m=0,1,2$. Точки пересечения $E$ с $C-E$ являются неособъми точками пары $(V, C)$, все $x_{1}, \ldots, x_{s}$ имеют особенности типов $0_{n^{1}, 1, k^{1}}, \ldots, 0_{n^{s}, 1, k^{s}}$ в $(V, C)$, где $k^{l} \leqslant 2$ при $1 \leqslant l \leqslant s$. Тогда для кривой $Е$ и морфизма $\varphi$ имеются следующие возможности:

1) при $m=2: s=0,(E)^{2}=-1$, точки пересечения $E$ с $C-E$ попарно различны и $J(\varphi)=[0,1)_{\mathbb{Q}}$;

2) при $m=1: s \leqslant 1 u 1 \in J(\varphi)$;

3) при $m=0: s \leqslant 3 u 1 \in J(\varphi)$.

ДоКАЗАТЕЛЬСтво. Согласно следствию 5.8 имеем $p_{a}(E)=0$. Неравенство $m \leqslant 2$ следует из теоремы 5.11. Очевидно, можно предполагать, что пара $(V, C)$ не имеет особенностей вне $E$.

Пусть $\sigma=\sigma_{1} \circ \cdots \circ \sigma_{n}$ - разложение морфизма $\sigma$ на морфизмы $\sigma_{i} \in \operatorname{Mor}^{1} R_{p}$, $\sigma_{i}:\left(V^{i}, C^{i}\right) \rightarrow\left(V^{i-1}, C^{i-1}\right),\left(V^{0}, C^{0}\right)=(V, C),\left(V^{n}, C^{n}\right)=\left(W, C^{W}\right), \sigma_{i}-$ стягивание кривой $E^{i}$. Из теоремы 5.11 и минимальности разрешения $\left(\left(W, C^{W}\right), \sigma\right)$ вытекает, что $\left(E^{i}, C^{i}-E^{i}\right)=1$ и точка пересечения $E^{i}$ с $C^{i}-E^{i}$ является неособой точкой пары $(V, C)$. Докажем следующее вспомогательное утверждение. Предположим, что на кривой $E^{i}$ лежит не более одной особой точки $x^{i}$ пары $\left(V^{i}, C^{i}\right)$ и точка $x^{i}$ имеет тип $0_{n^{i}, 1, k^{i}}$, где $k^{i} \leqslant 2$. Тогда все особые точки $x_{1}, \ldots, x_{s}$ пары $(V, C)$, лежащие на $E$, имеют особенности типов $0_{n^{1}, 1, k^{1}}, \ldots, 0_{n^{s}, 1, k^{s}}$, где $k^{l} \leqslant 2$ при $1 \leqslant l \leqslant s$. Проведем индукцию по $n-i$.

При $i=n$ утверждение очевидно. 
Пусть при $i>j$ на кривой $E^{i}$ лежит не более одной особой точки пары $\left(V^{i}, C^{i}\right)$ типа $0_{n^{i}, 1, k^{i}}$, где $k^{i} \leqslant 2$. Заметим, что тогда все особые точки $x^{j, 1}, \ldots, x^{j, r}$ пары $\left(V^{j}, C^{j}\right)$, лежащие на кривой $E^{j}$, имеют особенности типов $0_{n^{j, 1}, 1, k^{j, 1}}, \ldots, 0_{n^{j, r}, 1, k^{j, r}}$, где $k^{j, f} \leqslant 2$ при $1 \leqslant f \leqslant r$. Из стягиваемости кривой $E^{j}$ в категории $R_{p}$ получаем

$$
\begin{aligned}
0 & >\left(K_{V^{j}}+p C^{j}, E^{j}\right)=p+\left(K_{V^{j}}+E^{j}, E^{j}\right)-(1-p)\left(E^{j}\right)^{2} \\
& =p+\left(K_{W}+E_{j}^{W}, E_{j}^{W}\right)+\sum_{f=1}^{r} \frac{\Delta_{0}^{j, f}-1}{\Delta_{0}^{j, f}}-(1-p)\left(E^{j}\right)^{2} \\
& =p-2-(1-p)\left(E^{j}\right)^{2}+\sum_{f=1}^{r} \frac{\Delta_{0}^{j, f}-1}{\Delta_{0}^{j, f}}
\end{aligned}
$$

$\left(E_{j}^{W}\right.$ - собственный прообраз $E^{j}$ на $W, \Delta_{0}^{j, f}-$ определители, соответствующие точкам $\left.x^{j, f}\right)$.

Заметим, что при $p>4 / 5,\left(E^{j}\right)^{2}<0$ и $\left(\Delta_{0}^{j, f}-1\right) / \Delta_{0}^{j, f} \geqslant 1 / 2$ имеем $r \leqslant 2$. Докажем, что $r \leqslant 1$. Допустим противное. Очевидно, что минимум выражения $\sum_{f=1}^{2}\left(\Delta_{0}^{j, f}-1\right) / \Delta_{0}^{j, f}$ достигается при $\Delta_{0}^{j, 1}=\Delta_{0}^{j, 2}=2\left(\right.$ если $\Delta_{0}^{j, f}=1$, то $x^{j, f}$ - неособая точка пары $(V, C)$ и разрешение $\left(\left(W, C^{W}\right), \sigma\right)$ не минимально). Легко видеть, что в этом случае $\left(E^{j}\right)^{2}=\left(E_{j}^{W}\right)^{2}+1$. Поэтому $\left(E^{j}\right)^{2} \in \mathbb{Z}$ и из стягиваемости $E^{j}$ следует, что $\left(E^{j}\right)^{2} \leqslant-1$. Получаем

$$
0>p-2-(1-p)\left(E^{j}\right)^{2}+\sum_{f=1}^{2} \frac{\Delta_{0}^{j, f}-1}{\Delta_{0}^{j, f}} \geqslant p-2+1-p+1=0 .
$$

Следовательно, случай $\Delta_{0}^{j, 1}=\Delta_{0}^{j, 2}=2$ невозможен. Поэтому

$$
\sum_{f=1}^{2} \frac{\Delta_{0}^{j, f}-1}{\Delta_{0}^{j, f}} \geqslant \frac{3-1}{3}+\frac{2-1}{2}=\frac{7}{6} .
$$

В этом случае $\left(E_{j}\right)^{2}=-1 / 6$. Тогда из неравенства (5.1) получаем

$$
0>p-2-(1-p)\left(E^{j}\right)^{2}+\sum_{f=1}^{2} \frac{\Delta_{0}^{j, f}-1}{\Delta_{0}^{j, f}}>\frac{5}{6} p-\frac{4}{6}>0 .
$$

Противоречие. Следовательно, этот случай невозможен. Легко проверить, что другие варианты также невозможны. Поэтому на кривой $E^{j}$ лежит не более одной особой точки $x^{j}$ пары $(V, C)$, имеющей тип $0_{n^{j}, 1, k^{j}}$, где $k^{j} \leqslant 2$, и наше вспомогательное утверждение доказано.

Пусть $E^{W}=\sigma_{\text {соб }}^{-1} E, \Delta_{0}^{l}-$ определитель, соответствующий $x_{l}, l \in\{1, \ldots, s\}$. Имеем

$$
0>\left(K_{V}+p C, E\right)=m p+\left(K_{W}+E^{W}, E^{W}\right)+\sum_{l=1}^{s} \frac{\Delta_{0}^{l}-1}{\Delta_{0}^{l}}-(1-p)(E)^{2} .
$$

По теореме 5.11 на кривой $E$ может лежать только одна особая точка численного типа $0_{n, m, k}\left[(2)_{1},(2)_{2}, \ldots,(2)_{n}\right],\left(E^{W}\right)^{2} \equiv-1$. Тогда согласно замечанию $5.10,3)$ имеем $\Delta_{0}=n+1, \Delta_{1}=n$ и по лемме 5.9 получаем, что 
$(E)^{2}=-1 /(n+1)$. Подставляя эти формулы в $(5.2)$, мы получаем противоречие с имеющимся в (5.2) неравенством. Следовательно, этот случай невозможен. Тогда из теоремы 5.11 следует, что $(E)^{2}=-1$ и на $E$ не лежат особые точки пары $(V, C)$. Таким образом, для случая $n=2$ теорема доказана.

Доказательство того, что $s \leqslant 1$ при $m=1$, аналогично приведенному выше. Из леммы $5.9,3)$ вытекает, что интервал $J(\varphi)$ имеет вид, указанный в формулировке теоремы.

Если $m=0$, то из формулы (5.2) и замечания 5.10,2), следует, что $s \leqslant 3$ и интервал $J(\varphi)$ имеет вид, указанный в формулировке. Теорема доказана.

СледСТВИЕ 5.13. 1) Согласно тому, что категория $R_{p_{\#}}\left(\widetilde{R}_{p_{\#}}\right)$ является полной подкатегорией в $R(\widetilde{R})$, из замечаний $5.10,3), 4), 3.9,2)$ и теоремы 5.12 следует, что:

а) категория $R_{p_{1 \#}}$ является полной подкатегорией в категории $R_{p_{2 \#}}$ при $4 / 5<p_{1}<p_{2}<1$, и обе они являются полными подкатегориями в категории $R_{1_{-}}$, категория $R_{p_{-}} \sim R_{p}$ является полной подкатегорией в категории $R_{p_{+}}$при $4 / 5<p<1$; аналогичные утверждения выполнены для категорий частных;

б) категория $R_{p_{1 \#}}$ является полной подкатегорией в категории $R_{p_{2 \#}}$ при $0<p_{2}<p_{1}<1 / 3$, и обе они являются полными подкатегориями в категории $R_{0_{+}}$, категория $R_{p_{+}} \sim R_{p}$ является полной подкатегорией в категории $R_{p_{-}}$при $0<p<1 / 3 ;$ аналогичные утверждения выполнены для категорий частных.

2) Из n. 1) имеем $\mathrm{Ob} R_{1_{-}}=\mathrm{Ob} R_{1}$.

3) Пусть $(V, C) \in \mathrm{Ob} R_{p_{\#}}$ при $p>4 / 5, x$ - точка на $V$. Тогда либо $x$ - неособая точка пары $(V, C)$, либо $(V, C)$ имеет особенность типов $0_{n, 0, k}, 0_{n, 1, k}$, где $k \leqslant 2$, или расширенного типа $0_{n, 0,3}^{0,3, n-4,1}$ в точке $x$. Графы, соответствующие данным типам особых точек, имеют вид
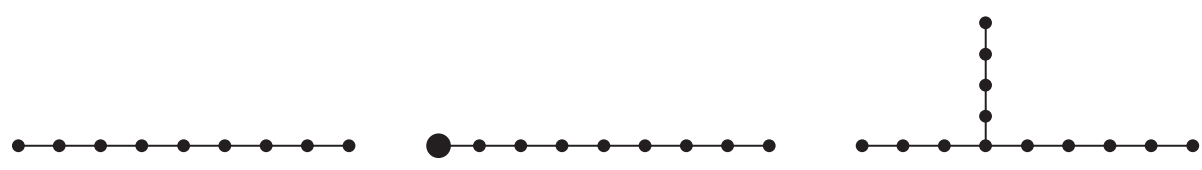

(здесь отмеченным вериинам соответствуют жирные точки).

Из замечания $5.10,1)$ следует, что на объектах категорий $R_{1_{-}}$и $R_{1}$ реализуются все возможные в категории $R$ особенности типов $0_{n, 0, k}$ и $0_{n, 1, k}$ при $k \leqslant 2$.

\section{§ 6. Функторы разрешения особенностей и минимизации. Теорема о замкнутости}

Лемма 6.1. Пусть $X$ - одна из категорий $R_{p_{\#}}$ при $4 / 5<p<1$ или $R_{1_{-}}$, $(V, C) \in \operatorname{Ob} X,\left(\left(W, C^{W}\right), \varphi\right) \in N_{X}(V, C), B \subset C$ - неприводимая компонента кривой $C$ и $\left(B^{W}, C^{W}-B^{W}\right) \leqslant 2$, где $B^{W}=\varphi_{\text {соб }}^{-1}$. . Тогда если $B^{2} \geqslant 0 u$ $\left(B^{W}\right)^{2}<0, m o$

$$
\left(B^{W}\right)^{2}=-1, \quad\left(B^{W}, C^{W}-B^{W}\right)=2, \quad(B, C-B)=0,
$$


на кривой $B$ лежат две особые точки пары $(V, C)$ типов $0_{n_{1}, 1, k_{1}} \quad$ u $0_{n_{2}, 1, k_{2}}$, $k_{1}, k_{2} \leqslant 2, u\left(W, C^{W}\right) \notin \mathrm{Ob}^{M} R^{N}$.

ДоказАтельство. Согласно теореме 5.12 пара $(V, C)$ не может иметь на кривой $C$ никаких особенностей, кроме особых точек типа $0_{n_{j}, 1, k_{j}}$, где $k_{j} \leqslant 2$. Пусть $\left(B^{W}, C^{W}-B^{W}\right)=m,(B, C-B)=n$, на кривой $B$ лежат $k$ особых точек пары $(V, C)$ типа $0_{n_{j}, 1, k_{j}}$ и $\Delta_{i}^{j}, j \in\{1, \ldots, k\},-$ определители (см. замечание $5.5,1)$, соответствующие кривым, стягиваемым в эти точки. Из того, что $\left(\left(W, C^{W}\right), \varphi\right) \in N_{X}(V, C)$ и из замечания $\left.5.10,1\right)$ следует, что $m=k+n$ и $\Delta_{i}^{j} / \Delta_{i+1}^{j}<1$. Тогда из следствия $\left.3.5,1\right)$ и леммы $\left.5.9,1\right)$ вытекает, что

$$
0 \leqslant B^{2}=\left(B^{W}\right)^{2}+\sum_{j=1}^{k} \frac{\Delta_{1}^{j}}{\Delta_{0}^{j}}<\left(B^{W}\right)^{2}+k .
$$

Отсюда и из того, что $\left(B^{W}\right)^{2}<0, k \leqslant m \leqslant 2$, получаем $\left(B^{W}\right)^{2}=-1, k=m=2$, $n=m-k=0$. Лемма доказана.

ТеОрема 6.2. Пусть $X$ - одна из категорий $R_{p_{\#}}$ при $4 / 5<p<1$ или $R_{1_{-}}$, $(V, C) \in \mathrm{Ob}^{M} R^{N} u\left(\left(Y, C^{Y}\right), \sigma\right),\left(\left(Y^{\prime}, C^{Y^{\prime}}\right), \psi\right) \in M_{X}(V, C)$, где $M_{X}(V, C)$ множество минимизаций пары $(V, C)$ из определения 2.14,б). Тогда $\operatorname{Ex}(\sigma)=$ $\operatorname{Ex}(\psi)$, множество $M_{X}^{\vee}(V, C)$ (cм. теорему 2.16) состоит из одного класса изоморфных обгектов категории $B_{1}\left(X^{\vee}\right)$ u $\left(\left(Y, C^{Y}\right), \sigma^{\vee}\right) \in M_{X}^{\vee}(V, C)$, если и только если $((V, C), \sigma) \in N_{X}\left(Y, C^{Y}\right)$.

ДокАЗАТЕЛЬСтво. Поскольку все рассуждения локальны, можно считать, что $C$ - связная кривая. Пусть $\sigma=\sigma_{1} \circ \cdots \circ \sigma_{n}$, где $\sigma_{i} \in \operatorname{Mor}^{1} X, \sigma_{i}:\left(V^{i}, C^{i}\right) \rightarrow$ $\left(V^{i-1}, C^{i-1}\right),\left(V^{n}, C^{n}\right)=(V, C),\left(V^{0}, C^{0}\right)=\left(Y, C^{Y}\right), \sigma_{i}$ - стягивание кривой $E^{i}$, а $n_{i}$ - число неприводимых компонент кривой $C^{i}$. Компоненты кривой $C^{i}$ будут обозначаться далее через $B^{i}, E^{i}, \ldots$, a их собственные прообразы на $V$ через $B_{i}^{V}, E_{i}^{V}, \ldots$.

Из связности $C$ следует, что если $n_{i}>1$, то для любой неприводимой компоненты $B^{i}$ кривой $C^{i}$ выполнено $\left(B^{i}, C^{i}-B^{i}\right) \geqslant 1$, а из теоремы 5.12 и того, что $(V, C) \in \mathrm{Ob}^{M} R^{N}$, вытекает, что если $n_{i}>1$, то $\left(E^{i}, C^{i}-E^{i}\right)=1,\left(E_{i}^{V}\right)^{2}<-1$ и на $E^{i}$ лежит не более одной особой точки пары $\left(V^{i}, C^{i}\right)$ типа $0_{n_{i}, 1, k_{i}}$, где $k_{i} \leqslant 2$.

Очевидно, что для доказательства того, что $\operatorname{Ex}(\sigma)=\operatorname{Ex}(\psi)$, достаточно доказать следующее утверждение: пусть $B^{i} \neq E^{i}$ - неприводимая компонента кривой $C^{i}$, стягиваемая в категории $X$; тогда кривая $\left(\sigma_{i}\right)_{*} B^{i}$ также стягиваема в категории $X$. Для доказательства этого утверждения нужно рассмотреть два случая: $n_{i}>2$ и $n_{i}=2$, поскольку случаи $n_{i}=1$ и $n_{i}=0$ очевидны.

Итак, пусть $n_{i}>2, B^{i}$ - неприводимая компонента кривой $C^{i}$. Если $\left(B^{i}, E^{i}\right)=0$, то очевидно, что кривая $B^{i}$ стягиваема в категории $X$ тогда и только тогда, когда кривая $\left(\sigma_{i}\right)_{*} B^{i}$ стягиваема в $X$. Если $\left(B^{i}, E^{i}\right)>0$, то кривая $B^{i}$ не может быть стягиваема в $X$ согласно теореме 5.12. Действительно, предположим, что $B^{i}$ стягиваема в категории $X$. Из связности $C$, того, что $n_{i}>2,\left(E^{i}, C^{i}-E^{i}\right)=1$, и теоремы 5.12 следует, что $\left(B^{i}, C^{i}-B^{i}\right)=2$. Тогда из $(V, C) \in \mathrm{Ob}^{M} R^{N}$ вытекает, что либо $\left(B^{i}\right)^{2}=\left(B_{i}^{V}\right)^{2} \neq-1$, либо на $B^{i}$ должны лежать особые точки пары $\left(V^{i}, C^{i}\right)$ типов $0_{n_{i}^{j}, 1, k_{i}^{j}}$, где $k_{i}^{j} \leqslant 2$. Это противоречит стягиваемости кривой $B^{i}$ в категории $X$. Таким образом, наше утверждение в случае $n_{i}>2$ доказано. 
Осталось рассмотреть случай $n_{i}=2$, когда $C^{i}=E^{i}+B^{i},\left(E^{i}, B^{i}\right)=1$. Допустим, что кривая $B^{i}$ стягиваема в категории $X$, а $\left(\sigma_{i}\right)_{*} B^{i}$ не стягиваема в $X$. Из стягиваемости $B^{i}$ и теоремы 5.12 следует, что $\left(B_{i}^{V}\right)^{2} \leqslant\left(B^{i}\right)^{2}<0$ и на $B^{i}$ лежит не более одной особой точки пары $\left(V^{i}, C^{i}\right)$ типа $0_{n_{i}, 1, k_{i}}$, где $k_{i} \leqslant 2$, поэтому $\left(B_{i}^{V}, C-B_{i}^{V}\right) \leqslant 2$. Поскольку кривая $\left(\sigma_{i}\right)_{*} B^{i}$ не стягиваема в $X$, из леммы 4.4 вытекает, что $\left(\left(\sigma_{i}\right)_{*} B^{i}\right)^{2} \geqslant 0$. Поэтому в данной ситуации можно применить лемму 6.1 , согласно которой $\left(B_{i}^{V}\right)^{2}=-1,\left(B_{i}^{V}, C-B_{i}^{V}\right)=2$. Это противоречит минимальности пары $(V, C)$ в категории $R^{N}$. Таким образом, вспомогательное утверждение доказано.

Следовательно, $\operatorname{Ex}(\sigma)=\operatorname{Ex}(\psi)$. Тогда из леммы 4.4 вытекает, что множество $M_{X}^{\vee}(V, C)$ состоит из одного класса изоморфных объектов категории $B_{1}\left(X^{\vee}\right)$. Очевидно, что $\left(\left(Y, C^{Y}\right), \sigma^{\vee}\right) \in M_{X}^{\vee}(V, C)$ тогда и только тогда, когда $((V, C), \sigma) \in N_{X}\left(Y, C^{Y}\right)$. Теорема доказана.

ЗАмЕчАнИЕ 6.3 . В категории $R_{1}$ утверждение, аналогичное теореме 6.2 , не выполняется.

ПримеР 6.4. Пусть $V$ - неособая поверхность, $C=\sum_{i=1}^{4} C_{i}$ - кривая типа $0_{4,0,2}$ на $V,\left(C_{1}\right)^{2}=\left(C_{4}\right)^{2}=-2,\left(C_{2}\right)^{2}=\left(C_{3}\right)^{2}=-1$. Тогда очевидно, что $(V, C) \in \mathrm{Ob}^{M} R_{1}^{N}$ и $\left(\left(V^{1}, C^{1}\right), \varphi_{1}\right),\left(\left(V^{2}, C^{2}\right), \varphi_{2}\right) \in M_{R_{1}}(V, C)$, где $\varphi_{1}-$ стягивание кривых $C_{1}, C_{2}$ и $C_{4}$, а $\varphi_{2}$ - стягивание $C_{1}, C_{3}$ и $C_{4}$. Заметим, что $\operatorname{Ex}\left(\varphi_{1}\right) \neq \operatorname{Ex}\left(\varphi_{2}\right)$ и пара $(V, C)$ может быть выбрана таким образом, чтобы $\left(\left(V^{1}, C^{1}\right), \varphi_{1}^{\vee}\right)$ и $\left(\left(V^{2}, C^{2}\right), \varphi_{2}^{\vee}\right)$ принадлежали различным классам изоморфных объектов категории $B_{1}\left(R_{1}^{\vee}\right)$.

ЗАмечАниЕ 6.5. Пусть $X$ - одна из категорий $R_{p_{\#}}$ при $p<1 / 2$. Тогда в категории $X$ утверждение, аналогичное теореме 6.2 , не верно.

Пример 6.6. Пусть $V$ - неособая поверхность, $C=\sum_{i=1}^{3} C_{i}+\sum_{i=1}^{3} C_{i}^{\prime}-$ кривая на $V$ такая, что $C_{i}, C_{i}^{\prime}$ неособы, рациональны и $\left(C_{i}\right)^{2}=\left(C_{i}^{\prime}\right)^{2}=-1$, $\left(C_{i}, C_{j}\right)=\left(C_{i}^{\prime}, C_{j}^{\prime}\right)=0$ при $i \neq j,\left(C_{i}, C_{j}^{\prime}\right)=1$ при любых $i, j$. Тогда очевидно, что $(V, C) \in \mathrm{Ob}^{M} X^{N}$ и $\left(\left(V^{1}, C^{1}\right), \varphi_{1}\right),\left(\left(V^{2}, C^{2}\right), \varphi_{2}\right) \in M_{X}(V, C)$, где $\varphi_{1}$ - стягивание всех кривых $C_{i}$, а $\varphi_{2}$ - стягивание всех $C_{i}^{\prime}$. Заметим, что $\operatorname{Ex}\left(\varphi_{1}\right) \neq \operatorname{Ex}\left(\varphi_{2}\right)$ и пара $(V, C)$ может быть выбрана таким образом, чтобы $\left(\left(V^{1}, C^{1}\right), \varphi_{1}^{\vee}\right)$ и $\left(\left(V^{2}, C^{2}\right), \varphi_{2}^{\vee}\right)$ принадлежали различным классам изоморфных объектов категории $B_{1}\left(X^{\vee}\right)$.

Пусть $X$ - одна из категорий $R, R_{p_{\#}}$ при $p<1$ или $R_{1_{-}}$.

ОПРЕДЕЛЕНИЕ 6.7. 1) Введем функтор разрешения особенностей $\mathcal{N}_{X}: X \rightarrow$ $R^{N} \sim X^{N}$ следующим образом. Начнем с определения $\mathcal{N}_{X}$ на объектах категории $X$. Согласно утверждению $4.7,2), 3)$ для $(V, C) \in \mathrm{Ob} X$ множество $N_{X}(V, C)=N_{R}(V, C)$ состоит из одного класса изоморфных объектов категории $B_{1}(X)$. Выберем по одному элементу $\left(\left(W, C^{W}\right), \pi\right)$ из каждого такого класса. (Здесь и далее выбранные морфизмы будут обозначаться через $\pi, \pi^{\prime}$.) Положим

$$
\mathcal{N}_{X}(V, C):=\left(W, C^{W}\right)
$$

Теперь определим функтор $\mathcal{N}_{X}$ на морфизмах $X$. Пусть $\psi:(V, C) \rightarrow\left(V^{\prime}, C^{\prime}\right)$, $\psi \in \operatorname{Mor} X$ и $\left(\left(W, C^{W}\right), \pi\right) \in N_{X}(V, C),\left(\left(W^{\prime}, C^{W^{\prime}}\right), \pi^{\prime}\right) \in N_{X}\left(V^{\prime}, C^{\prime}\right)$. 
Поскольку $\left(\left(W, C^{W}\right), \psi \circ \pi\right) \in \mathrm{Ob} B_{1}\left(R, R^{N},\left(V^{\prime}, C^{\prime}\right)\right)$, из утверждения $\left.4.7,2\right)$ следует, что определен морфизм $\mathcal{N}_{X}(\psi) \in \operatorname{Mor} R^{N}$ :

$$
\mathcal{N}_{X}(\psi):=\left(\pi^{\prime}\right)^{-1} \circ \psi \circ \pi:\left(W, C^{W}\right) \rightarrow\left(W^{\prime}, C^{W^{\prime}}\right) .
$$

Очевидно, что определенный выше функтор $\mathcal{N}_{X}$ удовлетворяет условию композиции морфизмов и этот функтор является строгим.

2) Функтор $\mathcal{N}_{X}$ индуцирует функтор $\widetilde{\mathcal{N}}_{X}: \widetilde{X} \rightarrow \widetilde{R}^{N}$, определяемый следующим образом: если $(V, C) \in \mathrm{Ob} \widetilde{X}=\mathrm{Ob} X$, положим $\widetilde{\mathcal{N}}_{X}(V, C):=\mathcal{N}_{X}(V, C)$.

Для определения функтора $\widetilde{\mathcal{N}}_{X}$ на Mor $\widetilde{X}$ продолжим сначала функтор $\mathcal{N}_{X}$ на объекты категории $B_{2}(X)$ :

$$
\mathcal{N}_{X}\left(\left(W, C^{W}\right), \varphi, \varphi^{\prime}\right):=\left(\mathcal{N}_{X}\left(W, C^{W}\right), \mathcal{N}_{X}(\varphi), \mathcal{N}_{X}\left(\varphi^{\prime}\right)\right) .
$$

Легко видеть, что если $\left(\left(W, C^{W}\right), \varphi, \varphi^{\prime}\right)^{B_{2}(X)}\left(\left(W^{\prime}, C^{W^{\prime}}\right), \psi, \psi^{\prime}\right)$, то

$$
\mathcal{N}_{X}\left(\left(W, C^{W}\right), \varphi, \varphi^{\prime}\right)^{B_{2}\left(R^{N}\right)} \mathcal{N}_{X}\left(\left(W^{\prime}, C^{W^{\prime}}\right), \psi, \psi^{\prime}\right)
$$

Поэтому функтор $\widetilde{\mathcal{N}}_{X}$ можно корректно определить на множестве Mor $\widetilde{X}$. Его согласованность с композицией морфизмов очевидна.

ОПРЕДЕЛЕНИЕ 6.8. Функтором минимизации $\widetilde{\mathcal{M}}_{X}: \widetilde{R}^{N M} \rightarrow \widetilde{X}^{M}$, где $X-$ одна из категорий $R_{p_{\#}}$ при $4 / 5<p<1$ или $R_{1_{-}}$, а $\widetilde{R}^{N M}=\widetilde{R}^{N}\left(\mathrm{Ob}^{M} R^{N}\right)$, называется функтор, заданный следующим образом. Пусть $(V, C) \in \mathrm{Ob}^{M} R^{N}$ и $\left(\left(Y, C^{Y}\right), \pi\right) \in M_{X}(V, C)$ (или, что равносильно, $(V, C) \in N_{X}\left(Y, C^{Y}\right)$, где $\pi$ выбранный морфизм). Положим

$$
\widetilde{\mathcal{M}}_{X}(V, C):=\left(Y, C^{Y}\right) .
$$

Для определения функтора $\widetilde{\mathcal{M}}_{X}$ на морфизмах Mor $\widetilde{R}^{N M}$ определим $\widetilde{\mathcal{M}}_{X}$ на $\mathrm{Ob} B_{2}\left(R^{N}, R^{N}, \mathrm{Ob}^{M} R^{N}\right)$ следующим образом. Пусть

$$
\begin{gathered}
\left(\left(W, C^{W}\right), \varphi, \varphi^{\prime}\right) \in \mathrm{Ob} B_{2}\left(R^{N}, R^{N}, \mathrm{Ob}^{M} R^{N}\right), \\
\varphi:\left(W, C^{W}\right) \rightarrow(V, C), \quad\left(\left(Y, C^{Y}\right), \pi\right) \in M_{X}(V, C), \\
\varphi^{\prime}:\left(W, C^{W}\right) \rightarrow\left(V^{\prime}, C^{\prime}\right), \quad\left(\left(Y^{\prime}, C^{Y^{\prime}}\right), \pi^{\prime}\right) \in M_{X}\left(V^{\prime}, C^{\prime}\right) .
\end{gathered}
$$

Положим

$$
\widetilde{\mathcal{M}}_{X}\left(\left(W, C^{W}\right), \varphi, \varphi^{\prime}\right):=\left(\left(W, C^{W}\right), \pi \circ \varphi, \pi^{\prime} \circ \varphi^{\prime}\right)
$$

Очевидно, что если $\left(\left(W, C^{W}\right), \varphi, \varphi^{\prime}\right)^{B_{2}\left(R^{N}\right)}\left(\left(W^{\prime}, C^{W^{\prime}}\right), \psi, \psi^{\prime}\right)$, то

$$
\widetilde{\mathcal{M}}_{X}\left(\left(W, C^{W}\right), \varphi, \varphi^{\prime}\right)^{B_{2}(X)} \widetilde{\mathcal{M}}_{X}\left(\left(W^{\prime}, C^{W^{\prime}}\right), \psi, \psi^{\prime}\right) .
$$

Поэтому функтор $\widetilde{\mathcal{M}}_{X}$ определен на множестве Mor $\widetilde{R}^{N M}$. Очевидно также, что $\widetilde{\mathcal{M}}_{X}\left(\gamma_{1} \circ \gamma_{2}\right)=\widetilde{\mathcal{M}}_{X}\left(\gamma_{1}\right) \circ \widetilde{\mathcal{M}}_{X}\left(\gamma_{2}\right)$, где $\gamma_{1}, \gamma_{2} \in \operatorname{Mor} \widetilde{R}^{N M}$. Таким образом, функтор $\widetilde{\mathcal{M}}_{X}$ корректно определен.

Пусть $\widetilde{X}_{N}^{M}$ - образ категории $\widetilde{R}^{N M}$ относительно функтора минимизации. 
Теорема 6.9. Функтор $\widetilde{\mathcal{M}}_{X}$ является строгим и полным, поэтому он задает вложение категории $\widetilde{R}^{N M}$ в $\widetilde{X}^{M}$ в качестве полной подкатегории $\widetilde{X}_{N}^{M} \sim \widetilde{R}^{N M}$. Ограничение функтора $\widetilde{\mathcal{N}}_{X}$ на $\widetilde{X}_{N}^{M}$ является функтором, обратным $\kappa \widetilde{\mathcal{M}}_{X}$.

ДокАЗАТЕЛЬСтво. Если $\left(\left(W, C^{W}\right), \varphi, \varphi^{\prime}\right) \in \mathrm{Ob} B_{2}\left(R^{N}, R^{N}, \mathrm{Ob}^{M} R^{N}\right)$, то

$$
\mathcal{N}_{X}\left(\widetilde{\mathcal{M}}_{X}\left(\left(W, C^{W}\right), \varphi, \varphi^{\prime}\right)\right)=\left(\left(W, C^{W}\right), \varphi, \varphi^{\prime}\right)
$$

Теперь согласно определению $6.7,2)$ если $\left(\left(Y, C^{Y}\right), \rho, \rho^{\prime}\right) \stackrel{B_{2}(X)}{=}\left(\left(Y^{\prime}, C^{Y^{\prime}}\right), \psi, \psi^{\prime}\right)$, то имеем

$$
\mathcal{N}_{X}\left(\left(Y, C^{Y}\right), \rho, \rho^{\prime}\right)^{B_{2}\left(R^{N}\right)} \mathcal{N}_{X}\left(\left(Y^{\prime}, C^{Y^{\prime}}\right), \psi, \psi^{\prime}\right)
$$

Отсюда следует строгость функтора $\widetilde{\mathcal{M}}_{X}$. Его полнота, а также то, что ограничение $\widetilde{\mathcal{N}}_{X}$ на $\widetilde{X}_{N}^{M}$ является функтором, обратным к $\widetilde{\mathcal{M}}_{X}$, вытекает из того, что если $\left(\left(W, C^{W}\right), \varphi, \varphi^{\prime}\right) \in \mathrm{Ob} B_{2}\left(X, X, \mathrm{Ob} \widetilde{X}_{N}^{M}\right)$, то

$$
\widetilde{\mathcal{M}}_{X}\left(\mathcal{N}_{X}\left(\left(W, C^{W}\right), \varphi, \varphi^{\prime}\right)\right) \stackrel{B_{2}(X)}{\underline{(}}\left(\left(W, C^{W}\right), \varphi, \varphi^{\prime}\right) .
$$

Теорема доказана.

ЗАмечАниЕ 6.10 . Категория $\widetilde{X}_{N}^{M}$ не совпадает с $\widetilde{X}^{M}$, поскольку существуют пары $(V, C) \in \mathrm{Ob}^{M} T$ такие, что $\mathcal{N}_{X}(V, C)=\left(W, C^{W}\right) \notin \mathrm{Ob}^{M} R^{N}$.

Пример 6.11. Пусть $W$ - неособая поверхность, $C^{W}=\sum_{i=1}^{3} C_{i}-$ кривая типа $0_{3,0,2}$ на $W,\left(C_{1}\right)^{2}=\left(C_{3}\right)^{2}=-2,\left(C_{2}\right)^{2}=-1$. Тогда $\left(W, C^{W}\right) \notin \mathrm{Ob}^{M} R^{N}$, a $(V, C) \in \mathrm{Ob}^{M} X$, где $(V, C)$ получается из $\left(W, C^{W}\right)$ стягиванием кривых $C_{1}$ и $C_{3}$, поскольку $C$ - неприводимая кривая и $C^{2}=0$.

Лемма 6.12. Пусть $\left(\left(Z, C^{Z}\right), \psi, \psi^{\prime}\right) \in \mathrm{Ob}^{M} B_{2}\left(X, X, \mathrm{Ob} \widetilde{X}_{N}^{M}\right) ;$ тогда имеем $\mathcal{N}_{X}\left(\left(Z, C^{Z}\right), \psi, \psi^{\prime}\right) \in \mathrm{Ob}^{M} B_{2}\left(R^{N}, R^{N}, \mathrm{Ob}^{M} R^{N}\right)$. В частности, если выполнено $\gamma \in \operatorname{Mor} \widetilde{R}^{N M}$ u $\gamma_{M}=\widetilde{\mathcal{M}}_{X}(\gamma), \operatorname{mo} L_{R^{N}}(\gamma)=\mathcal{N}_{X}\left(L_{X}\left(\gamma_{M}\right)\right)$.

ДокАЗАтеЛЬСтво. Пусть $\left(\left(W, C^{W}\right), \varphi, \varphi^{\prime}\right)=\mathcal{N}_{X}\left(\left(Z, C^{Z}\right), \psi, \psi^{\prime}\right)$ и условия леммы представлены коммутативной диаграммой

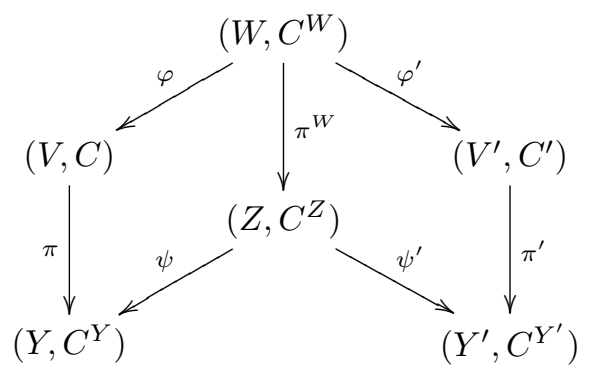

Допустим, что доказываемое утверждение не выполнено. Тогда на $\left(W, C^{W}\right)$ существует кривая $E$, стягиваемая в категории $R^{N}$ относительно морфизмов $\varphi$ и $\varphi^{\prime}$ (т. е. существует морфизм $\varepsilon \in \operatorname{Mor} B_{2}\left(R^{N}, R^{N}, \mathrm{Ob}^{M} R^{N}\right), \varepsilon:\left(\left(W, C^{W}\right)\right.$, $\left.\varphi, \varphi^{\prime}\right) \rightarrow\left(\left(T, C^{T}\right), \rho, \rho^{\prime}\right)$, стягивающий $E$ на $\left.W\right)$. Из определения функтора $\mathcal{N}_{X}$ на объектах категории $B_{2}(X)$ следует, что образом кривой $E$ на $Z$ должна 
быть кривая $E^{Z}$, не стягиваемая в категории $X$. С другой стороны, из коммутативности диаграммы (6.1) вытекает, что кривая $E^{Z}$ должна стягиваться морфизмами $\psi$ и $\psi^{\prime}$. Поэтому $\left(E^{Z}\right)^{2}<0$ и $E^{Z}$ стягиваема в категории $X$ согласно лемме 4.4. Противоречие. Лемма доказана.

Tеорема 6.13. Множество Оb $\widetilde{X}_{N}^{M}$, где $X$ - одна из категорий $R_{p_{\#}}$ при $4 / 5<p<1$ или $R_{1_{-}}$, замкнуто в $\mathrm{Ob}^{M} X$ (см. определение 2.19).

ДокАзАтельство. Допустим, что утверждение теоремы не выполнено. Тогда существуют

$$
\left(Y, C^{Y}\right) \in \mathrm{Ob}^{M} X, \quad \psi \in \operatorname{Mor} X, \quad\left(\left(Z, C^{Z}\right), \psi_{1}, \psi_{2}\right) \in \mathrm{Ob}^{M} B_{2}\left(X, X, \mathrm{Ob} \widetilde{X}_{N}^{M}\right)
$$

такие, что $\left(\left(Y, C^{Y}\right), \psi\right) \in M_{X}\left(Z, C^{Z}\right)$ и $(V, C)=\mathcal{N}_{X}\left(Y, C^{Y}\right) \notin \mathrm{Ob}^{M} R^{N}$. Поэтому на $V$ существует кривая $E$, стягиваемая в категории $R^{N}$ и такая, что кривая $E^{Y}=\pi_{*} E$ не стягиваема в категории $X$, где $((V, C), \pi) \in N_{X}\left(Y, C^{Y}\right)$. Тогда из лемм $6.1,4.4$ следует, что $(E)^{2}=-1,(E, C-E)=2,\left(E^{Y}, C^{Y}-E^{Y}\right)=0$ и на $E^{Y}$ лежат две особые точки пары $\left(Y, C^{Y}\right)$ типов $0_{n_{1}, 1, k_{1}}$ и $0_{n_{2}, 1, k_{2}}$, где $k_{1}, k_{2} \leqslant 2$. Пусть $\left(\left(W, C^{W}\right), \varphi, \varphi^{\prime}\right)=\mathcal{N}_{X}\left(\left(Z, C^{Z}\right), \psi_{1}, \psi_{2}\right)$, диаграмма

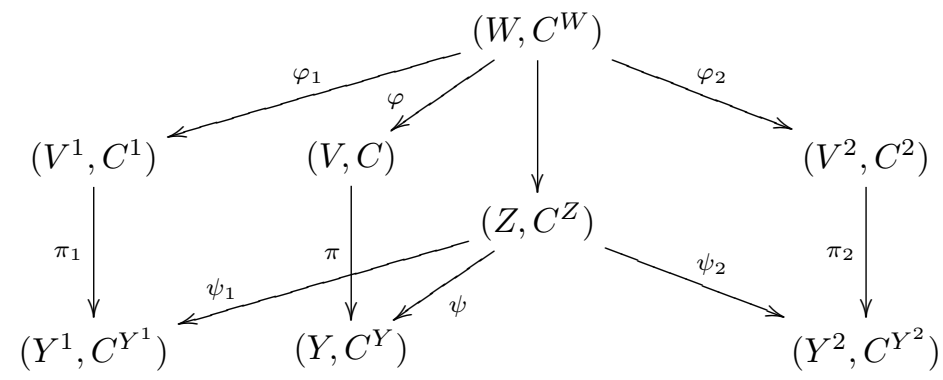

коммутативна и $E^{W}=\varphi^{-1}(E), E_{0}^{W}=\varphi_{\text {соб }}^{-1}(E)$.

Очевидно, что кривая $E^{W}$ стягиваема в категории $R^{N}$. Мы докажем, что $E^{W}$ должна стягиваться морфизмами $\varphi_{1}$ и $\varphi_{2}$. Таким образом, согласно лемме 4.4 будет получено противоречие с тем, что $\left(\left(W, C^{W}\right), \varphi_{1}, \varphi_{2}\right) \in$ $\mathrm{Ob}^{M} B_{2}\left(R^{N}, R^{N}, \mathrm{Ob}^{M} R^{N}\right)$ по лемме 6.12.

Поскольку все рассуждения локальны, можно считать, что кривые $C^{Y}, C$, $C^{W}$ связны, и тогда кривая $C^{Y}$ состоит из одной компоненты $E^{Y}$. Допустим, что кривая $E^{W}$ не стягивается морфизмом $\varphi_{1}$. Тогда, если кривая $E_{0}^{W}$ также не стягивается $\varphi_{1}$, согласно тому, что множество минимальных разрешений особенностей любого объекта категории $X$ состоит из одного класса изоморфных объектов категории $B_{1}(X)$, и ввиду стягиваемости кривой $E^{W}-E_{0}^{W}$ в этой категории получаем, что $\left(E^{1}\right)^{2}=-1,\left(E^{1}, C^{1}-E^{1}\right)=2$, где $E^{1}=\left(\varphi_{1}\right)_{*} E_{0}^{W}$. Таким образом, получаем противоречие с тем, что $\left(V^{1}, C^{1}\right) \in \mathrm{Ob}^{M} R^{N}$. Поэтому кривая $E_{0}^{W}$ должна стягиваться морфизмами $\varphi_{1}$ и $\varphi_{2}$.

Допустим теперь, что морфизм $\varphi_{1}$ стягивает кривую $E_{0}^{W}$ и не стягивает $E^{W}$. Пусть $\varphi_{1}=\sigma_{1} \circ \cdots \circ \sigma_{n}, \sigma_{i} \in \operatorname{Mor}^{1} R^{N}, \sigma_{i}:\left(W^{i}, C^{W^{i}}\right) \rightarrow\left(W^{i-1}, C^{W^{i-1}}\right)$, $\left(W^{n}, C^{W^{n}}\right)=\left(W, C^{W}\right),\left(W^{0}, C^{W^{0}}\right)=\left(V^{1}, C^{1}\right), E^{n}=E^{W}, E_{0}^{n}=E_{0}^{W}, E^{i-1}=$ $\left(\sigma_{i}\right)_{*} E^{i}, E_{0}^{i-1}=\left(\sigma_{i}\right)_{*} E_{0}^{i}$, и пусть $\sigma_{k}$ стягивает кривую $E_{0}^{k}$. Заметим, что $\rho \in \operatorname{Mor} X$, где $\rho:\left(W^{k}, C^{W^{k}}\right) \rightarrow\left(Y, C^{Y}\right)$ - стягивание кривой $E^{k}-E_{0}^{k}$, так как морфизмы $\sigma_{j}, j>k$, не стягивали собственные прообразы компонент 
кривой $C^{Y}=E^{Y}$. Тогда из того, что множество минимальных разрешений особенностей любого объекта категории $X$ состоит из одного класса изоморфных объектов категории $B_{1}(X)$, вытекает, что существует $\varepsilon \in \operatorname{Mor} R^{N}$, $\varepsilon:\left(W^{k}, C^{W^{k}}\right) \rightarrow(V, C), \varepsilon_{\text {соб }}^{-1}(E)=E_{0}^{k}, \varepsilon^{-1}(E)=E^{k}$, и $E^{k} \neq E_{0}^{k}$. Тогда из следствия $3.5,1)$ вытекает, что $\left(E_{0}^{k}\right)^{2}<(E)^{2}=-1$, и мы получаем противоречие с тем, что $\sigma_{k} \in$ Mor $R^{N}$. Теорема доказана.

ЗАмечАниЕ 6.14. Пусть $\Theta$ - некоторое подмножество в $\mathrm{Ob}^{M} R^{N}$. Тогда из теоремы 6.13 и леммы 6.12 следует, что $\Theta$ замкнуто в $\mathrm{Ob}^{M} R^{N}$, если и только если $\mathcal{M}_{X}(\Theta)$ замкнуто в $\mathrm{Ob}^{M} X$, где $X$ - одна из категорий $R_{p_{\#}}$ при $4 / 5<p<1$ или $R_{1-}$.

\section{§ 7. Определение кривых $F_{X}(V, C)$, их классификация и инвариантность относительно функтора разрешения особенностей}

ОПРЕДЕЛЕНИЕ 7.1. Пусть $X$ - одна из категорий $R_{p_{\#}}$ или $R^{N}$ и $(V, C) \in$ $\mathrm{Ob} X$. Введем кривую $F_{X}(V, C)$ следующим образом:

$$
F_{X}(V, C):=\sum_{i \in I} E_{i}
$$

где $E_{i}$ - неприводимые кривые на $V$ и $i \in I$, если существует объект $\left(\left(W_{i}, C^{W_{i}}\right)\right.$, $\left.\varphi_{i}, \varphi_{i}^{\prime}\right)$ категории $B_{2}(X)$, где $\varphi_{i}:\left(W_{i}, C^{W_{i}}\right) \rightarrow(V, C), \varphi_{i}^{\prime}:\left(W_{i}, C^{W_{i}}\right) \rightarrow\left(V_{i}^{\prime}, C_{i}^{\prime}\right)$, такой, что морфизм $\varphi_{i}^{\prime}$ стягивает кривую $\left(\varphi_{i}\right)_{\text {соб }}^{-1}\left(E_{i}\right)$.

ЗАмечание 7.2. Пусть $X$ такая, как в определении 7.1, и $(V, C) \in \operatorname{Ob} X$. Тогда:

1) для пары $(V, C) \in \mathrm{Ob}^{X}$ равенство нулю дивизора $F_{X}(V, C)$ является критерием ее правожесткости и минимальности;

$2)$ если категория $Y$ является подкатегорией в категории $X$ и $(V, C) \in \operatorname{Ob} Y$, то $F_{Y}(V, C) \subset F_{X}(V, C)$;

$3)$ в определении 7.1 можно заменить условие $\left(\left(W, C^{W}\right), \varphi, \varphi^{\prime}\right) \in \mathrm{Ob} B_{2}(X)$ на условие $\left(\left(W, C^{W}\right), \varphi, \varphi^{\prime}\right) \in \mathrm{Ob}^{M} B_{2}(X)$ (а также на условие $\left(\left(W, C^{W}\right), \varphi, \varphi^{\prime}\right) \in$ $\mathrm{Ob}^{M} B_{2}\left(X, X, \mathrm{Ob}^{M} X\right)$, если $\left.(V, C) \in \mathrm{Ob}^{M} X\right)$.

УтВеРЖДЕНИЕ 7.3. Пусть $X-$ одна из категорий $R_{p_{\#}}$ или $R^{N},\left(\left(W, C^{W}\right)\right.$, $\left.\varphi, \varphi^{\prime}\right) \in \mathrm{Ob}^{M} B_{2}\left(X, X, \mathrm{Ob}^{M} X\right), \varphi:\left(W, C^{W}\right) \rightarrow(V, C), \varphi^{\prime}:\left(W, C^{W}\right) \rightarrow\left(V^{\prime}, C^{\prime}\right)$ и $F$ - кривая на $V$ такая, что морфизм $\varphi^{\prime}$ является стягиванием кривой $D$, $\varphi_{*}(D)=F\left(\right.$ очевидно, что $\left.F \subset F_{X}(V, C)\right)$. Тогда $\varphi(\operatorname{Ex}(\varphi)) \subset F$.

ДоказАтельство. Допустим, что $\varphi(\operatorname{Ex}(\varphi)) \not \subset F$. Из того, что $\left(\left(W, C^{W}\right)\right.$, $\left.\varphi, \varphi^{\prime}\right) \in \mathrm{Ob}^{M} B_{2}\left(X, X, \mathrm{Ob}^{M} X\right)$, следует, что существует точка $x \in V \backslash F$ такая, что $E=\varphi^{-1}(x)$ - кривая на $W$, не стягиваемая морфизмом $\varphi^{\prime}$. С другой стороны, из леммы 4.4 вытекает, что кривая $\varphi_{*}^{\prime}(E)$ стягиваема в категории $X$, что противоречит минимальности пары $\left(V^{\prime}, C^{\prime}\right)$. Утверждение доказано.

ЗАмечАниЕ 7.4. 1) Пусть $X$ - одна из категорий $R^{N}, R_{p_{\#}}$ при $4 / 5<p<1$ или $R_{1_{-}}, \varphi \in \operatorname{Mor} X, \varphi:\left(W, C^{W}\right) \rightarrow(V, C), F=F_{X}(V, C), F^{W}=F_{X}\left(W, C^{W}\right)$, 
$E_{i}, i \in\{1, \ldots, n\},-$ все $\varphi$-исключительные кривые на $W$. Тогда из выполненности левого правила Оре в категории $X$ следует, что

$$
F^{W}=\left(\varphi_{\text {coб }}^{-1}(F)+\sum_{i=1}^{n} E_{i}\right) .
$$

2) Для категории $R_{1}$ утверждение типа 1) не выполнено.

Пример 7.5. Пусть $V$ - неособая поверхность, $C$ - неприводимая неособая кривая на $V$ с $C^{2}=1, \varphi:\left(W, C^{W}\right) \rightarrow(V, C)$ - раздутие точки $x$ на кривой $C, E=\operatorname{Ex}(\varphi), C^{W}=\varphi_{\text {соб }}^{-1}(C)+E$. Тогда очевидно, что $F_{R_{1}}(V, C)=C$. С другой стороны, используя теорему 5.12 , несложно убедиться в том, что $F_{R_{1}}\left(W, C^{W}\right)=E \neq C^{W}$, поскольку не существует такого $\left(\left(W, C^{W}\right), \pi, \pi^{\prime}\right) \in$ $\mathrm{Ob} B_{2}\left(R_{1}\right), \pi:\left(W, C^{W}\right) \rightarrow\left(V^{\prime}, C^{\prime}\right)$, что $\pi^{\prime}$ стягивает кривую $(\varphi \circ \pi)_{\text {соб }}^{-1}(C)$.

Лемма 7.6. Пусть $X$ - одна из категорий $R_{p_{\#}}$ при $p>4 / 5$ или $R^{N}$, $\left(\left(W, C^{W}\right), \varphi, \varphi^{\prime}\right) \in \mathrm{Ob} B_{2}(X), \varphi:\left(W, C^{W}\right) \rightarrow(V, C), \varphi^{\prime}:\left(W, C^{W}\right) \rightarrow\left(V^{\prime}, C^{\prime}\right)$, $(V, C) \in \mathrm{Ob}^{M} X, E \subset C$ - неприводимая кривая на $V$ такая, что кривая $E^{W}=\varphi_{\text {соб }}^{-1}$ стягивается морфизмом $\varphi^{\prime}$. Тогда $p_{g}(E)=0$ и для кривой $E$ имеем:

1) если $X=R_{1}$, то $(E, C-E)=0, p_{a}(E)=0$ и $E$ содержит не более двух особых точек пары $(V, C)$ типов $0_{n_{1}, 1, k_{1}} u 0_{n_{2}, 1, k_{2}}$, где $k_{i} \leqslant 2$ (cм. определение 5.2);

2) если $X=R_{p_{\#}}$ при $4 / 5<p<1$ или $X=R_{1_{-}}$, то:

a) либо $E$ такая, как в случае категории $R_{1}$;

b) либо $(E, C-E)=0, E$ не содержит особенностей пары $(V, C), p_{a}(E)=1$ и кривая $E$ имеет ровно одну обыкновенную двойную точку (заметим, что в этом случае $E$ имеет тип $1_{1,0,1}$ в $\left.(V, C)\right)$;

c) либо $(E, C-E)=1, p_{a}(E)=0$ и $E$ содержит не более одной особой точки nары $(V, C)$ muna $0_{n, 1, k}$, где $k \leqslant 2$;

d) либо $(E, C-E)=2, E$ не содержит особенностей парь $(V, C)$ и $p_{a}(E)=0$;

3) если $X=R^{N}$, то либо $p_{a}(E)=0 u(E, C-E) \leqslant 2$, либо $(E, C-E)=0$, $p_{a}(E)=1$ и $E$ содержит только одну обыкновенную двойную точку.

ДокАЗАТЕЛЬСтво. Пусть $\varphi^{\prime}=\sigma_{1} \circ \cdots \circ \sigma_{n}$ - разложение морфизма $\varphi$, где $\sigma_{i}:\left(V^{i}, C^{i}\right) \rightarrow\left(V^{i-1}, C^{i-1}\right), \sigma_{i} \in \operatorname{Mor}^{1} X,\left(V^{0}, C^{0}\right)=\left(V^{\prime}, C^{\prime}\right),\left(V^{n}, C^{n}\right)=$ $\left(W, C^{W}\right)$, и пусть морфизм $\sigma_{k}$ стягивает кривую $E^{k}=\left(\sigma_{k+1} \circ \cdots \circ \sigma_{n}\right)_{*} E^{W}$. Тогда $\left(E^{k}, C^{k}-E^{k}\right)>0$, поскольку в противном случае из леммы 4.4 следовало бы, что кривая $E$ стягиваема в категории $X$, а это противоречит минимальности пары $(V, C)$.

Заметим, что если $B$ - компонента кривой $C$, то из того, что $(V, C) \in \mathrm{Ob}^{M} X$, следует, что $B^{k}=\left(\sigma_{k+1} \circ \cdots \circ \sigma_{n} \circ \varphi^{-1}(B)\right)$ - кривая на $V^{k}$, и если $(B, E)>0$, то $\left(B^{k}, E^{k}\right)>0$. Заметим также, что если $x$ - особая точка пары $(V, C)$ типа $0_{n, 1, k}$, где $k \leqslant 2$, лежащая на $E$, то либо $H^{k}:=\left(\sigma_{k+1} \circ \cdots \circ \sigma_{n} \circ \varphi^{-1}(x)\right)-$ кривая на $V^{k}$ такая, что $\left(H^{k}, E^{k}\right)=1$, либо $\left(\sigma_{k+1} \circ \cdots \circ \sigma_{n} \circ \varphi^{-1}(x)\right)$ - особая точка пары $\left(V^{k}, C^{k}\right)$ типа $0_{n, 1, k}$, лежащая на $E^{k}$.

Если бы индекс пересечения $(E, C-E)$ был больше, чем указано в условии леммы, или если бы $E$ содержала больше особых точек пары $(V, C)$ либо собственных особенностей, чем указано в этом условии, то кривая $E^{k}$ не удовлетворяла бы условиям теоремы 5.12 или замечания 4.2 и была не стягиваема 
в категории $X$. (Заметим, что если кривая $E$ имеет обыкновенную двойную точку, то $\left(E^{k}, C^{k}-E^{k}\right) \geqslant 2$. Отсюда следует, что $E$ не содержит особенностей пары $(V, C),(E, C-E)=0$, и $E$ не может иметь других особых точек.) Лемма доказана.

Лемма 7.7. Пусть $X$ - одна из категорий $R_{p_{\#}}$ при $p>4 / 5$ или $R^{N}$, $\left(\left(W, C^{W}\right), \varphi, \varphi^{\prime}\right) \in \mathrm{Ob}^{M} B_{2}(X), \varphi:\left(W, C^{W}\right) \rightarrow(V, C),(V, C) \in \mathrm{Ob}^{M} X u$ $F \neq 0$ - кривая на $V$ такая, что морфизм $\varphi^{\prime}$ является стягиванием кривой $D$, $\varphi_{*}(D)=F$. Тогда любая максимальная связная компонента $B=\sum_{i=1}^{n} B_{i}$ кривой $F$ (т.е. связная компонента, не содержащаяся ни в какой связной компоненте $F$, отличной от нее самой) содержит неприводимую компоненту с неотрищательным индексом самопересечения, и если кривая $B$ имеет mun $1_{1,0,1}$ в $(V, C)$, mo $B^{2} \geqslant 3$.

ДокАЗАТЕЛЬСтво. Из леммы 7.6 следует, что кривая $B$ имеет тип $0_{n, m, k}$ или $1_{n, 0, k}$ в $(V, C)$, где $m, k \leqslant 2$; в последнем случае она является колесом (см. замечание $5.3,2)$ ). Допустим, что кривая $B$ либо не содержит неприводимых компонент с неотрицательным индексом самопересечения, либо имеет тип $1_{1,0,1}$ в $(V, C)$ и $B^{2}<3$. Пусть $B_{i}^{W}=\varphi_{\text {соб }}^{-1} B_{i}, B^{W}=\varphi^{-1}(B)=\varphi_{\text {соб }}^{-1} B+\sum_{i=1}^{l} E_{i}$, где $E_{i}-\varphi$-исключительные кривые на $W$ такие, что $\varphi\left(E_{i}\right) \in B$. Тогда из минимальности пары $(V, C)$, из теоремы 5.12 и леммы $5.9,3)$ несложно вывести, что ни одна из кривых $B_{i}^{W}$ не стягиваема в категории $X$. Поскольку морфизм $\varphi^{\prime}$ стягивает $\varphi_{\text {соб }}^{-1}$, первой неприводимой компонентой из $B^{W}$, стягиваемой морфизмом $\varphi^{\prime}$, является одна из кривых $E_{i}, i \in\{1, \ldots, l\}$. Теперь, используя лемму 4.4, получаем противоречие с тем, что $\left(\left(W, C^{W}\right), \varphi, \varphi^{\prime}\right) \in \mathrm{Ob}^{M} B_{2}(X)$. Лемма доказана.

УтВеРЖДЕНИЕ 7.8. Пусть $X$ - одна из категорий $R_{p_{\#}}$ при $4 / 5<p<1$ или $R^{N},\left(\left(W, C^{W}\right), \varphi, \varphi^{\prime}\right) \in \mathrm{Ob}^{M} B_{2}\left(X, X, \mathrm{Ob}^{M} X\right), \varphi:\left(W, C^{W}\right) \rightarrow(V, C), u F-$ неприводимая компонента кривой $C, k$ - число особых точек пары $(V, C)$, лежащих на $F, u(F, C-F)+k=2$. Тогда для любой неприводимой компонентьь $F_{i}^{W}$ кривой $F^{W}=\varphi^{-1}(F)$ имеем $\left(F_{i}^{W}, C^{W}-F_{i}^{W}\right)+k_{i}^{W}=2$, где $k_{i}^{W}$ - число особых точек пары $\left(W, C^{W}\right)$, лежащих на $F_{i}^{W}$.

ДокАЗАТЕЛьство. Предполагая противное, мы несложными рассуждениями придем к противоречию с тем, что $\left(\left(W, C^{W}\right), \varphi, \varphi^{\prime}\right) \in \mathrm{Ob}^{M} B_{2}\left(X, X, \mathrm{Ob}^{M} X\right)$. Утверждение доказано.

Лемма 7.9. Пусть $X$ - одна из категорий $R_{p_{\#}}$ при $4 / 5<p<1$ или $R^{N}$, $(V, C) \in \mathrm{Ob}^{M} X$ и кривая $F$ имеет mun $1_{2,0,2}$ в $(V, C)$, где $\left(F_{1}\right)^{2}=-r<0$, $\left(F_{2}\right)^{2}=0$, и является колесом. Тогда не существует такого $\left(\left(W, C^{W}\right), \varphi, \varphi^{\prime}\right) \in$ $\mathrm{Ob}^{M} B_{2}\left(X, X, \mathrm{Ob}^{M} X\right)$, что морфизм $\varphi^{\prime}$ стягивает кривую $\varphi_{\text {соб }}^{-1}\left(F_{1}\right)$.

ДокАЗАтельство. Допустим противное. Очевидно, можно считать, что $\varphi^{\prime}$ не стягивает компонент кривой $C$, не лежащих в $F$. Тогда согласно утверждению 7.3 имеем $\varphi(\operatorname{Ex}(\varphi)) \subset F$. Из утверждения 7.8 следует, что кривая $F^{W}=$ $\varphi^{-1}(F)$ имеет тип $1_{n^{W}, 0,2}$ в $\left(W, C^{W}\right)$. Пусть $\varphi=\sigma_{1} \circ \cdots \circ \sigma_{p}, \varphi^{\prime}=\sigma_{1}^{\prime} \circ \cdots \circ \sigma_{q}^{\prime}$, где $\sigma_{i}:\left(V^{i}, C^{i}\right) \rightarrow\left(V^{i-1}, C^{i-1}\right), \sigma_{i}^{\prime}:\left(\left(V^{\prime}\right)^{i},\left(C^{\prime}\right)^{i}\right) \rightarrow\left(\left(V^{\prime}\right)^{i-1},\left(C^{\prime}\right)^{i-1}\right), \sigma_{i}, \sigma_{i}^{\prime} \in$ $\operatorname{Mor}^{1} X,\left(V^{0}, C^{0}\right)=(V, C),\left(\left(V^{\prime}\right)^{0},\left(C^{\prime}\right)^{0}\right)=\left(V^{\prime}, C^{\prime}\right),\left(V^{p}, C^{p}\right)=\left(\left(V^{\prime}\right)^{q},\left(C^{\prime}\right)^{q}\right)=$ $\left(W, C^{W}\right), F^{i}=\left(\sigma_{1} \circ \cdots \circ \sigma_{i}\right)^{-1}(F), F_{1}^{i}=\left(\sigma_{1} \circ \cdots \circ \sigma_{i}\right)_{\text {соб }}^{-1}\left(F_{1}\right), \sigma_{i}-$ стягивание кривой $E^{i}$. Докажем, что $\sigma_{i}\left(E^{i}\right) \in F_{1}^{i-1}$. 
При $i=1$ утверждение очевидно.

Пусть $\sigma_{i}\left(E^{i}\right) \in F_{1}^{i-1}$ при $i \leqslant j$. Тогда кривая $F^{j}=\sum_{l=1}^{j+2} F_{l}^{j}$ имеет следующий вид: $\left(F_{1}^{j}\right)^{2}=-r-j,\left(F_{2}^{j}\right)^{2}=\left(F_{j+2}^{j}\right)^{2}=-1,\left(F_{l}^{j}\right)^{2}=-2$ при $2<l<j+2$. Допустим, что $\sigma_{j+1}\left(E^{j+1}\right) \notin F_{1}^{j}$, тогда $\sigma_{j+1}\left(E^{j+1}\right)=F_{s}^{j} \cap F_{s+1}^{j}$, где $s \neq 1, j+2$. Легко видеть, что если кривая $\left(\sigma_{l}^{\prime} \circ \cdots \circ \sigma_{q}^{\prime}\right)_{*} \circ\left(\sigma_{j+2} \circ \cdots \circ \sigma_{p}\right)^{-1}\left(E^{j+1}\right)$ не стягивается морфизмом $\sigma_{l-1}^{\prime}$, то кривые $\left(\sigma_{l}^{\prime} \circ \cdots \circ \sigma_{q}^{\prime}\right)_{*} \circ\left(\sigma_{j+2} \circ \cdots \circ \sigma_{p}\right)_{\text {соб }}^{-1}\left(F_{s}^{j}\right)$ и $\left(\sigma_{l}^{\prime} \circ \cdots \circ \sigma_{q}^{\prime}\right)_{*} \circ\left(\sigma_{j+2} \circ \cdots \circ \sigma_{p}\right)_{\text {соб }}^{-1}\left(F_{s+1}^{j}\right)$ также не стягиваются морфизмом $\sigma_{l-1}^{\prime}$. Поэтому из условия $\left(V^{\prime}, C^{\prime}\right) \in \mathrm{Ob}^{M} X$ следует, что

$$
\left(\left(W, C^{W}\right), \varphi, \varphi^{\prime}\right) \underset{B_{2}(X)}{>}\left(\left(W^{\prime}, C^{W^{\prime}}\right), \psi, \psi^{\prime}\right),
$$

где $\sigma:\left(W, C^{W}\right) \rightarrow\left(W^{\prime}, C^{W^{\prime}}\right)$ - стягивание кривой $\left(\sigma_{j+2} \circ \cdots \circ \sigma_{p}\right)^{-1}\left(E^{j+1}\right)$. Получаем противоречие с тем, что $\left(\left(W, C^{W}\right), \varphi, \varphi^{\prime}\right) \in \mathrm{Ob}^{M} B_{2}\left(X, X, \mathrm{Ob}^{M} X\right)$.

Таким образом, $\sigma_{i}\left(E^{i}\right) \in F_{1}^{i-1}$ и кривая $F^{W}=F^{p}=\sum_{l=1}^{p+2} F_{l}^{W}$ имеет следующий вид: $\left(F_{1}^{W}\right)^{2}=-r-p,\left(F_{2}^{W}\right)^{2}=\left(F_{p+2}^{W}\right)^{2}=-1,\left(F_{l}^{W}\right)^{2}=-2$ при $2<l<p+2$. Тогда очевидно, что кривая $F_{1}^{W}$ не стягивается морфизмом $\varphi^{\prime}$ и $\left(\varphi_{*}^{\prime} F_{1}^{W}\right)^{2}=-r$. Лемма доказана.

ЗАмечание 7.10 . Пусть $(V, C) \in \mathrm{Ob} R, B_{i}$ - неприводимые кривые типа $0_{n^{i}, m^{i}, k^{i}}$ в $(V, C)$, где $k^{i} \leqslant 2$, и $\left(B_{i}\right)^{2}=\left(B_{i}, B_{k}\right)=0$. Тогда:

1) согласно теореме $3.4,5)$ кривые $B_{i}$ численно пропорциональны, в случае, когда $B_{i}$ не содержат особенностей пары $(V, C)$, из формулы присоединения следует их численная эквивалентность;

2) из леммы $5.9,1)$ и леммы 6.1 следует, что кривая $B_{i}$ либо не содержит особенностей пары $(V, C)$, либо содержит две особые точки типов $0_{n_{1}^{i}, 1, k_{1}^{i}}$ и $0_{n_{2}^{i}, 1, k_{2}^{i}}$ в $(V, C)$. В последнем случае $(V, C) \notin \mathrm{Ob} \widetilde{R}_{N}^{M}$.

УтвеРЖДЕНИЕ 7.11. Пусть $(V, C) \in \mathrm{Ob} R^{N}, F=\sum_{i=1}^{n} F_{i}-$ кривая типа $0_{n, m, k}$ или $1_{n, 0, k}$ в $(V, C)$, где $k \leqslant 2$ (в последнем случае $F$ - колесо), $p$ число неприводимых компонент $F_{i}$ кривой $F$ с неотрицателъным индексом самопересечения. Тогда:

1) $p \leqslant 4$, и если $p=4$, mо $n=4, F$ uмеет mun $1_{4,0,2} u\left(F_{i}\right)^{2}=0$ nрu $i \in\{1, \ldots, 4\}$;

2) если $F$ содержит две непересекающиеся компоненты $F_{i}, F_{j} c\left(F_{i}\right)^{2} \geqslant 0$, $\left(F_{j}\right)^{2} \geqslant 0$, mо $\left(F_{i}\right)^{2}=\left(F_{j}\right)^{2}=0$, и если $F$ имеет mun $0_{n, m, k}$, mo $n=3$, а если $F$ uмeеm mun $1_{n, 0, k}$, mo $n=4$;

3) если $F$ содержит две компоненты $F_{i}, F_{j} c\left(F_{i}\right)^{2}>0,\left(F_{j}\right)^{2}>0$, то если $F$ имеет тип $0_{n, m, k}$, то $n=2,\left(F_{i}\right)^{2}=\left(F_{j}\right)^{2}=1$, а если $F$ имеет тuп $1_{n, 0, k}$, то $n \leqslant 3 u\left(F_{i}\right)^{2}=\left(F_{j}\right)^{2}=1$ nрu $n=3 u 1 \leqslant\left(F_{i}\right)^{2} \leqslant 2,1 \leqslant\left(F_{j}\right)^{2} \leqslant 2$ npu $n=2$.

ДокАЗАтельство. Допустим, что $p \geqslant 4$; тогда существуют неприводимые компоненты $F_{i_{1}}, F_{i_{2}}, F_{i_{3}}, F_{i_{4}}$ кривой $F,\left(F_{i_{j}}\right)^{2} \geqslant 0$ при $j \in\{1, \ldots, 4\}$, такие, что $F_{i_{j}} \neq F_{i_{l}}$, если $j \neq l$. Из теоремы $\left.3.4,1\right)$ следует, что $\left(F_{i_{j}}\right)^{2}=0$ при $j \in\{1, \ldots, 4\}$, поскольку в противном случае одна из компонент пересекала бы три оставшиеся. Очевидно, что среди $F_{i_{j}}$ не может быть трех попарно непересекающихся компонент, так как если бы, например, $F_{i_{1}}, F_{i_{2}}$ и $F_{i_{3}}$ не пересекались, то из замечания $7.10,1)$ следовало бы, что они численно эквивалентны, что невозможно. Таким образом, единственным возможным вариантом (по 
модулю нумерации) является следующий:

$$
\left(F_{i_{1}}, F_{i_{2}}\right)=\left(F_{i_{2}}, F_{i_{3}}\right)=\left(F_{i_{3}}, F_{i_{4}}\right)=\left(F_{i_{4}}, F_{i_{1}}\right)=1 .
$$

Поэтому $F$ имеет тип $1_{4,0,2}$ и утверждение 1 ) доказано.

Из теоремы $3.4,1)$ следует, что $\left(F_{i}\right)^{2}=\left(F_{j}\right)^{2}=0$. Теперь утверждение 2 ) очевидным образом вытекает из того, что $F_{i}$ и $F_{j}$ численно эквивалентны согласно замечанию $7.10,1)$.

Осталось доказать утверждение 3). Согласно теореме $3.4,1$ ) выполнено $\left(F_{i}, F_{j}\right)>0$. Следовательно, $\left(F_{i}, F_{j}\right)=1,2$. Предположим, что $F$ имеет либо тип $1_{n, 0, k}$ и $n \geqslant 3$, либо тип $0_{n, m . k}$. В этом случае $\left(F_{i}, F_{j}\right)=1$. Пусть $\sigma:\left(V^{\prime}, C^{\prime}\right) \rightarrow(V, C)$ - раздутие точки пересечения $F_{i}$ и $F_{j}$; тогда $\left(\sigma_{\text {соб }}^{-1}\left(F_{i}\right)\right)^{2} \geqslant 0$, $\left(\sigma_{\text {соб }}^{-1}\left(F_{j}\right)\right)^{2} \geqslant 0,\left(\sigma_{\text {соб }}^{-1}\left(F_{i}\right), \sigma_{\text {соб }}^{-1}\left(F_{j}\right)\right)=0$. Из утверждения 2$)$ следует, что $\left(\sigma_{\text {соб }}^{-1}\left(F_{i}\right)\right)^{2}=0,\left(\sigma_{\text {соб }}^{-1}\left(F_{j}\right)\right)^{2}=0$. Поэтому $\left(F_{i}\right)^{2}=\left(F_{j}\right)^{2}=1$ и из численной эквивалентности $\sigma_{\text {соб }}^{-1}\left(F_{i}\right)$ и $\sigma_{\text {соб }}^{-1}\left(F_{j}\right)$ вытекает, что если $F$ имеет тип $0_{n, m, k}$, то $n=2$, а если $F$ имеет тип $1_{n, 0, k}$, то $n=3$. В случае, когда $F$ имеет тип $1_{n, 0, k}$ и $n=2$, мы аналогично получаем, что $\left(F_{i}\right)^{2} \leqslant 2,\left(F_{j}\right)^{2} \leqslant 2$. Утверждение доказано.

СЛЕДСТВИЕ 7.12. Пусть $X$ - одна из категорий $R^{N}, R_{p_{\#}}$ nрu $4 / 5<p<1$ или $R_{1_{-}},(V, C) \in \mathrm{Ob} \widetilde{X}_{N}^{M}$. Тогда если $F=\sum_{i=1}^{n} F_{i}$ - кривая типа $0_{n, m, k}$ в $(V, C)$, где $k \leqslant 2$, содержащая неприводимую компоненту с неотрицательным индексом самопересечения, то из утверждения 7.11 следует, что кривая $F$ имеет один из следующих численных типов в $(V, C)$ :

1) $0_{n, m, k}\left[(a)_{i}\right]$, где $a \geqslant 0$;

2) $0_{n, m, k}\left[(a)_{i},(b)_{i+1}\right]$, где $a, b \geqslant 0$ и либо $a=0$, либо $b=0$;

3) $0_{3, m, 2}\left[(0)_{1},(0)_{3}\right]$, в этом случае из замечания $\left.7.10,1\right)$ следует, что $m=0$ или $m=2$;

4) $0_{2, m, k}\left[(1)_{1},(1)_{2}\right]$, из замечания $\left.7.10,1\right)$ следует, что $m=0$ или $m=2$.

ОПРЕДЕЛЕНИЕ 7.13. Пусть $X$ - одна из категорий $R^{N}, R_{p_{\#}}$ при $4 / 5<p<1$ или $R_{1_{-}},(V, C) \in \mathrm{Ob} \widetilde{X}_{N}^{M}$. Тогда пару $(V, C)$ мы будем называть стандартной, если кривая $F_{X}(V, C)$ либо состоит из более чем одной связной компоненты, либо имеет численный тип $0_{n, m, k}\left[a_{i}\right]$ в $(V, C)$.

ОПРЕДЕЛЕНИЕ 7.14. Пусть $(V, C) \in \mathrm{Ob} R^{N}, F=\sum_{j=1}^{n} F_{j}-$ кривая типа $0_{n, m, k}$ или $F$ - колесо типа $1_{n, 0, k}$ в $(V, C)$, где $k \leqslant 2$, и пусть кривая $F$ содержит неприводимую компоненту $F_{i}$ с неотрицательным индексом самопересечения, $\left(F_{i}\right)^{2}=a$ и любая неприводимая компонента $F_{j}$ кривой $F$ неособа. Пусть $p \in F$ - точка на кривой $F_{i}$ такая, что если $\left(F_{i}, C-F_{i}\right)=2$, то $p \in F_{i} \cap\left(C-F_{i}\right)$. Введем морфизмы $\varepsilon_{F_{i}, p} \in \operatorname{Mor} \widetilde{R}^{N}$ как $\varepsilon_{F_{i}, p}=\sigma^{\prime} \circ\left(\sigma_{p}\right)^{-1}$, где $\sigma_{p}:\left(V^{\prime}, C^{\prime}\right) \rightarrow(V, C)$ - последовательность стягиваний, $\sigma_{p}=\sigma_{1} \circ \cdots \circ \sigma_{a+1}$, такая, что $\sigma_{j}:\left(V^{j}, C^{j}\right) \rightarrow\left(V^{j-1}, C^{j-1}\right), \sigma_{j} \in \operatorname{Mor}^{1} R^{N},\left(V^{0}, C^{0}\right)=(V, C)$, $\left(V^{a+1}, C^{a+1}\right)=\left(V^{\prime}, C^{\prime}\right), E_{j}-\sigma_{j}$-исключительные кривые, $\sigma_{j}\left(E_{j}\right)=\left(\sigma_{1} \circ \cdots\right.$ $\left.\cdots \circ \sigma_{j-1}\right)_{\text {соб }}^{-1} F_{i} \cap E_{j-1}$ при $j>2, \sigma_{1}\left(E_{1}\right)=p$ и и $\sigma^{\prime}:\left(V^{\prime}, C^{\prime}\right) \rightarrow\left(V^{\prime \prime}, C^{\prime \prime}\right)-$ стягивание кривой $G^{\prime} \cup H^{\prime}$. Заметим, что $\left(\left(\sigma_{p}\right)_{\text {соб }}^{-1} F_{i}\right)^{2}=-1$.

Пусть $E_{j}^{\prime}$ - собственные прообразы определенных выше кривых $E_{j}$ на $V^{\prime}$; тогда $\left(E_{a+1}^{\prime}\right)^{2}=-1,\left(E_{j}^{\prime}\right)^{2}=-2$ при $j \leqslant a$. Определим кривые $G^{\prime}$ и $H^{\prime}$ на $V^{\prime}$ следующим образом: $G^{\prime}:=\sum_{j=1}^{n_{G}} G_{j}-$ кривая численного типа

$$
0_{n_{G}, m_{G}, k_{G}}\left[(-1)_{1},(-2)_{2},(-2)_{3}, \ldots,(-2)_{n_{G}}\right]
$$


в $(V, C)$, где $k_{G} \leqslant 2, G^{\prime} \subset F^{\prime}$, такая, что $G_{1}^{\prime}=\left(\sigma_{p}\right)_{\text {соб }}^{-1}\left(F_{i}\right)$, и $n_{G} \in \mathbb{Z}_{\geqslant 0}-$ максимальное число с такими свойствами,

$$
H^{\prime}:= \begin{cases}\left(\sigma_{p}\right)_{\text {соб }}^{-1}(D)+\sum_{j=1}^{a} E_{j}^{\prime}, & \text { если } p=D \cap F_{i}, \text { где } D- \\ & \text { неприводимая компонента кривой } F \\ & \text { с } D^{2}=0 \text { и }\left(\left(\sigma_{p}\right)_{\text {соб }}^{-1}(D), G^{\prime}\right)=0, \\ & \text { в противном случае. }\end{cases}
$$

Очевидно, что такое определение корректно и $\sigma^{\prime} \in \operatorname{Mor} R^{N}$. Заметим, что если $(V, C) \in \mathrm{Ob}^{M} R^{N}$, то $\left(V^{\prime \prime}, C^{\prime \prime}\right) \in \mathrm{Ob}^{M} R^{N}$ и $\varepsilon_{F_{i}, p} \in \operatorname{Mor} \widetilde{R}^{N M}$.

ОПРедЕЛЕНиЕ 7.15. Пусть $(V, C) \in \mathrm{Ob} R^{N}, G=\sum_{i=1}^{n} G_{i}$ - минималиная кривая численного типа $0_{n, m, k}\left[\left(a^{1}\right)_{1}\right]$ в $(V, C)$ такая, что $a^{i}=\left(G_{i}\right)^{2}$ и $a^{1} \geqslant 0$. Численной степенъю $d(G)$ кривой $G$ называется число

$$
d(G):=\sum_{i=2}^{n}-a^{i}
$$

Очевидно, что $d(G) \geqslant 0$ и $d(G)=0$ тогда и только тогда, когда $G=G_{1}$.

ЗАмЕчАниЕ 7.16. В условиях определения 7.15 пусть $\varepsilon_{G_{1}, p}:(V, C)-\rightarrow$ $\left(V^{\prime \prime}, C^{\prime \prime}\right)$ - морфизм категории $\widetilde{R}^{N}$ (см. определение 7.14), где $p \notin \operatorname{Sing} G$. Определим кривую $G^{\prime \prime}$ в $\left(V^{\prime \prime}, C^{\prime \prime}\right)$ следующим образом:

$$
G^{\prime \prime}:=\sum_{i=1}^{q} G_{i}^{\prime \prime}=\sigma_{*}^{\prime}\left(E_{a^{1}+1}^{\prime}\right)+\sigma_{*}^{\prime} \circ\left(\sigma_{p}\right)_{\operatorname{co\sigma }}^{-1}\left(\sum_{i=2}^{n} G_{i}\right),
$$

где $G_{1}^{\prime \prime}=\sigma^{\prime}\left(E_{n_{1}+1}^{\prime}\right)$. Тогда $G^{\prime \prime}-$ кривая численного типа $0_{q, m, l}\left[\left(b^{1}\right)_{1}\right]$ в $(V, C)$, где $l \leqslant 2$ и $b^{1} \geqslant 0$, и из определения 7.14 следует,что $d\left(G^{\prime \prime}\right)<d(G)$.

ОПредЕлЕНИЕ 7.17. Пусть $X$ - одна из категорий $R_{p_{\#}}$ при $p>4 / 5$ или $R^{N}$, $(V, C) \in \mathrm{Ob}^{M} X$ и $F-$ кривая на $V$. Тогда мы будем говорить, что $F$ yдовлетворяет требованию $(*)$ в категории $X$, если любая максимальная связная компонента $B$ кривой $F$ содержит неприводимую компоненту с неотрицательным индексом самопересечения и $F$ удовлетворяет одному из следующих условий:

1) $F$ имеет тип $0_{n, m, k}$ в $(V, C)$, где $m, k \leqslant 2$ и $m=0$ в случае $X=R_{1}$;

2) $F$ состоит из нескольких неприводимых непересекающихся компонент с нулевым индексом самопересечения, имеющих тип $0_{n_{i}, 0, k_{i}}$ или $0_{1, m, m}$ в $(V, C)$, где $k_{i}, m \leqslant 2$, и $m=0$ в случае $X=R_{1}$;

3) $X \neq R_{1}, F$ - колесо, и если $F$ имеет тип $1_{1,0,1}$ в $(V, C)$, то $F^{2} \geqslant 3$, а если $F$ имеет тип $1_{2,0,2}$, то по модулю нумерации $F \neq F_{1}+F_{2}$, где $\left(F_{1}\right)^{2}<0,\left(F_{2}\right)^{2}=0$.

ЗАмечАниЕ 7.18. Пусть $X$ - одна из категорий $R_{p_{\#}}$ при $p>4 / 5$ или $R^{N}$, $(V, C) \in \mathrm{Ob}^{M} X$ и $F_{1}, F_{2}$ - кривые на $V$, удовлетворяющие требованию $(*)$. Тогда из теоремы $3.4,1), 4)$ и замечания 7.10 следует, что кривая $F_{1} \cup F_{2}$ удовлетворяет требованию $(*)$.

ТеОрема 7.19. Пусть $X$ - одна из категорий $R_{p_{\#}}$ при $p>4 / 5$ или $R^{N}$, $(V, C) \in \mathrm{Ob}^{M} X$ и $F=F_{X}(V, C)$. Тогда $F$ удовлетворяет требованию $(*)$. 
ДокАЗАТЕльство. Согласно лемме 7.7 любая максимальная связная компонента $B$ кривой $F$ содержит неприводимую компоненту с неотрицательным индексом самопересечения. Из леммы 7.6 следует, что $B$ имеет тип $0_{n, m, k}$ в $(V, C)$, где $m, k \leqslant 2$ (и $m=0$, компонента $B$ неприводима и $(B, C-B)=0$ в случае $X=R_{1}$ ), или является колесом при $X \neq R_{1}$. Из замечания 7.10 и теоремы 3.4 вытекает, что если $F$ состоит из более чем одной максимальной связной компоненты, то каждая из них неприводима, имеет нулевой индекс самопересечения и тип $0_{n_{i}, 0, k_{i}}$ или $0_{1, m, m}$ в $(V, C)$ и $m=0$ при $X=R_{1}$. Из лемм $7.7,7.9$ следует, что $F$ не может иметь тип $1_{1,0,1}$ в $(V, C)$ с $F^{2} \geqslant 3$, а также тип $1_{2,0,2}$ и состоять из двух неприводимых компонент $F_{1}$ и $F_{2}$, где $\left(F_{1}\right)^{2}<0,\left(F_{2}\right)^{2}=0$.

Tеорема 7.20. Пусть $(V, C) \in \mathrm{Ob}^{M} R^{N}, F=\sum_{i=1}^{n} F_{i}$-максимальная кривая в $(V, C)$, удовлетворяющая требованию $(*)$ (m. е. не существует кривой $G$, удовлетворяющей условию (*) и такой, что $F \subset G, F \neq G)$. Тогда:

1) $F=F_{R^{N}}(V, C)$;

2) если $F$ - кривая типа $0_{n, m, k}$ в $(V, C)$, отличного от $0_{3, m, 2}\left[0_{1}, 0_{3}\right]$ u $0_{2, m, k}\left[1_{1}, 1_{2}\right]$, то существует $\gamma \in \operatorname{Mor} \widetilde{R}^{N M}$ maкое, что пара $\left(V^{\prime}, C^{\prime}\right)$ стандартна, где $\left(\left(W, C^{W}\right), \varphi, \varphi^{\prime}\right) \in L_{R^{N}}(\gamma), \varphi:\left(W, C^{W}\right) \rightarrow(V, C), \varphi^{\prime}:\left(W, C^{W}\right) \rightarrow$ $\left(V^{\prime}, C^{\prime}\right)$.

ДокАзАтельство. То, что кривая $F_{R^{N}}(V, C)$ удовлетворяет требованию $(*)$, доказано в теореме 7.19. Из замечания 7.18 следует, что для доказательства теоремы необходимо доказать следующее

УтвеРЖДЕНИЕ 7.21. Пусть $F=\sum_{i=1}^{n} F_{i}-$ кривая типа $0_{n, m, k}$ или $1_{n, 0, k}$ в $(V, C)$, где $k \leqslant 2$, удовлетворяющая требованию $(*)$. Тогда:

а) для любой компоненты $F_{i}$ кривой $F$ существует $\gamma \in$ Mor $\widetilde{R}^{N M}$ такое, что $\varphi^{\prime} \circ \varphi_{\text {соб }}^{-1}\left(F_{i}\right)=\mathrm{pt}$, где $\left(\left(W, C^{W}\right), \varphi, \varphi^{\prime}\right) \in L_{R^{N}}(\gamma), \varphi:\left(W, C^{W}\right) \rightarrow(V, C)$;

b) если кривая $F$ имеет численный тuп $0_{n, m, k}\left[(a)_{i},(b)_{i+1}\right]$, где $a, b \geqslant 0, u$ не существует $\rho \in \operatorname{Mor} \widetilde{R}^{N M}$ такого, что кривая $\psi_{*}^{\prime \prime} \psi^{-1}(F)$ имеет численный mun $0_{2, m, k}\left[(1)_{1},(1)_{2}\right]$ в $\left(V^{\prime \prime}, C^{\prime \prime}\right)$, где $\left(\left(Z, C^{Z}\right), \psi, \psi^{\prime \prime}\right) \in L_{R^{N}}(\rho), \psi:\left(Z, C^{Z}\right) \rightarrow$ $(V, C), \psi^{\prime \prime}:\left(Z, C^{z}\right) \rightarrow\left(V^{\prime \prime}, C^{\prime \prime}\right)$, то либо $a=0$, либо $b=0$ и существует морфизм $\gamma \in \operatorname{Mor} \widetilde{R}^{N M}$ такой, что кривая $F^{\prime}=\varphi_{*}^{\prime} \circ \varphi^{-1}(F)$ имеет численный muп $0_{n^{\prime}, m, k^{\prime}}\left[(c)_{1}\right]$ в $\left(V^{\prime}, C^{\prime}\right)$, где $c \geqslant 0,\left(\left(W, C^{W}\right), \varphi, \varphi^{\prime}\right) \in L_{R^{N}}(\gamma)$, $\varphi:\left(W, C^{W}\right) \rightarrow(V, C), \varphi^{\prime}:\left(W, C^{W}\right) \rightarrow\left(V^{\prime}, C^{\prime}\right)$.

ДокАЗАТЕЛЬСтво. В случае, если $F$ имеет тип $1_{1,0,1}$, произведем раздутие $\sigma:\left(V^{\prime}, C^{\prime}\right) \rightarrow(V, C)$ единственной особой точки кривой $F$. Пусть $F^{\prime}=\sigma_{\text {соб }}^{-1}(F)$; тогда $\left(F^{\prime}\right)^{2}=\left(\sigma_{\text {соб }}^{-1} F\right)^{2}=F^{2}-4$. Если $\left(F^{\prime}\right)^{2}=-1$, стянем кривую $F^{\prime}$, а если $\left(F^{\prime}\right)^{2} \geqslant 0$, применим к паре $\left(V^{\prime}, C^{\prime}\right)$ морфизм $\varepsilon_{F^{\prime}, p^{\prime}} \in \operatorname{Mor} \widetilde{R}^{N M}$, где $p^{\prime} \in F^{\prime} \cap \operatorname{Sing} C^{\prime}$. Пусть $\left(\left(Z, C^{Z}\right), \pi^{\prime}, \pi^{\prime \prime}\right) \in L_{R^{N}}\left(\varepsilon_{F^{\prime}, p^{\prime}}\right)$. Очевидно, что морфизм $\pi^{\prime \prime}$ стягивает кривую $\left(\pi^{\prime}\right)_{\text {соб }}^{-1}\left(F^{\prime}\right)$. Это доказывает утверждение для данного случая.

Предположим теперь, что если $F$ имеет тип $1_{n, 0, k}$, то $n \geqslant 2$. Тогда если $F_{i}$ - компонента кривой $F$ с $\left(F_{i}\right)^{2} \geqslant 0$, то морфизм $\varepsilon_{F_{i}, p} \in \operatorname{Mor} R^{N M}$ удовлетворяет требованиям утверждения а). Таким образом, утверждение а) в случае $\left(F_{i}\right)^{2} \geqslant 0$ доказано.

Доказательства утверждения а) в случае $\left(F_{i}\right)^{2}<0$ и утверждения b) аналогичны. Для доказательства а) в случае, когда $F$ имеет тип $1_{2,0,2}$, раздуем любую особую точку кривой $F, \sigma:\left(V^{\prime}, C^{\prime}\right) \rightarrow(V, C)$, получившуюся при этом 
пару $\left(V^{\prime}, C^{\prime}\right)$ и кривые $F^{\prime}=\sigma^{-1}(F), F_{i}^{\prime}=\sigma_{\text {соб }}^{-1}\left(F_{i}\right)$ во избежание громоздкости записи мы будем обозначать далее через $(V, C), F$ и $F_{i}$ соответственно.

Для доказательства утверждений а), b) построим кривую $G=\sum_{i=1}^{n_{G}} G_{i}$ типа $0_{n_{G}, m_{G}, k_{G}}$ в $(V, C)$ такую, что $\left(G_{1}\right)^{2} \geqslant 0,\left(G_{l}\right)^{2} \leqslant-2$ при $l>1$. В случае доказательства утверждения а) заметим, что существует компонента $F_{j}$ кривой $F$ с $\left(F_{j}\right)^{2} \geqslant 0$. Положим $G_{1}=F_{j}, G_{n_{G}}=F_{i}$. В доказательстве утверждения b) положим $G_{1}=F_{i}, G_{n_{G}}=F_{i}$.

Если $d(G)>0$, применим к паре $(V, C)=\left(V^{0}, C^{0}\right)$ морфизм $\varepsilon_{1}=\varepsilon_{G_{1}^{0}, p^{0}} \in$ Mor $\widetilde{R}^{N M}, \varepsilon_{1}:\left(V^{0}, C^{0}\right) \rightarrow\left(V^{1}, C^{1}\right)$, где $p^{0} \notin \operatorname{Sing} G^{0}, G^{0}=G, G_{l}^{0}=G_{l}$. Определим кривую $G^{1}$ на $V^{1}$ по аналогии с определением $G^{\prime \prime}$ в замечании 7.16. Согласно этому же замечанию имеем $d\left(G^{1}\right)<d\left(G^{0}\right)$. Если $d\left(G^{1}\right)=0$, положим $\varepsilon=\varepsilon_{1}$, а если $d\left(G^{1}\right)>0$, аналогичным образом строим морфизм $\varepsilon_{2}=\varepsilon_{G_{1}^{1}, p^{1}} \in$ Mor $\widetilde{R}^{N M}, \varepsilon_{2}:\left(V^{1}, C^{1}\right) \rightarrow\left(V^{2}, C^{2}\right)$, и так далее, пока $d\left(G^{s}\right) \neq 0$. В результате получаем морфизм $\varepsilon \in \operatorname{Mor} \widetilde{R}^{N M}, \varepsilon=\varepsilon_{s} \circ \cdots \circ \varepsilon_{1}, \varepsilon:\left(V^{0}, C^{0}\right) \rightarrow\left(V^{s}, C^{s}\right)$, такой, что $d\left(G^{s}\right)=0$. Пусть $\left(\left(W, C^{W}\right), \varphi, \varphi^{\prime}\right) \in L_{R^{N}}(\varepsilon)$. В случае доказательства утверждения а) морфизм $\varphi^{\prime}$ стягивает кривую $\varphi_{\text {соб }}^{-1}\left(F_{i}\right)$, и утверждение а) доказано.

В случае доказательства утверждения b) легко видеть, что кривая $F^{s}=$ $\varphi_{*}^{\prime} \varphi^{-1}(F)$ имеет численный тип $0_{n^{s}, m, k^{s}}\left[\left(a^{s}\right)_{1}\right]$ или $0_{n^{s}, m, k^{s}}\left[a_{1}^{s}, b_{2}^{s}\right]$ в $\left(V^{s}, C^{s}\right)$, где $a^{s}, b^{s} \geqslant 0$ и $a^{s}=0$ или $b^{s}=0$. Если $a^{s} \neq 0$, применим к паре $\left(V^{s}, C^{s}\right)$ морфизм $\varepsilon_{F_{2}^{s}, p^{s}} \in \operatorname{Mor} \widetilde{R}^{N M}, \varepsilon_{F_{2}^{s}, p^{s}}:\left(V^{s}, C^{s}\right) \rightarrow\left(V^{s+1}, C^{s+1}\right)$, где $p^{s}=F_{1}^{s} \cap F_{2}^{s}$. Тогда $F^{s+1}=\varepsilon_{F_{2}^{s}, p^{s}}\left(F^{s}\right)$ имеет численный тип $0_{n^{s+1}, m, k^{s+1}}\left[\left(a^{s}-1\right)_{1},(0)_{2}\right]$. Продолжая этот процесс, мы придем к паре $\left(V^{t}, C^{t}\right)$ такой, что кривая $F^{t}$ имеет численный тип $0_{n^{t}, m, k^{t}}\left[(0)_{1},\left(b^{t}\right)_{2}\right]$ в $\left(V^{t}, C^{t}\right)$. Если $b^{t} \geqslant 0$, то, применив к паpe $\left(V^{t}, C^{t}\right)$ последовательность морфизмов $\varepsilon_{b^{t}+t} \circ \cdots \circ \varepsilon_{t}$ категории $\widetilde{R}^{N M}$, где $\varepsilon_{j}=\varepsilon_{F_{1}^{j}, p^{j}}, p^{j}=F_{1}^{j} \cap F_{2}^{j}$ при $j \in\left\{t, \ldots, b^{t}+t\right\}$, мы получим пару $\left(V^{z}, C^{z}\right)$ такую, что кривая $F^{z}$ имеет численный тип $0_{n^{z}, m, k^{z}}\left[\left(a^{z}\right)_{1}\right]$, где $a^{z} \geqslant 0, z=b^{t}+t+1$, что доказывает утверждение b). Таким образом, доказано утверждение, а следовательно, и теорема.

Tеорема 7.22. Пусть $X$ - одна из категорий $R_{p_{\#}}$ nри $4 / 5<p<1$ или $R_{1_{-}}$. Тогда:

1) если $(V, C) \in \operatorname{Ob} X u\left(\left(W, C^{W}\right), \varphi\right) \in N_{X}(V, C), \operatorname{mo} F_{R^{N}}\left(W, C^{W}\right)=$ $\varphi^{-1}\left(F_{X}(V, C)\right)$

2) если $(V, C) \in \mathrm{Ob}^{M} X$ и $F=\sum_{i=1}^{n} F_{i}-$ максимальная кривая в $(V, C)$, удовлетворяющая условию $(*)$, то $F=F_{X}(V, C)$;

3) если $(V, C) \in \operatorname{Ob} \widetilde{X}_{N}^{M}$ и F имеет тuп $0_{n, m, k}$ в $(V, C)$, отличный от $0_{3, m, 2}\left[0_{1}, 0_{3}\right]$ u $0_{2, m, k}\left[1_{1}, 1_{2}\right]$, то существует морфизм $\gamma \in \operatorname{Mor} \widetilde{X}_{N}^{M}, \gamma:(V, C)--\rightarrow$ $\left(V^{\prime}, C^{\prime}\right)$, такой, что пара $\left(V^{\prime}, C^{\prime}\right)$ стандартна.

ДоказАтельство. То, что кривая $F_{X}(V, C)$ удовлетворяет требованию $(*)$, доказано в теореме 7.19.

Согласно замечанию $7.4,1)$ можно считать, что $(V, C) \in \mathrm{Ob} \widetilde{X}_{N}^{M}$. Очевидно, что $\varphi_{*}\left(F_{R^{N}}\left(W, C^{W}\right)\right) \subset F_{X}(V, C)$. Из того, что кривая $F_{X}(V, C)$ удовлетворяет требованию $(*)$, из замечания 7.10 , того факта, что $\varphi(\operatorname{Ex}(\varphi)) \cap F_{X}(V, C)=\varnothing$, если кривая $F_{X}(V, C)$ имеет тип $1_{n, 0, k}$, и теоремы 7.20 следует, что кривая $\varphi^{-1} F_{X}(V, C)$ удовлетворяет требованию $(*)$. Поэтому $\varphi^{-1}\left(F_{X}(V, C)\right) \subset$ $F_{R^{N}}\left(W, C^{W}\right)$ и $\varphi^{-1}\left(F_{X}(V, C)\right)=F_{R^{N}}\left(W, C^{W}\right)$, что доказывает утверждение 1$)$. 
Отсюда согласно утверждению 1) теоремы 7.20 и лемме 6.1 следует истинность утверждения 2). Утверждение 3) следует из теоремы 7.20,2). Теорема доказана.

ЗАмЕЧАНИЕ 7.23. 1) Из утверждения 1) теоремы 7.22 и следствия $5.13,2$ ) вытекает, что если $(V, C) \in \mathrm{Ob} R_{p_{1}}$ при $4 / 5<p_{1}<1$, то $(V, C) \in \mathrm{Ob} R_{p_{2}} \subset$ $\mathrm{Ob} R_{1_{-}}$при любом $p_{2}, p_{1}<p_{2}<1$, и $F_{R_{p_{1}}}(V, C)=F_{R_{p_{2}}}(V, C)=F_{R_{1_{-}}}(V, C)$.

2) Пусть $(V, C) \in \mathrm{Ob} \widetilde{X}_{N}^{M}$, где $X$ - одна из категорий $R_{p_{\#}}$ при $4 / 5<p<1$ или $R_{1_{-}}, F=F_{X}(V, C)$ и $F_{X}^{\prime}(V, C)$ - кривая на $V$, определяемая следующим образом:

$$
F_{X}^{\prime}(V, C):=\sum_{i \in I} E_{i}
$$

где $E_{i}$ - неприводимые кривые на $V$ и $i \in I$, если существует объект $\left(\left(W_{i}, C^{W_{i}}\right)\right.$, $\left.\varphi_{i}, \varphi_{i}^{\prime}\right)$ категории $B_{2}\left(X, X, \mathrm{Ob} \widetilde{X}_{N}^{M}\right)$, где морфизм $\varphi_{i}:\left(W_{i}, C^{W_{i}}\right) \rightarrow(V, C)$ такой, что $\varphi_{i}^{\prime}$ стягивает кривую $\left(\varphi_{i}\right)_{\text {соб }}^{-1}\left(E_{i}\right)$. Тогда из того, что определенные выше элементарные морфизмы $\varepsilon_{F_{i}, p}:(V, C)-\rightarrow\left(V^{\prime}, C^{\prime}\right)$ принадлежали множеству Mor $\widetilde{R}^{N M}$, и из замечания $\left.7.4,1\right)$ следует $F_{X}(V, C)=F_{X}^{\prime}(V, C)$.

3) При $p<4 / 5$ утверждение 1) теоремы 7.22 может не выполняться.

Пример 7.24. Пусть $p=1 / 2, V$ - неособая поверхность; $C=\sum_{i=1}^{3} C_{i}+$ $\sum_{i=1}^{3} C_{i}^{\prime}$ - кривая на $V$ такая, что $C_{i}, C_{i}^{\prime}$ неособы, рациональны и $\left(C_{i}\right)^{2}=$ $\left(C_{i}^{\prime}\right)^{2}=-1,\left(C_{i}, C_{j}\right)=\left(C_{i}^{\prime}, C_{j}^{\prime}\right)=0$ при $i \neq j,\left(C_{i}, C_{j}^{\prime}\right)=1$ при любых $i, j$. Пусть $\varphi_{1}:(V, C) \rightarrow\left(V^{1}, C^{1}\right)$ - стягивание всех кривых $C_{i}$, а $\varphi_{2}:(V, C) \rightarrow$ $\left(V^{1}, C^{1}\right)$ - стягивание всех $C_{i}^{\prime}$. Тогда $F_{R^{N}}(V, C)=0$, а $F_{R_{p}}\left(V^{1}, C^{1}\right)=C^{1}$, $F_{R_{p}}\left(V^{2}, C^{2}\right)=C^{2}$.

\section{§ 8. Категории $\widetilde{R}_{1}^{M}$ и $\widetilde{R}_{1, N}^{M}$, разложение морфизмов на элементарные линки, соотношения между линками}

ОПРЕДЕЛЕНИЕ 8.1. 1) Под графом мы будем понимать неориентированный граф, как и в определении 5.1. Подграфы графа определяются естественным образом. Пусть $\mathcal{G}$ - граф и $\mathcal{G}^{\prime}$ - подграф в $\mathcal{G}$, определим дополнение графа $\mathcal{G}^{\prime}$ в $\mathcal{G}$ как граф $\mathcal{G} \backslash \mathcal{G}^{\prime}$, полученный из $\mathcal{G}$ удалением всех вершин и ребер $\mathcal{G}^{\prime}$, а также ребер, соединяющих вершины $\mathcal{G}$ с вершинами $\mathcal{G}^{\prime}$. Деревом, как обычно, мы будем называть связный граф без циклов.

2) Пусть $\mathcal{G}$ - дерево и $\mathcal{G}^{\prime}$ - подграф в $\mathcal{G}$; тогда $\mathcal{G}^{\prime}$ мы будем называть внешним, если $\mathcal{G} \backslash \mathcal{G}^{\prime}-$ дерево.

3) Пусть $\mathcal{G}$ - дерево и $\mathcal{G}^{\prime}-$ подграф в $\mathcal{G}$. Определим замыкание $\mathcal{G}^{\prime}$ в $\mathcal{G}$, обозначаемое $\overline{\left(\mathcal{G}^{\prime}\right)^{\mathcal{G}}}$, как минимальное поддерево в $\mathcal{G}$, содержащее $\mathcal{G}^{\prime}$.

4) Пусть $\mathcal{G}=\sum_{i=1}^{n} \mathcal{G}_{i}$ - объединение конечного числа деревьев, $\mathcal{G}_{i}^{\prime}$ - подграф в $\mathcal{G}_{i}, \mathcal{G}^{\prime}=\sum_{i=1}^{n} \mathcal{G}_{i}^{\prime}$; тогда $\overline{\left(\mathcal{G}^{\prime}\right)^{\mathcal{G}}}$ определяется равенством $\overline{\left(\mathcal{G}^{\prime}\right)^{\mathcal{G}}}=\sum_{i=1}^{n} \overline{\left(\mathcal{G}_{i}^{\prime}\right)^{\mathcal{G}_{i}}}$.

ЗАмечаниЕ 8.2. Пусть $\mathcal{G}$ - дерево и $\mathcal{G}^{\prime}$ - подграф в $\mathcal{G}$. Тогда:

1) если $\mathcal{G}^{\prime}$ - внешнее поддерево $\mathcal{G}$, то существует единственная вершина $a \in$ $\operatorname{Som}(\mathcal{G})$, где $\operatorname{Som}(\mathcal{G})$ - множество вершин $\mathcal{G}$, степень которой в $\mathcal{G}$ на единицу больше ее степени в $\mathcal{G}^{\prime}$ (см. определение 5.1 );

2) из п. 1) несложно вывести, что $\mathcal{G}^{\prime}$ является внешним поддеревом в $\mathcal{G}$, если и только если существуют вершина $a \in \operatorname{Som}(\mathcal{G})$, ассоциированные с ней 
ориентация на $\mathcal{G}$ и отображение $\mathcal{V}: \operatorname{Som}(\mathcal{G}) \rightarrow \mathbb{Z}$ такие, что $\mathcal{V}\left(\operatorname{Som}\left(\mathcal{G}^{\prime}\right)\right) \geqslant 0$, $\mathcal{V}\left(\operatorname{Som}\left(\mathcal{G} \backslash \mathcal{G}^{\prime}\right)\right)<0$

3) из п. 2) следует, что любой внешний подграф содержит вершину степени один в $\mathcal{G}$, если $\mathcal{G}$ не состоит из одной вершины;

4) $\mathcal{G}^{\prime}$ является внешним в $\mathcal{G}$, если и только если каждая связная компонента $\mathcal{G}^{\prime}$ - внешнее поддерево в $\mathcal{G}$.

Teорема 8.3. Пусть $(V, C) \in \mathrm{Ob} R_{1_{-}}, F \subset H \subset G \subset C$ - кривые на $V$, $(G, C-G)=0, H \neq G, \mathcal{G}(G)$ - дерево, кривая $H$ стягиваема в категории $R_{1_{-}}$ и $\mathcal{G}(F)$ - внешний подграф в $\mathcal{G}(G)$. Тогда кривая $F$ стягиваема в категории $R_{1}$.

ДокАЗАтЕЛьСтво. Из теоремы 5.12, замечания 8.2,3), стягиваемости кривой $H$ в категории $R_{1}$ и условия $H \neq G$ следует, что существует неприводимая компонента $E^{0}$ кривой $F$ такая, что $\left(E^{0}, G-E^{0}\right)=1, p_{a}\left(E_{0}\right)=0,\left(E^{0}\right)^{2}<0$. Докажем, что $E^{0}$ содержит не более одной особой точки пары $(V, C)$ типа $0_{n, 1, k}$ (см. определение в 5.2 ), где $k \leqslant 2$. Допустим противное. Пусть $\varphi-$ стягивание кривой $H, \varphi=\varphi_{n} \circ \cdots \circ \varphi_{1}$ - разложение морфизма $\varphi$; тогда из теоремы 5.12 следует, что для любого $i:\left(\varphi_{i} \circ \cdots \circ \varphi_{1}\right)_{*}\left(E^{0}\right)-$ кривая, содержащая более одной особой точки. Тогда из условия $H \neq G$ следует, что кривая $H$ не стягиваема в категории $R_{1_{-}}$. Противоречие.

Поэтому согласно теореме 5.12 кривая $E^{0}$ стягиваема в категории $R_{1}$. Пусть $\sigma_{1}:(V, C) \rightarrow\left(V^{1}, C^{1}\right)$ - ее стягивание, $G^{1}=\left(\sigma_{1}\right)_{*}(G), H^{1}=\left(\sigma_{1}\right)_{*}(H), F^{1}=$ $\left(\sigma_{1}\right)_{*}(F)$. Очевидно, если $F^{1} \neq 0$, то кривая $H^{1}$ стягиваема в категории $R_{1_{-}}$и подграф $\mathcal{G}\left(F^{1}\right)$ является внешним в $\mathcal{G}\left(G^{1}\right)$. Тогда аналогично можно построить стягивание $\sigma_{2}:\left(V^{1}, C^{1}\right) \rightarrow\left(V^{2}, C^{2}\right)$ неприводимой кривой $E^{1} \in F^{1}, \sigma_{2} \in$ Mor $X$. Продолжая этот процесс, мы получим стягивание кривой $F$ в категории $R_{1}$. Теорема доказана.

ЗАмечание 8.4. Пусть $(V, C) \in \mathrm{Ob}^{M} R_{1_{-}}$и кривая $F_{R_{1_{-}}}(V, C)$ непуста и состоит из неприводимых связных компонент, имеющих типы $0_{n_{i}, 0, k_{i}}$ в $(V, C)$, где $k_{i} \leqslant 2$ (см. определение 7.1). Тогда $F_{R_{1}}(V, C)=F_{R_{1_{-}}}(V, C)$. Действительно, достаточно заметить, что для каждой компоненты $F_{i}$ кривой $F_{R_{1}}(V, C)$ легко привести объект $\left(\left(W, C^{W}\right), \varphi, \varphi^{\prime}\right)$ категории $B_{2}\left(R_{1}\right)$ такой, что $\varphi:\left(W, C^{W}\right) \rightarrow$ $(V, C)$ и морфизм $\varphi^{\prime}$ стягивает кривую $\varphi_{\text {соб }}^{-1}\left(F_{i}\right)$. Если $(V, C) \in \mathrm{Ob}^{M} R_{1_{-}}$и кривая $F_{R_{1}}(V, C)$ непуста, то $F_{R_{1}}(V, C)=F_{R_{1_{-}}}(V, C)$.

ОПРЕДЕЛЕНИЕ 8.5. Из теоремы 8.3 и замечания 8.4 следует, что можно определить полные подкатегории $\widetilde{R}_{1}^{M}$ и $\widetilde{R}_{1, N}^{M}$ в категории $\widetilde{R}_{1-}^{M}$ условиями

$$
\begin{gathered}
\text { Ob } \widetilde{R}_{1}^{M}:=\left\{(V, C) \in \mathrm{Ob} \widetilde{R}_{1_{-}}^{M} \mid F_{R_{1}}(V, C) \neq \varnothing\right\}, \\
\text { Ob } \widetilde{R}_{1, N}^{M}:=\left\{(V, C) \in \mathrm{Ob} \widetilde{R}_{1_{-}, N}^{M} \mid F_{R_{1}}(V, C) \neq \varnothing\right\} .
\end{gathered}
$$

СледСтвиЕ 8.6. 1) Пусть $\left(\left(W, C^{W}\right), \varphi, \varphi^{\prime}\right) \in \mathrm{Ob}^{M} B_{2}\left(R_{1_{-}}\right), \varphi:\left(W, C^{W}\right) \rightarrow$ $(V, C), \varphi^{\prime}:\left(W, C^{W}\right) \rightarrow\left(V^{\prime}, C^{\prime}\right), F \subset C-$ кривая на $V$ и $\mathcal{G}(F)$ - обгединение конечного числа деревъев, $F^{\prime}=\varphi_{*}^{\prime} \circ \varphi^{-1}(F),(F, C-F)=0$. Тогда очевидно, чmo

$$
\mathcal{G}\left(\varphi^{-1}(F)\right)=\overline{\mathcal{G}\left(\left(\varphi_{\text {соб }}^{-1}(F)\right)+\mathcal{G}\left(\left(\varphi^{\prime}\right)_{\text {соб }}^{-1}\left(F^{\prime}\right)\right)\right)^{\mathcal{G}(C)}} .
$$

2) Пусть $\gamma \in \operatorname{Mor} \widetilde{R}_{1}^{M}, \gamma:(V, C) \rightarrow\left(V^{\prime}, C^{\prime}\right),\left(\left(W, C^{W}\right), \varphi, \varphi^{\prime}\right) \in L_{R_{1_{-}}}(\gamma), F=$ $F_{R_{1}}(V, C)=\sum_{i=1}^{n} F_{i}$, где $F_{i}$ - неприводимые связные компоненты кривой $F$, 
$F^{W}=\varphi^{-1}(F)=\sum_{i=1}^{n} F_{i}^{W}, F_{i}^{W}=\varphi^{-1}\left(F_{i}\right)=\sum_{j=1}^{m_{i}} F_{i}^{W, j}, F_{i}^{\prime}=\varphi_{*}^{\prime} \circ \varphi^{-1}\left(F_{i}\right)-$ неприводимые связные компоненты кривой $F_{R_{1}}\left(V^{\prime}, C^{\prime}\right)$. Тогда из $\left.n .1\right)$ вытекает, что кривые $F_{i}^{W}$ имеют типь $0_{n_{i}, 0, k_{i}}$, где $k_{i} \leqslant 2$, и что нумерацию на кривой $F^{W}$ можно выбрать так, что

$$
F_{i}^{W, 1}=\varphi_{\text {соб }}^{-1}\left(F_{i}\right), \quad F_{i}^{W, m_{i}}=\left(\varphi^{\prime}\right)_{\text {соб }}^{-1}\left(F_{i}^{\prime}\right) .
$$

ОПРЕДЕлЕНИЕ 8.7. В обозначениях следствия 8.6,2) пусть $\left(\left(W, C^{W}\right), \varphi, \varphi^{\prime}\right) \in$ $L_{R_{1_{-}}}(\gamma)$, где $\gamma \in \operatorname{Mor} \widetilde{R}_{1}^{N}$. Положим

$$
\left(V_{1}^{1}, C_{1}^{1}\right)=(V, C), \quad\left(V_{n}^{m_{n}}, C_{n}^{m_{n}}\right)=\left(V^{\prime}, C^{\prime}\right) .
$$

Рассмотрим морфизмы $\sigma_{i}^{j} \in$ Mor $R_{1}$, где $i \in\{1, \ldots, n\}, j \in\left\{1, \ldots, m_{i}\right\}$. Каждый морфизм $\sigma_{i}^{j}:\left(W, C^{W}\right) \rightarrow\left(V_{i}^{j}, C_{i}^{j}\right)$ является стягиванием следующих компонент кривой $F^{W}: F_{l}^{W, p}$, где $l<i, p<m_{i}, F_{i}^{W, r}$, где $j<r \leqslant m_{i}$ или $j>r$, и $F_{f}^{W, s}$, где $f>i, s>1$. Очевидно, что морфизмы $\sigma_{i}^{j}$ корректно определены и $\left(V_{i}^{j}, C_{i}^{j}\right) \in \mathrm{Ob}^{M} R_{1}$.

Определим морфизмы $\gamma_{i}^{j} \in \operatorname{Mor} \widetilde{R}_{1}^{M}$, где $i \in\{1, \ldots, n\}, j \in\left\{1, \ldots, m_{i}-1\right\}$, условиями $\left(\left(W, C^{W}\right), \sigma_{i}^{j}, \sigma_{i}^{j+1}\right) \in \gamma_{i}^{j}$.

ТЕОРема 8.8. В обозначениях определения 8.7 имеем

$$
\gamma=\gamma_{n}^{m_{n}-1} \circ \cdots \circ \gamma_{n}^{1} \circ \cdots \circ \gamma_{1}^{m_{1}-1} \circ \cdots \circ \gamma_{1}^{1}
$$

ДокАЗАТЕЛЬСтво вытекает из следствия 8.6.

ЗАмечАниЕ 8.9. 1) Морфизмы $\gamma_{i}^{j}$ элементарны в следующем смысле: пусть $\left(\left(Z_{i}^{j}, C^{Z_{i}^{j}}\right), \rho_{i}^{j}, \rho_{i}^{j+1}\right) \in L_{R_{1_{-}}}\left(\gamma_{i}^{j}\right)$ при $j<m_{i}$; тогда $\rho_{i}^{j}, \rho_{i}^{j+1} \in \operatorname{Mor}^{1} R_{1}$.

2) Пусть $\gamma \in \operatorname{Mor} \widetilde{R}_{1, N}^{M}$; тогда из теоремы 6.13 следует, что $\gamma_{i}^{j} \in \operatorname{Mor} \widetilde{R}_{1, N}^{M}$. Если $\varepsilon_{i}^{j}=\widetilde{\mathcal{N}}_{R_{1}}\left(\gamma_{i}^{j}\right)$, то $\varepsilon_{i}^{j} \in$ Mor $\widetilde{R}^{N}$ совпадает с некоторым морфизмом $\varepsilon_{F, p}$, описанным в определении 7.14.

ЗАмечАНИЕ 8.10. Пусть $X$ - одна из категорий $R^{N}, R_{p_{\#}}$ при $4 / 5<p<1$ или $R_{1_{-}}, \gamma \in \operatorname{Mor} \widetilde{X}_{N}^{M}, \gamma:(V, C) \rightarrow\left(V^{\prime}, C^{\prime}\right)$, пара $(V, C)$ стандартна (см. определение 7.13) и кривая $F_{X}(V, C)$ либо состоит из более чем одной связной компоненты, либо имеет численный тип $0_{n, m, k}\left[(a)_{i}\right]$ в $(V, C)$. Тогда из теоремы 8.8 следует, что морфизм $\gamma$ разложим в последовательность морфизмов $\varepsilon_{F_{i}, p_{i}}$, если $X$ является категорией $R^{N}$, и последовательность минимизаций морфизмов $\varepsilon_{F_{i}, p_{i}}$ в противном случае.

Теорема 8.11. В условиях определения 8.7 между элементарными морфизмами $\gamma_{i}^{j}$ категории $\widetilde{R}_{1, N}^{M}$, действующими на одной связной компоненте, отсутствуют нетривиальные соотношения. Морфизмы, действующие на разных компонентах, коммутируют.

ДокАзАтЕльство. Докажем сначала, что между элементарными морфизмами, действующими на одной связной компоненте, отсутствуют нетривиальные соотношения. Тогда можно считать, что кривая $C$ из определения 8.7 состоит из одной связной компоненты. Допустим, что $\gamma_{i}^{1} \circ \cdots \circ \gamma_{i}^{n}-$ соотношение такое, что любое подслово данного слова не является соотношением. Тогда 
если $\left(\left(Z_{i}, C^{Z_{i}}\right), \rho_{i}^{0}, \rho_{i}^{n-1}\right) \in L_{R_{1}}\left(\gamma_{i}^{1} \circ \cdots \circ \gamma_{i}^{n-1}\right)$, то граф, соответствующий кривой $F_{R_{1_{-}}}\left(Z_{i}, C_{i}\right)$, является цепью. Заметим, что если $\left(\left(W_{i}, C_{i}^{W}\right), \varphi_{i}, \varphi_{i}^{\prime}\right)$ - минимальное разрешение морфизма $\gamma_{i}^{1} \circ \cdots \circ \gamma_{i}^{n}$ такое, что $\left(W_{i}, C_{i}^{W}\right)$ доминирует $\left(Z_{i}, C^{Z_{i}}\right)$, то граф, соответствующий кривой $F_{R_{1}}\left(W_{i}, C_{i}^{W}\right)$, является деревом и либо получается добавлением одного звена к цепи, соответствующей кривой $F_{R_{1_{-}}}\left(Z_{i}, C_{i}\right)$, либо совпадает с ней. В первом случае $\gamma_{i}^{1} \circ \cdots \circ \gamma_{i}^{n}$ не может быть соотношением, во втором случае $\gamma_{i}^{1} \circ \cdots \circ \gamma_{i}^{n}$ может быть соотношением тогда и только тогда, когда $n=2$ и $\gamma_{i}^{1}=\left(\gamma_{i}^{2}\right)^{-1}$. Таким образом, первое утверждение доказано. То, что элементарные морфизмы, действующие на разных связных компонентах коммутируют, очевидно. Теорема доказана.

\section{§9. Автоморфизмы квазипроективных поверхностей}

ОПРЕДЕЛЕНИЕ 9.1. 1) Пусть $U$ - квазипроективная поверхность; тогда мы

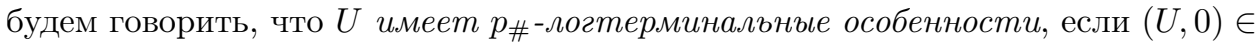
$\mathrm{Ob} R_{\#}^{*}$, где категории $R_{\#}^{*}$ определены в замечании 3.16 . Исходя из результатов $\S 4$ несложно убедиться в том, что если поверхность $U$ имеет $p_{\#}$-логтерминальные особенности, то она имеет также и 1-логтерминальные особенности.

2) Пусть квазипроективная поверхность $U$ имеет 1-логтерминальные особенности. Определим категорию $T^{U}$ компактификаций поверхности $U$ в категории $R_{1_{-}}$условиями:

$$
\begin{gathered}
\operatorname{Ob} T^{U}:=\{(\rho, V, C) \mid \rho: U \hookrightarrow V \text { - открытое вложение, } \\
\left.\quad(V, C) \in \mathrm{Ob} R_{1}=\mathrm{Ob} R_{1_{-}} \text {и } C=V \backslash \rho(U)\right\}, \\
\operatorname{Mor} T^{U}:=\left\{\varphi:(\rho, V, C) \rightarrow\left(\rho^{\prime}, V^{\prime}, C^{\prime}\right) \mid \varphi:(V, C) \rightarrow\left(V^{\prime}, C^{\prime}\right),\right. \\
\left.\varphi \in \operatorname{Mor} R_{1_{-}}, \varphi \circ \rho=\rho^{\prime}\right\} .
\end{gathered}
$$

3) Из выполнения правила Оре в категории $R_{1_{-}}$следует его выполнение в категории $T^{U}$, поэтому корректно определена категория частнъх $\widetilde{T}^{U}$ категории $T^{U}$.

Через $R_{1}^{U}$ мы будем обозначать полную подкатегорию в $R_{1_{-}}$, объектами которой являются пары, компактифицирующие поверхность $U$ в этой категории. Заметим, что категория $R_{1-}^{U}$ получается из категории $T^{U}$ функтором забывания открытого вложения $\rho$. Через $\widetilde{R}_{1_{-}}^{U}$ мы будем обозначать категорию частных категории $R_{1_{-}}^{U}$.

ЗАмечАниЕ 9.2. Пусть поверхность $U$ имеет 1-логтерминальные особенности и $\operatorname{Aut}(U)$ - группа ее бирегулярных автоморфизмов. Тогда, очевидно, существует компактификация $(\rho, V, C) \in \mathrm{Ob} T^{U}$. Поскольку категория $R_{1_{-}}$ является полной подкатегорией в категории $R$, любому бирегулярному автоморфизму $g \in \operatorname{Aut}(U)$, заданному вместе с компактификациями $(\rho, V, C)$, $\left(\rho^{\prime}, V^{\prime}, C^{\prime}\right) \in \mathrm{Ob} T^{U}$ взаимно однозначно соответствует морфизм

$$
\gamma \in \operatorname{Mor} \widetilde{R}_{1_{-}}^{U}, \quad \gamma=\rho^{\prime} \circ g \circ \rho^{-1}:(V, C) \rightarrow\left(V^{\prime}, C^{\prime}\right) .
$$


Опишем теперь классы рассматриваемых компактификаций и поверхностей и возникающие в этих случаях альтернативы. Пусть $U$ имеет 1 -логтерминальные особенности; тогда мы будем рассматривать такие $(\rho, V, C) \in \mathrm{Ob} T^{U}$, что $(V, C) \in \mathrm{Ob}^{M} R_{1_{-}}$и $\mathcal{N}_{R_{1_{-}}}(V, C) \in \mathrm{Ob}^{M} R^{N}$ (очевидно, что такие компактификации всегда существуют). В случае, когда кривая $F_{R_{1}}(V, C)$ имеет тип $0_{n, m, k}$ в $(V, C)$ и может быть приведена к стандартному виду, будут рассматриваться только стандартные компактификации (см. определения 7.1, 5.2 и 7.13). Обозначим через $T_{N}^{U, M}$ полную подкатегорию в $T^{U}$, соответствующую определенному выше классу компактификаций.

Чтобы не вдаваться в излишние подробности, мы ограничимся рассмотрением таких поверхностей $U$, для компактификаций которых выполнено условие $F_{R_{1_{-}}}(V, C)\left(C-F_{R_{1_{-}}}\right)=0$. Тогда согласно результатам из $\S 7$ имеются следующие альтернативы:

1) $F_{R_{1}}(V, C) \neq 0$; тогда $F_{R_{1}}(V, C)$ либо является объединением непересекающихся кривых с нулевым индексом самопересечения, не проходящих через особенности $V$, либо состоит из одной компоненты, проходящей через не более чем две особые точки поверхности $V$ типа $0_{n, m, k}$ в $(V, C)$;

2) $F_{R_{1}}(V, C)=0$, а $F_{R_{1_{-}}}(V, C) \neq 0$; тогда либо $F_{R_{1_{-}}}(V, C)$ имеет тип $1_{n, 0, k}$ в $(V, C)$ и является колесом (см. замечание $5.3,2)$ ), либо $U$ имеет компактификацию такую, что $F_{R_{1}}(V, C)$ состоит из двух компонент $F_{1}, F_{2}$, не проходящих через особенности $V$ и таких, что $F_{1}^{2}=F_{2}^{2}=1,\left(F_{1}, F_{2}\right)=1$;

3) $F_{R_{1_{-}}}(V, C)=0$.

Рассмотрим случай $F_{R_{1}}(V, C) \neq 0$. В определении 8.7 были введены элементарные морфизмы категории $\widetilde{R}_{1, N}^{M}$, на которые можно разложить любой морфизм этой категории. Очевидно, любой морфизм категории $\widetilde{T}_{N}^{U, M}$ также раскладывается на соответствующие этим морфизмам элементарные морфизмы.

ОПРЕДЕЛЕНИЕ 9.3. По аналогии с [9] графом компактификаций $\Delta_{U}$ называется граф, вершинами которого являются классы изоморфных компактификаций поверхности $U$, а ребрами - элементарные морфизмы. Такое определение корректно ввиду того, что элементарные морфизмы переводят изоморфные компактификации в изоморфные. Очевидно, что доказательство существования разложения морфизмов категории $\widetilde{T}_{N}^{U, M}$ на элементарные равносильно доказательству связности графа $\Delta_{U}$.

ОПРЕДЕЛЕНИЕ 9.4. 1) Графом групn $(\Gamma, G)$ называется неориентированный граф Г (здесь неориентированность будет пониматься в смысле [10], т. е. будут рассматриваться направленные ребра, на которых определена операция обращения, равная в квадрате тождественному отображению), каждому ребру и вершине которого сопоставлены некоторые группы, при этом фиксированы вложения групп, соответствующих ребрам, в группы, соответствующие вершинам, инциндентным данным ребрам, иначе говоря, группы на ребрах отождествляются с подгруппами групп на вершинах, которые эти ребра соединяют. Далее через $P_{i}$ мы будем обозначать вершины, через $y_{i}$ - направленные ребра, $\bar{y}_{i}$ - противоположные ребра, а через $G_{P_{i}}, G_{y_{i}}$ - соответствующие группы. Если $g \in G_{y}$, то через $g^{y}$ мы обозначим образ $g$ в группе, соответствующей концу ребра $y$. 
2) Пусть $\Gamma$ - связный граф. Рассмотрим группу $F(\Gamma, G)$, порожденную группами $G_{p_{i}}$ и элементами $y_{i}$, связанными соотношениями

$$
\bar{y}=y^{-1}, \quad y g^{y} y^{-1}=g^{\bar{y}}, \quad g \in G_{y} .
$$

Если $P_{0}$ - некоторая вершина $\Gamma$, то фундаментальной группой графа $(\Gamma, G)$ в $P_{0}$ называется подгруппа $\pi_{1}\left(\Gamma, G, P_{0}\right) \subset F(\Gamma, G)$, порожденная словами вида $g_{0} y_{1} g_{1} \ldots y_{n} g_{n}$, где $g_{i} \in G_{P_{i}}, y_{i}$ - направленное ребро из $P_{i-1}$ в $P_{i}$ и $P_{0}=P_{n}$.

3) Пусть группа $G$ действует на связном графе $\Delta$ и $\Gamma$ - факторграф $\Delta$ по действию $G$. Тогда по этим данным в работе [10] построен граф групn $(\Gamma, G)$, ассоциированный с графом $\Delta$ и действующей на нем группой $G$. Конструкция примерно следующая: строится поднятие вершин и ребер графа $\Gamma$ в $\Delta$, после чего вершинам и ребрам графа $\Gamma$ сопоставляются подгруппы группы $G$, являющиеся стабилизаторами соответствующих вершин и ребер графа $\Delta$. Отображения между группами, соответствующими ребрам и вершинам $\Gamma$, строятся согласно выбору поднятия. То, что в результате действительно будет получен граф групп $(\Gamma, G)$, следует из конструкции.

ТЕорема 9.5 [10, теорема 13]. В обозначениях определения 9.4,3) выполнены следуюшие утверждения:

1) определен сюргективный гомоморфизм $\psi: \pi_{1}\left(\Gamma, G, P_{0}\right) \rightarrow G$, где $P_{0}-$ произвольная вершина Г;

2) граф $\Delta$ является деревом тогда и толъко тогда, когда $\psi$ - изоморфизм.

УТВЕРЖДЕНИЕ 9.6. В предыдущих обозначениях ядро гомоморфизма $\psi$ изоморфно фундаментальной группе графа $\Delta$.

Рассмотрим граф компактификаций $\Delta_{U}$ и действующую на нем группу $\operatorname{Aut}(U)$. Условием $g(\rho)=g \circ \rho$ определено действие группы $\operatorname{Aut}(U)$ на этом графе. Легко проверить, что факторграф $\Gamma_{U}$ графа $\Delta_{U}$ по данному действию изоморфен графу пар, компактифицирующих поверхность $U$ в категории $R_{1, N}^{M}$, вершинами которого являются классы изоморфных пар, а ребрами - элементарные морфизмы категории $\widetilde{R}_{1, N}^{M}$.

Тогда, если $F_{R_{1}}(V, C)$ состоит из одной неприводимой компоненты, между элементарными морфизмами отсутствуют нетривиальные соотношения. Поэтому граф $\Delta_{U}$ не имеет циклов и, следовательно, является деревом. Тогда согласно теореме 9.5 группа $\operatorname{Aut}(U)$ изоморфна фундаментальной группе графа групп $\left(\Gamma_{U}, \operatorname{Aut}(U)\right)$, построенного по факторграфу графа $\Delta_{U}$ по действию $\operatorname{Aut}(U)$ в соответствии с определением 9.4. Для решения поставленной задачи остается вычислить стабилизаторы вершин и ребер $\Delta_{U}$. Легко проверить, что стабилизаторы вершин графа $\Delta_{U}$ изоморфны группам бирегулярных автоморфизмов соответствующих этим вершинам стандартных пар, стабилизаторы ребер также легко определяются по элементарным морфизмам. Таким образом, мы получили полное описание группы $\operatorname{Aut}(U)$ для данного случая.

В случае, когда $F_{R_{1}}(V, C)$ состоит из нескольких неприводимых компонент, элементарные морфизмы, соответствующие различным неприводимым компонентам, коммутируют. Поэтому граф $\Delta_{U}$ имеет циклы и не является деревом, ввиду чего мы можем дать полное описание группы $\operatorname{Aut}(U)$ в соответствии с утверждением 9.6 .

Рассмотрим случай, когда $F_{R_{1}}(V, C)=0$, а $F_{R_{1_{-}}}(V, C) \neq 0$. Если $F_{R_{1_{-}}}(V, C)$ имеет тип $1_{n, 0, k}$ в $(V, C)$, т. е. является колесом, нами не получено разложение 
морфизмов категории $\widetilde{R}_{1_{-}, N}^{M}$ на элементарные, и вряд ли оно могло бы оказаться полезным для описания группы $\operatorname{Aut}(U)$, хотя легко доказать, что связная компонента единицы этой группы совпадает со связной компонентой единицы группы бирегулярных автоморфизмов любой компактифицирующей ее пары (это следует из утверждения 7.8).

В случае, когда $U$ имеет компактификацию такую, что $F_{R_{1}}(V, C)$ состоит из двух компонент $F_{1}, F_{2}$, не проходящих через особенности $\bar{V}$ и таких, что $F_{1}^{2}=F_{2}^{2}=1,\left(F_{1}, F_{2}\right)=1$, разложение на линки легко может быть построено (хотя мы этого и не делаем) методами из [3], но оно не даст полного описания группы $\operatorname{Aut}(U)$, поскольку между линками будут нетривиальные соотношения, которые нам на данный момент не известны.

Рассмотрим случай $F_{R_{1_{-}}}(V, C)=0$. Легко видеть, что в данной ситуации $(V, C)$ является правожестким объектом категории $R_{1_{-}}$, поэтому $\operatorname{Aut}(U)=$ $\operatorname{Aut}(V, C)$ (см. определение 2.3 и замечание 2.18 ).

\section{Список литературы}

1. A. Corti, "Factoring birational maps of threefolds after Sarkisov", J. Algebraic Geom., 4:2 (1995), 223-254.

2. В.А.Исковских, "Факторизация бирациональных отображений рациональных поверхностей с точки зрения теории Мори", УМH, 51:4 (1996), 3-72; англ. пер.: V.A. Iskovskikh, "Factorization of birational maps of rational surfaces from the viewpoint of Mori theory", Russian Math. Surveys, 51:4 (1996), 585-652.

3. A. Bruno, K. Matsuki, "Log Sarkisov program", Internat. J. Math., 8:4 (1997), 451-494.

4. М.Х. Гизатуллин, "Квазиоднородные аффинные поверхности", Изв. АН CCCP. Сер. матем., 35:5 (1971), 1047-1071; англ. пер.: M. H. Gizatullin, "Quasihomogeneous affine surfaces", Math. USSR-Izv., 5:5 (1971), 1057-1081.

5. М. Х. Гизатуллин, "Инварианты неполных алгебраических поверхностей, получаемые с помощью пополнений”, Изв. АН СССР. Сер. матем., 35:3 (1971), 485-497; англ. пер.: М. Н. Gizatullin, "Invariants of incomplete algebraic surfaces obtained by completions", Math. USSR-Izv., 5:3 (1971), 503-515.

6. М.Х. Гизатуллин, "Аффинные поверхности, квазиоднородные относительно алгебраической группы”, Изв. АН СССР. Сер. матем., 35:4 (1971), 738-753; англ. пер.: M.H. Gizatullin, "Affine surfaces which are quasihomogeneous with respect to an algebraic group", Math. USSR-Izv., 5:4 (1971), 754-769.

7. М.Х. Гизатуллин, "Об аффинных поверхностях, пополняемых неособой рациональной кривой”, Изв. АН СССР. Сер. матем., 34:4 (1970), 778-802; англ. пер.: M. H. Gizatullin, "On affine surfaces that can be completed by a nonsingular rational curve", Math. USSR-Izv., 4:4 (1970), 787-810.

8. М. Х. Гизатуллин, В. И. Данилов, "Автоморфизмы аффинных поверхностей. I", Изв. АН СССР. Сер. матем., 39:3 (1975), 523-565; англ. пер.: М. H. Gizatullin, V.I. Danilov, "Automorphisms of affine surfaces. I", Math. USSR-Izv., 9:3 (1975), 493-534.

9. М.Х. Гизатуллин, В. И. Данилов, “Автоморфизмы аффинных поверхностей. II", Изв. АН СССР. Сер. матем., 41:1 (1977), 54-103; англ. пер.: М. H. Gizatullin, V.I. Danilov, "Automorphisms of affine surfaces. II", Math. USSR-Izv., 11:1 (1977), $51-98$. 
10. Ж.П. Серр, “Деревья, амальгамы и $\mathrm{SL}_{2}$ ”, Математика, 18:1 (1974), 3-51.

11. A. Dubouloz, S. Lamy, Variations on log Sarkisov program for surfaces, arXiv: abs/0802.2441.

12. K. Matsuki, Introduction to the Mori program, Universitext, Springer-Verlag, New York, 2002.

13. Yu. Kawamata, K. Matsuda, K. Matsuki, "Introduction to the minimal model problem", Algebraic geometry (Sendai, 1985), Adv. Stud. Pure Math., 10, North-Holland, Amsterdam, 1987, 283-360.

Ю. М. Полякова (Yu. M. РоLyакоva)

Поступило в редакцию

Институт проблем передачи информации

18.06.2007

им. А. А. Харкевича РАН, г. Москва

18.08.2009

E-mail: wainzwei@iitp.ru 